\title{
WestVirginiaUniversity
}

THE RESEARCH REPOSITORY @ WVU

Graduate Theses, Dissertations, and Problem Reports

2012

\section{Oxygen Transport Kinetics in Solid Oxide Fuel Cell Cathode}

Yihong Li

West Virginia University

Follow this and additional works at: https://researchrepository.wvu.edu/etd

\section{Recommended Citation}

Li, Yihong, "Oxygen Transport Kinetics in Solid Oxide Fuel Cell Cathode" (2012). Graduate Theses,

Dissertations, and Problem Reports. 4887.

https://researchrepository.wvu.edu/etd/4887

This Dissertation is protected by copyright and/or related rights. It has been brought to you by the The Research Repository @ WVU with permission from the rights-holder(s). You are free to use this Dissertation in any way that is permitted by the copyright and related rights legislation that applies to your use. For other uses you must obtain permission from the rights-holder(s) directly, unless additional rights are indicated by a Creative Commons license in the record and/ or on the work itself. This Dissertation has been accepted for inclusion in WVU Graduate Theses, Dissertations, and Problem Reports collection by an authorized administrator of The Research Repository @ WVU.

For more information, please contact researchrepository@mail.wvu.edu. 


\title{
Oxygen Transport Kinetics in Solid Oxide Fuel Cell Cathode
}

\author{
Yihong Li
}

Dissertation submitted to

Benjamin M. Statler College of Engineering and Mineral Resources

at West Virginia University

in partial fulfillment of the requirements

for the degree of

\author{
Doctor of Philosophy \\ In \\ Mechanical Engineering \\ Xingbo Liu, Ph.D., Chair \\ Randall S. Gemmen, Ph.D. \\ Kirk R. Gerdes, Ph.D. \\ Edward M. Sabolsky, Ph.D. \\ Nick Wu, Ph. D. \\ Darran R. Cairns, Ph. D.
}

Department of Mechanical and Aerospace Engineering

Morgantown, West Virginia

2012

Keywords: Solid oxide fuel cell; oxygen transport kinetics; cathode electrode process 


\section{Abstract \\ Oxygen Transport Kinetics in Solid Oxide Fuel Cell Cathode}

\section{Yihong Li}

Solid oxide fuel cells (SOFCs) are energy conversion devices that produce electricity by electrochemically combining a fuel and an oxidant across an ionic conducting oxide electrolyte. As it is regarded as the most efficient and versatile power generation system, SOFCs have attracted more substantial interest in recent years. Oxygen reduction at the cathode is considered as the main rate limiting factor to the performance of the whole system. In this work, experimental study of oxygen transport in single phase and infiltrated cathode materials using electrical conductivity relaxation $(E C R)$ technique are combined with physical modeling to benefit SOFCs cathode improvement.

The conductivity relaxation technique involves measurement of time variation of the electrical conductivity of a sample after a stepwise change in the ambient oxygen partial pressure. Oxygen surface exchange $(k)$ and bulk diffusion coefficients $(D)$ can be obtained based on the correlation between a mean conductivity and the corresponding mean non-stoichiometry. Although the ECR technique has been widely used in various applications, reliability and accuracy of fitted results have been rarely discussed. Indeed, non-unique local fitting error minimums exist when fitting a single relaxation data set. Enhanced accuracy of $D$ and $k$ are obtained by fitting two sets of data and plotting the error intersection.

Oxygen surface exchange and bulk diffusion coefficients of the widely used cathode material $\mathrm{La}_{0.6} \mathrm{Sr}_{0.4} \mathrm{Co}_{0.2} \mathrm{Fe}_{0.8} \mathrm{O}_{3-\delta}$ (LSCF) were obtained by applying the improved fitting method. The results indicated that the oxygen surface exchange coefficient depends on the final oxygen partial pressure following the $P_{O_{2}}^{1 / 2}$ law. On the other hand, the oxygen bulk diffusion coefficient was considered to be influenced by the oxygen vacancy concentration and the ordering degree.

Electrical conductivity relaxation was further developed to investigate infiltrated cathode materials in this work. $\mathrm{Ce}_{0.8} \mathrm{Sm}_{0.2} \mathrm{O}_{1.9}$ (SDC) and $\mathrm{La}_{0.6} \mathrm{Sr}_{0.4} \mathrm{CoO}_{3-\delta}$ (LSC) were chosen as the infiltrated materials. The oxygen exchange coefficient at the infiltrate/cathode backbone interface was deduced from the testing results. Both of the two infiltrated materials promoted the oxygen transport rate in LSCF. Under high oxygen partial pressure, the SDC spin coated LSCF sample showed a greater improvement than the LSC spin coated sample.

In addition, a model was built up to understand SOFCs infiltrated cathode. Infiltrate/cathode backbone interface and the corresponding 3PB region distinguished infiltrated SOFCs cathode from single phase cathode. Simulation results are more plausible by including the experimentally obtained oxygen interface exchange coefficient. Over-potential effects and infiltrated material optimization were included in the discussion. 


\section{Acknowledgement}

I would like to gratefully and sincerely thank Dr. Xingbo Liu for his guidance, understanding, patience, and most importantly, his friendship during my graduate studies at West Virginia University. I also want to thank Dr. Kirk Gerdes (National Energy Technology Laboratory, Morgantown, WV) for the discussions that helped me sort out the technical details of my work. And I am deeply grateful to him for encouraging the use of correct grammar and consistent notation in my writings and for carefully reading and commenting on this manuscript.

I also want to appreciate Dr. Randall Gemmen (National Energy Technology Laboratory, Morgantown, WV) and my lab mate Dr. Mingyang Gong's help on the infiltrated cathode model part. Thanks also to Dr. Harvey Diamond (Department of Mathematics, West Virginia University, Morgantown, WV) for his help on electrical conductivity relaxation data analysis. My research was based on electrical conductivity relaxation system. Thus I gladly express my gratitude to the people who help me build up the system---Richard Pineault, Richard Addis and David Ruel in National Energy Technology Laboratory. I also want to acknowledge my appreciation of Dr. Teruhisa Horita's (National Institute of Advanced Industrial Science and Technology, Tsukuba, Japan) help on isotope testing for oxygen transport behavior. And I want to thanks for all my doctoral committee members for their valuable suggestions on my research work.

I also thank all my lab mates for their support and care which helped me overcome setbacks and stay focused on my graduate study. At last, I sincerely appreciate all my family members for their love, concern, support and encouragement. 


\section{CONTENTS}

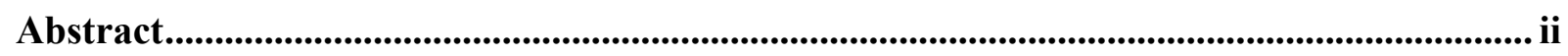

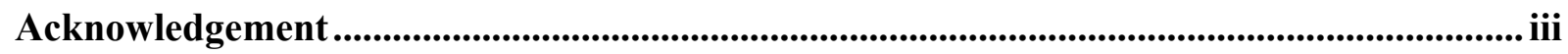

List of Figures.......................................................................................................................................... vii

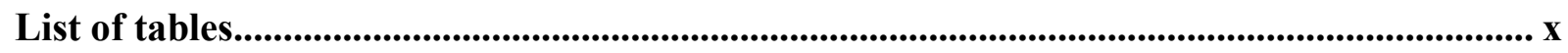

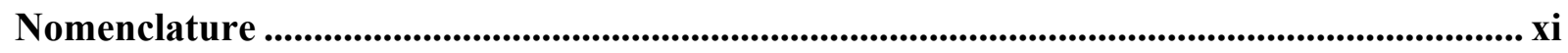

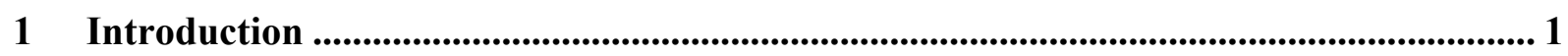

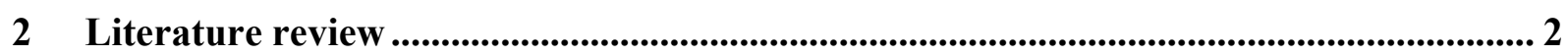

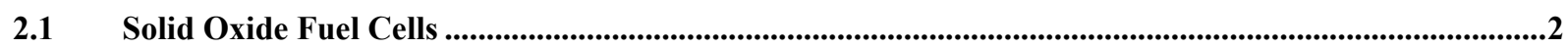

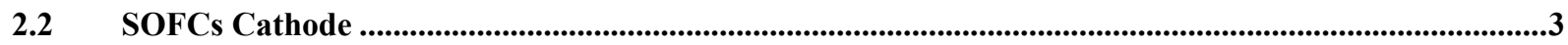

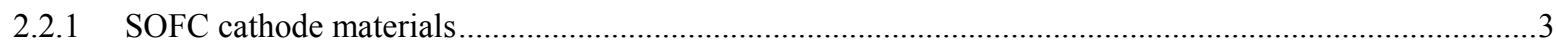

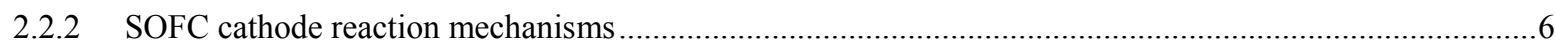

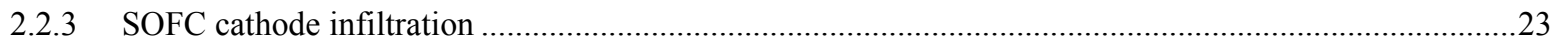

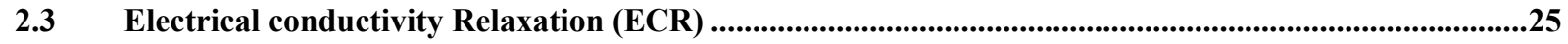

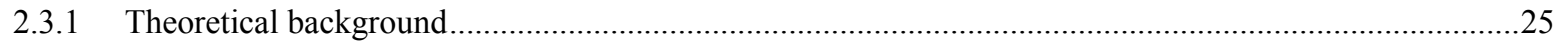

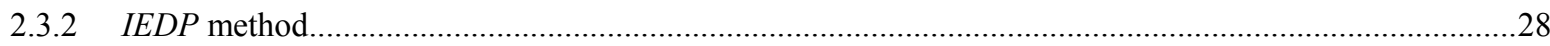

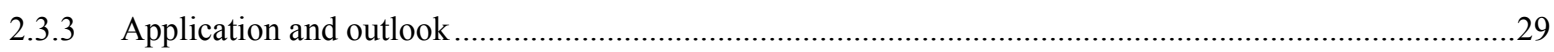

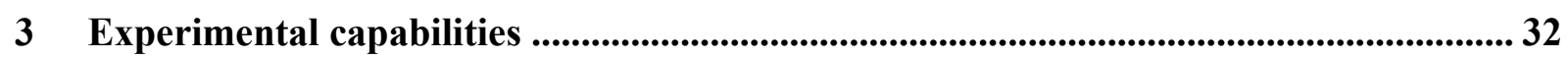

3.1 Electrical conductivity relaxation testing system .............................................................................32

3.2 Manufacture equipments for ECR samples and SOFC cathodes ..........................................................33

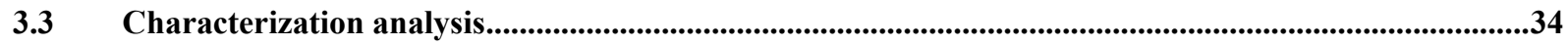

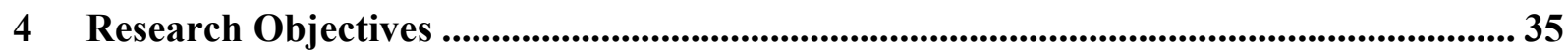

5 Data analysis method for relaxation experiments ............................................................... 36 


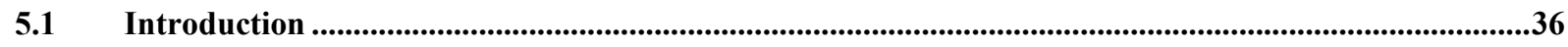

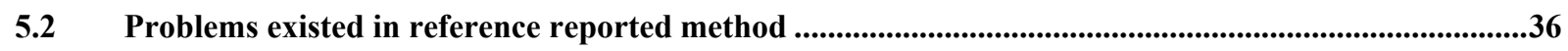

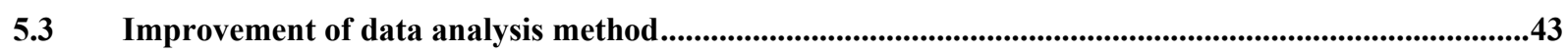

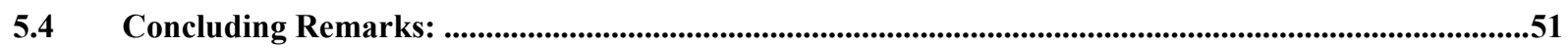

6 Chemical diffusion and oxygen surface exchange of $\mathrm{La}_{0.6} \mathrm{Sr}_{0.4} \mathrm{Co}_{0.2} \mathrm{Fe}_{0.8} \mathrm{O}_{3-\delta}$ studied with electrical conductivity relaxation .........................................................................................5 53

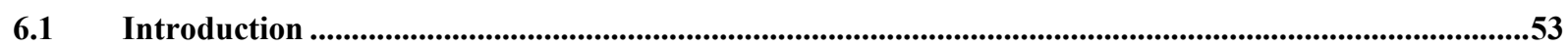

6.2 LSCF pellet preparation and characterization ...........................................................................................53

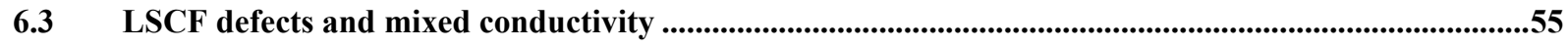

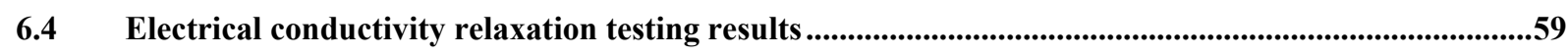

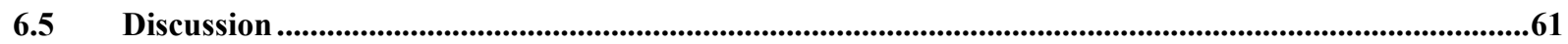

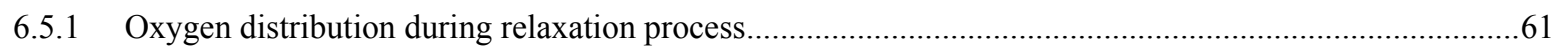

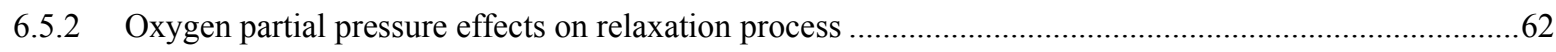

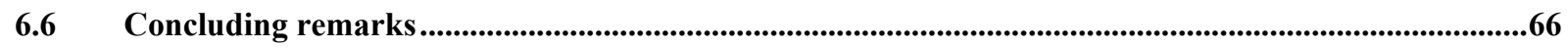

7 Oxygen transport behavior investigation for infiltrated SOFC cathode.......................... 68

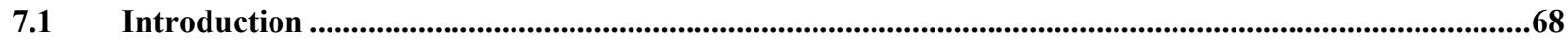

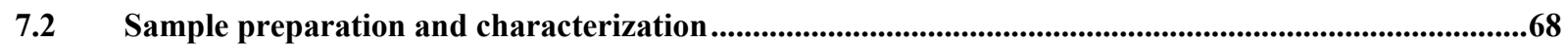

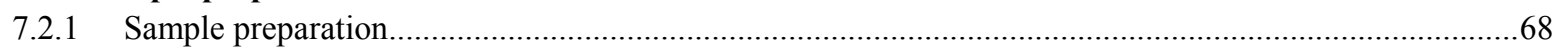

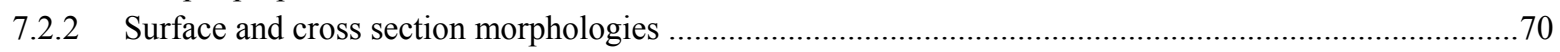

Oxygen transport behavior in infiltrated materials .................................................................................73

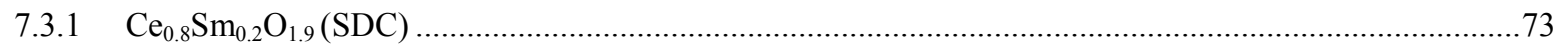

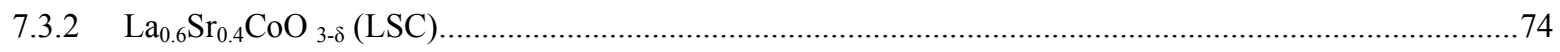

7.4 Oxygen transport behavior at infiltrated/backbone material interface.................................................78

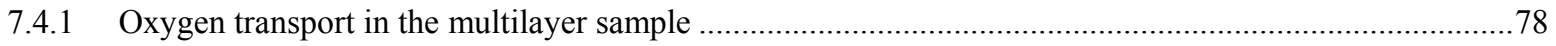

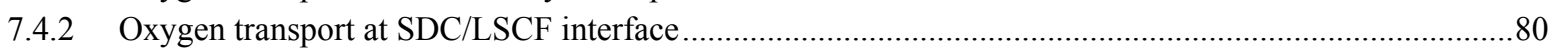

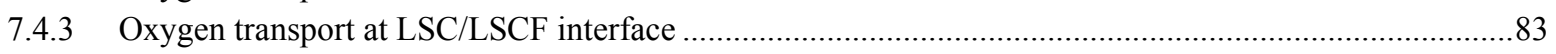

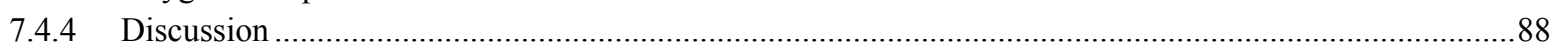

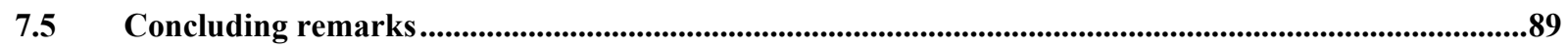

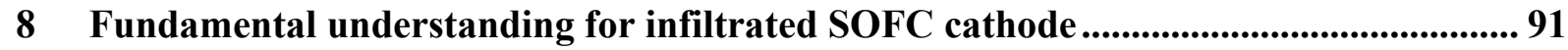




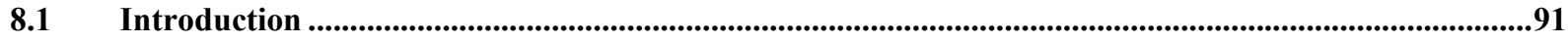

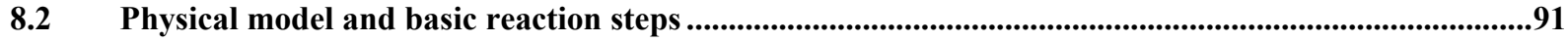

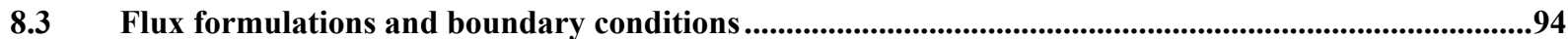

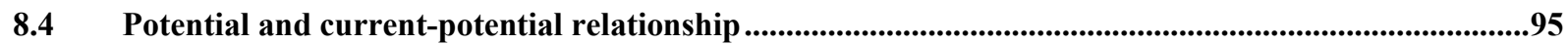

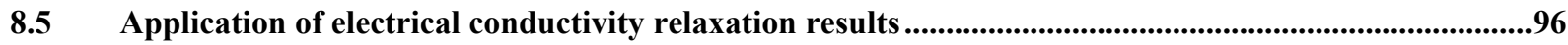

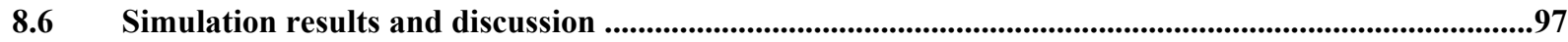

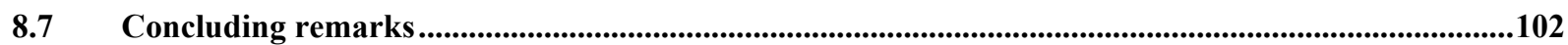

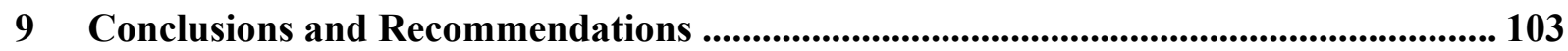

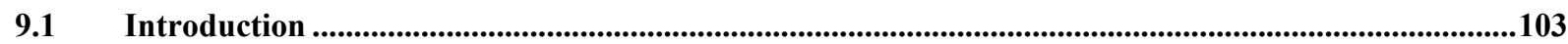

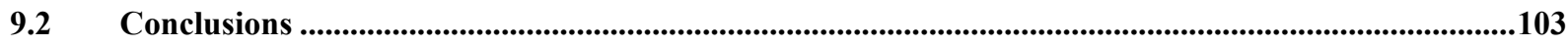

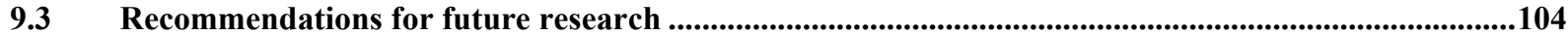

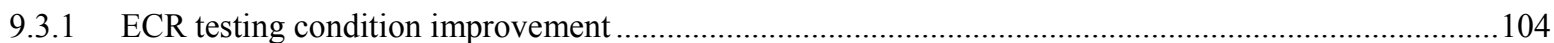

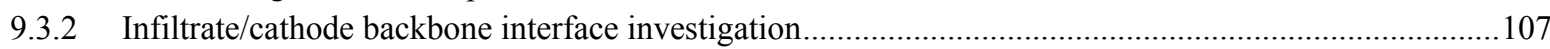

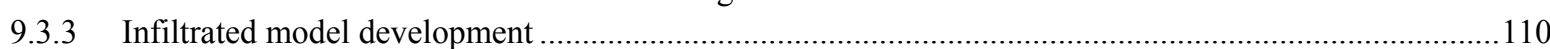




\section{List of Figures}

Cross-sectional schematic of the physical structure and chemical reactions occurring at porous SOFCs cathode

$2.9 \quad$ Sketches of electron and ion transfer reactions at

(a) metal/liquid electrolyte and (b) mixed conducting electrode (MCE)/solid electrolyte interfaces

2.10 An equivalent circuit of the MIEC electrode and the sketch of the oxygen reduction process

$2.11 \quad$ Sketch of 2-D model

(a) patterned electrode array (b) symmetric 2-D cross-sectional model domain

3.1 Electrical conductivity relaxation testing system

3.2 Schematic representation of the conductivity relaxation set-up

5.1 Depiction of fit quality between two methods for determining the starting relaxation time

(a) treating $t_{0}$ as an empirical parameter; and (b) determining $t_{0}$ from reactor parameters and an ideally stirred tank model

(a) Error map for wide range of $\mathrm{k}$ and D; (b) Error map with adjusted scale showing error tolerance band of 5\%
(a) $\mathrm{D}=5 \times 10^{-6} \mathrm{~cm}^{2} / \mathrm{s}, \mathrm{b}_{1}=0.8603$
(b) $\mathrm{D}=3 \times 10^{-5} \mathrm{~cm}^{2} / \mathrm{s}, \mathrm{b}_{1}=0.6000$ 
(a) Error map for wide range of $\mathrm{k}$ and D; (b) Error map with adjusted scale showing error tolerance band of 5\% time

5.7 Error color map for ECR fitting $\left(\mathrm{L}_{1}=1, \mathrm{~L}_{2}=10\right)$

(a) Error map for wide range of $\mathrm{k}$ and $\mathrm{D}$;

(b) Error map with adjusted scale showing error tolerance band of $5 \%$

5.8 Error color map for fitting two relaxation data sets $\left(\mathrm{L}_{1}=1, \mathrm{~L}_{2}=0.1\right)$

(a) Error map for wide range of $\mathrm{k}$ and $\mathrm{D}$;

(b) Error map with adjusted scale showing error tolerance band of $5 \%$

5.9 Error color map for fitting two noisy relaxation data sets $\left(\mathrm{L}_{1}=1\right.$, $\mathrm{L}_{2}=3$ )

(a) Error map for wide range of $\mathrm{k}$ and $\mathrm{D}$;

(b) Error map with adjusted scale showing error tolerance band of $5 \%$

5.10 Error color map for fitting three relaxation data sets $\left(\mathrm{L}_{1}=1, \mathrm{~L}_{2}=0.6\right.$, $\mathrm{L}_{3}=3$ )

(a) Error map for wide range of $\mathrm{k}$ and $\mathrm{D}$;

(b) Error map with adjusted scale showing error tolerance band of $5 \%$

6.1 Surface morphologies and surface chemical composition of sintered LSCF pellet

(a) Before polishing (b) After polishing

XRD patterns for commercial LSCF powder and sintered LSCF pellet

6.3 Schematic representation of concentration of oxygen vacancy

and electronic defects in LSCF as a function of oxygen partial pressure

6.4 Oxygen non-stoichiometry as a function of

oxygen partial pressure for $\mathrm{La}_{0.6} \mathrm{Sr}_{0.4} \mathrm{Co}_{0.2} \mathrm{Fe}_{0.8} \mathrm{O}_{3-\delta}$

6.5 Conductivity of $\mathrm{La}_{0.6} \mathrm{Sr}_{0.4} \mathrm{Co}_{0.2} \mathrm{Fe}_{0.8} \mathrm{O}_{3 \pm \delta}$ at $800^{\circ} \mathrm{C}$ as a function of oxygen partial pressure

6.6 Fitting error color maps for LSCF under different oxygen partial pressure

6.7 Oxygen distributions with different rate-limited steps

6.8 Oxygen surface exchange coefficient for $\mathrm{La}_{0.6} \mathrm{Sr}_{0.4} \mathrm{Co}_{0.2} \mathrm{Fe}_{0.8} \mathrm{O}_{3 \pm \delta}$ at $800^{\circ} \mathrm{C}$ as a function of oxygen partial pressure obtained from conductivity relaxation

6.9 Oxygen bulk diffusion coefficient for $\mathrm{La}_{0.6} \mathrm{Sr}_{0.4} \mathrm{Co}_{0.2} \mathrm{Fe}_{0.8} \mathrm{O}_{3 \pm \delta}$ at $800^{\circ} \mathrm{C}$ as a function of oxygen partial pressure obtained from conductivity relaxation 
7.1 Surface morphologies and surface chemical composition of 70 LSC/LSCF pellet (co-sintered at $1200^{\circ} \mathrm{C}$ )

7.2 Surface and cross section morphologies of spin coated SDC 71 sample

$\begin{array}{llll}\text { 7.3 Surface and cross section morphologies of spin coated LSC } & 72\end{array}$ sample

7.4 Total conductivity dependence on oxygen partial pressure of SDC $\quad 74$

7.5 Surface morphologies and surface chemical composition of 75 sintered LSC pellet

(a) Before polishing (b) After polishing

7.6 Fitting error color maps for LSC under different oxygen partial pressure

7.7 Oxygen transport kinetic parameters for $\mathrm{La}_{0.6} \mathrm{Sr}_{0.4} \mathrm{CoO}_{3-\delta}$ at $800^{\circ} \mathrm{C}$ as a function of oxygen partial pressure obtained from conductivity relaxation

7.8 Sketch for the multilayer sample

Fitting results for SDC/LSCF/SDC multilayer sample

Fitting results for LSC/LSCF/LSC multilayer sample

7.11 Surface exchange coefficients of single phase LSCF and infiltrated cathodes

8.2 Oxygen concentration distributions in the cathode $(\eta=-0.2 \mathrm{~V})$

$r_{3,0}\left(\mathrm{~mol} / \mathrm{cm}^{2} \cdot \mathrm{s}\right)$ value is: (a) $1 \times 10^{-8}$ (b) $2 \times 10^{-8}$ (c) $3 \times 10^{-8}$ (d) $4 \times 10^{-8}$ (e) $5 \times 10^{-8}$

8.3 Oxygen interface exchange rate variation with the cathode thickness $(\eta=-0.2 \mathrm{~V})$

$r_{3,0}\left(\mathrm{~mol} / \mathrm{cm}^{2} \cdot \mathrm{s}\right)$ value is: (a) $1 \times 10^{-8}$ (b) $2 \times 10^{-8}$ (c) $3 \times 10^{-8}$ (d) $4 \times 10^{-8}$ (e) $5 \times 10^{-8}$

8.4 Oxygen vacancy concentration distributions in the cathode $\left(r_{3,0}=3 \times 10^{-8} \mathrm{~mol} / \mathrm{cm}^{2} \cdot \mathrm{s}\right)$

Over-potential: (a) $-0.1 \mathrm{~V}$ (b) $-0.2 \mathrm{~V}$ (c) $-0.3 \mathrm{~V}$ (d) $-0.4 \mathrm{~V}$ (e) $0.05 \mathrm{~V}$ (f) $0.1 \mathrm{~V}$

8.5 Logarithmic $2 \mathrm{~PB}$ current density versus over-potential with $r_{3,0}=3 \times 10^{-8} \mathrm{~mol} / \mathrm{cm}^{2} \cdot \mathrm{s}$

9.1 Electrical conductivity of LSM as a function of oxygen partial pressure at different temperature

9.2 Depth profile of ${ }^{18} \mathrm{O}$ in $\mathrm{La}_{0.6} \mathrm{Sr}_{0.4} \mathrm{Co}_{0.2} \mathrm{Fe}_{0.8} \mathrm{O}_{3-\delta}$ at $800^{\circ} \mathrm{C}$

9.3 Self-surface exchange and bulk diffusivity of $\mathrm{La}_{0.6} \mathrm{Sr}_{0.4} \mathrm{Co}_{0.2} \mathrm{Fe}_{0.8} \mathrm{O}_{3-\delta}$ 


\section{List of tables}

5.1 Parameter values assumed to generate conductivity relaxation data for cases of mixed control $\left(\mathrm{L}_{1}=1\right)$ and bulk diffusion control $\left(\mathrm{L}_{2}=10\right)$

Parameters values of generated conductivity relaxation data $\left(\mathrm{L}_{1}=1, \mathrm{~L}_{2}=0.1\right)$

5.4 Parameters values of generated conductivity relaxation data

7.1 Basic oxygen transport kinetic parameters for simulation 79

7.2 Total oxygen surface exchange coefficient of spin coated SDC sample

7.3 Total oxygen surface exchange coefficient of spin coated LSC

7.4 Oxygen interface exchange coefficient of spin coated LSC

8.1 Parameter values applied for simulation 


\section{Nomenclature}

\section{Roman letters}

\begin{tabular}{ll}
$\mathrm{A}$ & Area \\
$a$ & Half thickness of the ECR pellet sample \\
$b$ & Unit-less parameter for solving Fick's second \\
$\mathrm{C}$ & law \\
$c$ & Concentration \\
$\mathrm{D}$ & Thickness of the spin coated layer \\
$\mathrm{D}^{*}$ & Oxygen diffusion coefficient \\
$\mathrm{E}$ & Self-diffusion coefficient \\
$\mathrm{F}$ & Potential \\
& Fitting error vector \\
$f$ & Faraday constant \\
& Weight average fitting error for two ECR data \\
$\mathrm{J}$ & sets \\
$\mathrm{K}$ & Thermal factor f=F/RT \\
$k$ & Flux \\
$k^{*}$ & Reaction equilibrium constant \\
$k_{n}$ & Oxygen exchange coefficient \\
$\mathrm{L}$ & Self-exchange coefficient \\
& Reaction rate constant \\
$l_{c}$ & Unit-less parameter for solving Fick's second \\
$n$ & law \\
$\mathrm{P}$ & Characteristic length for SOFCs cathode \\
$\mathrm{P}_{\mathrm{L}}$ & Natural number \\
$\mathrm{R}$ & Pressure \\
$r$ & Intersection counts \\
$\mathrm{S}_{\mathrm{V}}$ & Gas Constant \\
$\mathrm{T}$ & Reaction rate \\
$t$ & Specific surface area \\
$\mathrm{V}$ & Temperature \\
$\mathrm{V}_{\mathrm{V}}$ & ECR data length \\
& Time \\
& Reactor volume \\
& Volume fraction porosity \\
& \\
\hline & \\
&
\end{tabular}

Greek letters

$\sigma$

$\tau_{\mathrm{f}}$

$\phi_{v, t o t}$

$\pi$
Area

Half thickness of the ECR pellet sample Unit-less parameter for solving Fick's second law

Concentration

Thickness of the spin coated layer

Oxygen diffusion coefficient

Self-diffusion coefficient

Potential

Fitting error vector

Faraday constant

sets

Thermal factor $\mathrm{f}=\mathrm{F} / \mathrm{RT}$

Self-exchange coefficient

Reaction rate constant

Unit-less parameter for solving Fick's second

law

Natural number

Pressure

Intersection counts

Gas Constant

Reaction rate

Specific surface area

Temperature

ECR data length

Time

Volume fraction porosity

Conductivity

Reactor flush time

Total gas flow rate

$\mathrm{Pi}$ 
Superscripts

\section{Subscripts}

$r$

STP

0

$o$

$S$

V

I

$e q$

Operational symbols

[ ]

$\infty$
Surface oxygen adsorption site density Thermodynamic factor

Degree of coverage of adsorbed oxygen Over-potential

Symmetry factor

Reverse reaction

Reactor

Reduction

Standard temperature and pressure

Initial condition

Oxidation

Surface

Oxygen vacancy

Infiltrated material

Equilibrium

Concentration

Unit

be in proportion to 


\section{Introduction}

The high operating temperature of SOFC relative to other types of fuel cells makes them ideal for combined cycle FC/turbine applications ${ }^{1}$. On the other hand, compared to other kinds of fuel cells, the fuel flexibility of SOFCs offers the possibility for direct utilization of hydrocarbons and other renewable fuels ${ }^{2}$.

It has been well accepted that oxygen reduction occurring at the cathode is the main rate factor limiting the performance of the whole SOFC system ${ }^{3,4}$. Therefore, modeling and simulation techniques have been used to improve understanding of the reaction mechanisms and kinetics of cathode process in SOFC. There are two main understandings of the oxygen reduction process. Some researchers considered that the reduction reaction is a pure chemical reaction while others believed that it's an electrochemical reaction.

Although significant advances have been made in understanding of solid oxide fuel cell cathodes, the oxygen reduction mechanisms remain unknown. The simulation results of present models remain weak since accurate, experimentally obtained oxygen transport kinetic parameters were not used in the simulation. On the other hand, the accuracy of solid state electrochemistry analysis techniques limited the verification work.

In the proposed research, electrical conductivity relaxation (ECR) will be applied to characterize cathode materials for common and advanced SOFC. To obtain more reliable kinetic parameters, the diffusion model and data fitting method of ECR was refined, and detailed discussion is provided. Further, the oxygen reduction mechanism for infiltrated SOFC cathode will be investigated, and the developed ECR diffusion model will be utilized to propose optimized cathode infiltrate materials. 


\section{Literature review}

\subsection{Solid Oxide Fuel Cells}

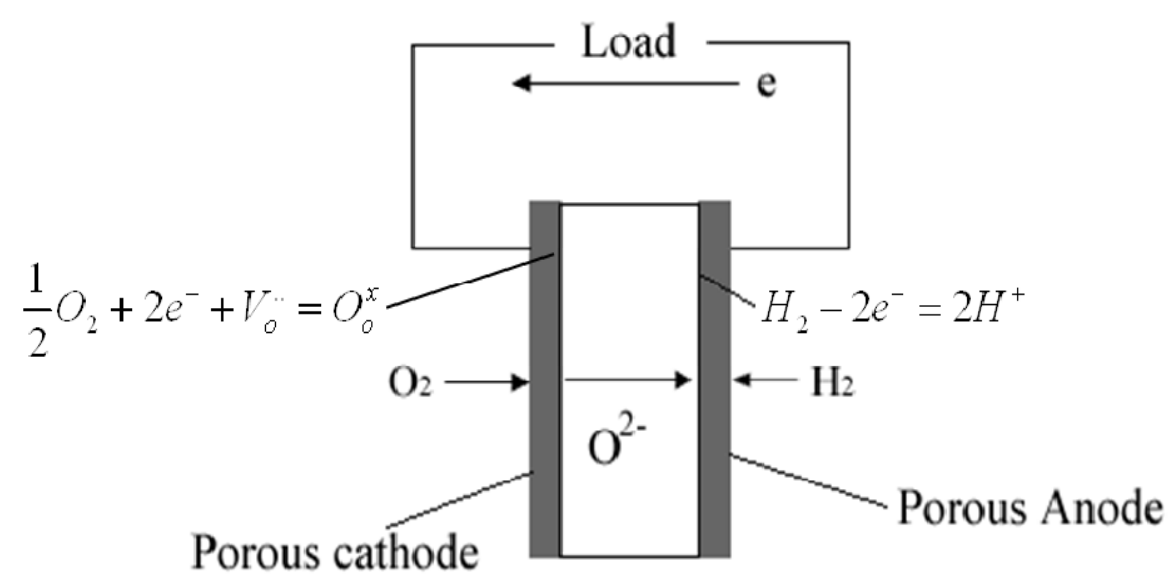

Figure 2.1 Sketch of a single Solid Oxide Fuel Cell

Solid oxide fuel cells (SOFCs) are energy conversion devices that produce electricity by electrochemically combining a fuel and an oxidant across an ionically conductive oxide electrolyte $^{5}$. Figure 2.1 illustrates the basic operation mechanism of the solid oxide fuel cell. The cell is composed of two porous electrodes and an ionically conductive ceramic electrolyte. At present, the typical materials for SOFCs are oxide ion conducting yttria-stablized zirconia (YSZ) for the electrolyte, perovskites such as strontium-doped lanthanum manganese (LSM) for the cathode and nickel/YSZ for the anode ${ }^{6}$. A general reaction mechanism of SOFC is shown in Figure 2.1. Oxygen will be reduced to $\mathrm{O}^{2-}$ at the porous cathode and then be transported through the electrolyte to the anode. The reduced $\mathrm{O}^{2-}$ will react with $\mathrm{H}_{2}$ to form water.

Compared to other fuel cells, the main attractive features of solid oxide fuel cell systems are high efficiency, flexible choices of fuels and low emissions ${ }^{7,8}$. 
However, the historic cell performance and stability have limited SOFCs commercial applications. Researchers worked on studying electrode mechanisms to understand how and why electrode performance changes with cell operation conditions. New electrode materials were explored based on the mechanism understandings. Much of the investigations focused on the cathode because oxygen reduction is generally considered to be more difficult to activate than hydrogen oxidation.

\subsection{SOFCs Cathode}

As mentioned above, oxygen reduction occurs at the SOFC cathode and the overall reaction can be written by Kröger-Vink notation as:

$$
\frac{1}{2} O_{2}(g)+2 e^{\prime}+V_{o} \rightarrow O_{O}^{x}
$$

Materials which are used as SOFC cathodes must satisfy the following requirements ${ }^{9}$ :

- Adequate electronic and ionic conductivity.

- High catalytic activity for oxygen reduction.

- Chemical stability and relatively low interactions with the electrolyte.

- High compatibility with other cell components.

\subsubsection{SOFC cathode materials}

During the early stage of SOFC development, platinum and some other noble metals were used as the cathode materials. However, platinum is expensive and its compatibility with the electrolyte is not so good. Recently, less expensive perovskites which also possess the required properties have attracted much interest. 


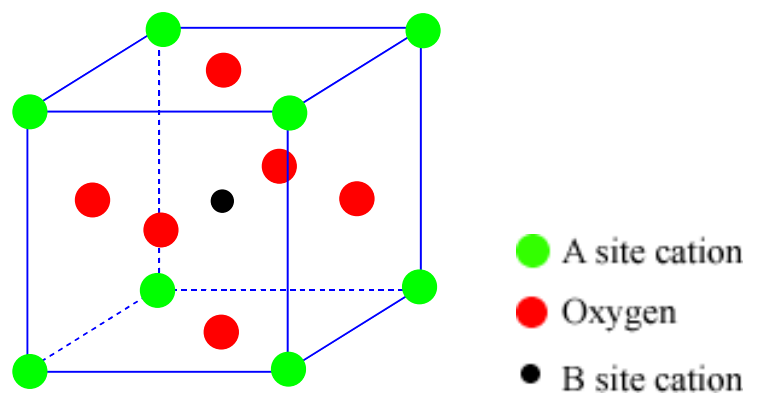

Figure 2.2 Schematic representation of lattice structure of perovskite, $\mathrm{ABO}_{3}$

Figure 2.2 reveals the lattice structure of $\mathrm{ABO}_{3}$ perovskite. In general, in the perovskite-type structure, B site element is closely bonded to six oxygen atoms with a strong covalent nature, while A site element coordinates to 12 oxygen ions with a strong ionic nature. Most of the perovskites can be considered as cathode materials except perovskites which have a low electronic conductivity such as $(\mathrm{La}, \mathrm{Sr})(\mathrm{Mn}, \mathrm{Fe}) \mathrm{O}_{3}, \mathrm{YCoO}_{3}$ or $(\mathrm{Y}, \mathrm{Ca}) \mathrm{FeO}_{3}{ }^{10}$.

$\mathrm{La}_{1-\mathrm{x}} \mathrm{Sr}_{\mathrm{x}} \mathrm{MnO}_{3 \pm \delta}$ (LSM) is one of the most widely used and researched cathode perovskite materials in SOFC. Sr-doped lanthanum perovskites show both large oxygen-excess under oxidizing atmosphere and large oxygen deficient when in reducing gas atmospheres ${ }^{11}$. In the oxygen deficient region oxygen vacancies are the main defects while in oxygen excess metal vacancies are the main defects. Although demonstrating good electrode properties for the YSZ electrolyte $^{12}$, the oxygen ion conductivity of LSM materials and its oxygen trace diffusion coefficient are undesirably low ${ }^{13}$. This poses practical limitations and restrictions to the application of LSM cathodes to SOFC operating at low temperature $\left(<800^{\circ} \mathrm{C}\right)$. 
The iron and cobalt-containing perovskite $\mathrm{La}_{1-\mathrm{x}} \mathrm{Sr}_{\mathrm{x}} \mathrm{Co}_{1-\mathrm{y}} \mathrm{Fe}_{\mathrm{y}} \mathrm{O}_{3 \pm \delta}$ (LSCF) is another candidate material for SOFC cathodes. Compared with LSM based materials, LSCF has higher ionic and electronic conductivity. Hence, sometimes LSM will be treated as an electronic conductor (EC) while LSCF is always a mixed ionic and electronic conductor (MIEC) ${ }^{14}$. Although the use of LSCF as SOFC cathode can effectively enhance the cell's performance, LSCF cathodes must be selected carefully because they have a higher thermal expansion coefficient (TEC) than the YSZ electrolyte ${ }^{15}$

To lower the operating temperature of SOFCs, Strontium-doped samarium cobaltite with composition $\mathrm{Sm}_{0.5} \mathrm{Sr}_{0.5} \mathrm{CoO}_{3}$ (SSC) has been studied recently. Its conductivity is much higher than LSCF and LSM at low temperature $\left(500-800^{\circ} \mathrm{C}\right){ }^{16,17}$. On the other hand, just as for LSCF, the TEC of SSC is too large for wholly adequate compatibility with the YSZ electrolyte. However, SSC is particularly compatible with GDC and LSGM ${ }^{18}$.

As illustrated above, the TEC of LSM is close to YSZ but its ionic conductivity is very low. The electronic and ionic conductivities of LSCF and SSC are high but their TEC are much higher than YSZ. Therefore, many alternative materials have been studied with the aim of increasing ionic conductivity and obtaining a TEC close to that of YSZ. For example, Fe-substituted lanthanum strontium cuprite $\left(\mathrm{La}_{0.7} \mathrm{Sr}_{0.3} \mathrm{Cu}_{0.4} \mathrm{Fe}_{0.6} \mathrm{O}_{3-\delta}\right)$ was reported to present a high conductivity and good thermal expansion match to $\mathrm{SDC}^{19} . \mathrm{Ba}_{0.5} \mathrm{Sr}_{0.5} \mathrm{Co}_{0.8} \mathrm{Fe}_{0.2} \mathrm{O}_{3-\delta}$ (BSCF) is another attractive cathode material for intermediate temperature $\mathrm{SOFCs}^{20}$. 


\subsubsection{SOFC cathode reaction mechanisms}

The cathode reaction mechanism is a fundamental issue for electrochemistry study. A thorough elucidation includes useful information on how to improve cell performance. Limited by the development of solid state ionic conductivity theories, researchers have matured SOFC cathode models over the past 20 years.

Aqueous solutions are the traditional domain of electrochemical system studies. Therefore, generally accepted conclusions and common investigation methods of aqueous system are the basis for SOFC cathode studies. However, the solid state system is more complex compared with the aqueous system. First, three phase boundary (3PB), where gas molecular, cathode and electrolyte materials meet, exists stably in SOFCs. At the same time, at the cathode side gas/cathode and cathode/electrolyte two-phase boundaries (2PB) also exist. The parallel 2PB and 3PB pathways provide competing routes for oxygen reduction. Second, in aqueous systems only ion diffusion occurs while for SOFC cathodes oxygen gas and ions will diffuse against the electrons. Finally, the double layer formed on the electrode is different. For aqueous system, usually the inactive ions in the solution will move to the electrode surface under the electrical field. However, on the surface of the SOFC cathode, adsorbed atomic oxygen or ions will form the double layer. 


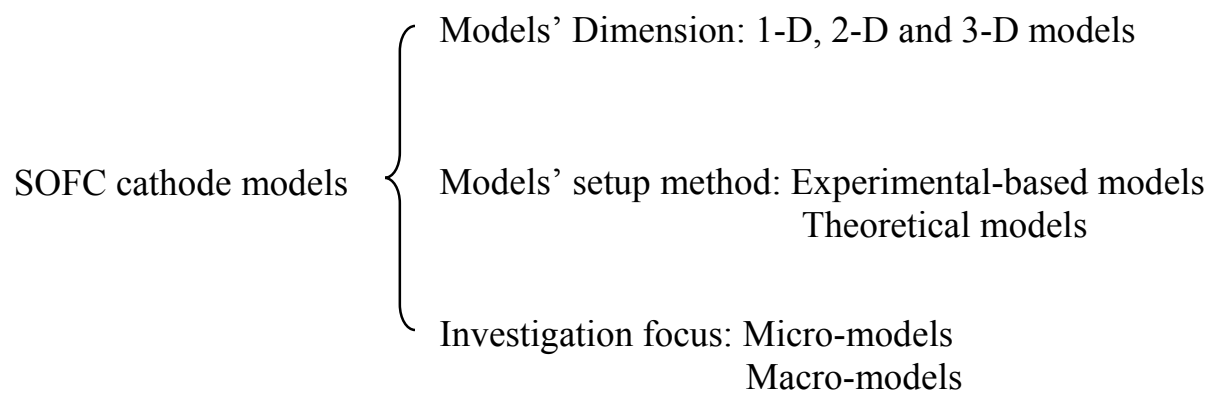

Figure 2.3 Different classifications of SOFCs cathode models

SOFC cathode models can be classified by different levels. As shown in Figure 2.3, according to the dimension of the model, there are 1-D, 2-D and 3-D models. Further, according to the model's setup method, some literature modeled SOFC cathodes in the viewpoint of theory while others utilized experimental data to set up their models. For example, some studies about three phase boundary had been done based on the SEM morphology results. Finally, according to different investigations, SOFC cathode models can be divided to micro-models and macromodels. The former studies the detailed cathode reaction steps and the latter one mainly considered the entire effect of the factors such as porosity, gas flow rate, temperature etc. Since our proposed work will focus on investigating the micro-scale oxygen transportation kinetics, development of SOFC cathode micro-models will be reviewed. Thus far, the most debated issue for micro-models is whether the reduction reaction is controlled by a chemical process or an electrochemical process.

\subsubsection{Pure chemical process}

Adler is one of the early researchers who treated the SOFCs cathode reaction as a pure chemical process. In the model developed in 1996, as shown in Figure 2.4, Adler et al. ${ }^{21}$ specified that the 
overall cathode reaction occurred via three separated interfacial reactions: (1) charge transfer of oxygen ion vacancies across the cathode material/electrolyte interface; (2) charge transfer of electrons across the current collector/cathode material interface; (3) chemical exchange of oxygen at the gas/cathode material interface. Due to the last specification, this model can only be valid for the mixed conductors with high rates of oxygen surface exchange, such as LSC.

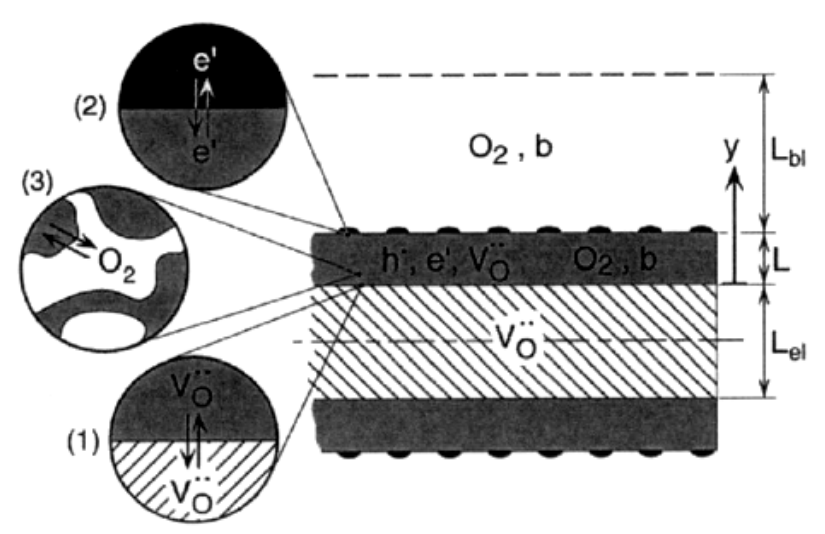

Figure 2.4 Cell geometry in Adler's model

The contributions to cathode kinetics were claimed as only the diffusion of oxygen and exchange of $\mathrm{O}_{2}$ at the mixed conductor/gas interface. To illustrate the chemical contribution, either one of the non-charge transfer steps would be considered as the rate limited step of the entire cathode reaction. Their models showed, when the surface exchange and solid state diffusion dominated, the total cell impedance would reduce to:

$$
Z=R_{\text {chem }} \sqrt{\frac{1}{1+j \omega t_{\text {chem }}}}
$$

When the gas phase diffusion was the limit, the total cell impedance was:

$$
Z=\frac{R_{\text {gas }}}{1-j \omega R_{\text {gas }} C_{\text {gas }}}
$$


$R_{\text {chem }}$ and $R_{\text {gas }}$ are characteristic resistances, $t_{\text {chem }}$ is a relaxation time of oxygen surface exchange and $C_{g a s}$ is the effective capacitance with gas phase diffusion polarization. Their results showed that the bulk properties of the cathode material would quantitatively affect the electrode kinetics. In order to verify this simulated result, Adler et al. compared the measured ac response of a symmetrical cell, which was composed of two $\mathrm{La}_{0.6} \mathrm{Ca}_{0.4} \mathrm{Fe}_{0.8} \mathrm{Co}_{0.2} \mathrm{O}_{3-\delta}$ electrodes and $\mathrm{Ce}_{0.9} \mathrm{Sm}_{0.1} \mathrm{O}_{2-\mathrm{x}}$ electrolyte, with the predicted results. As Figure 2.5 presents, the measured plots agreed well with the calculated results.

Additionally, in this model work, Adler brought out the concept of a characteristic distance which indicated the extension of the reaction zone beyond the three-phase boundary. The chemical resistances corresponding to different characteristic distances were calculated based on the model. The greatest extension distance was expected to be few microns.

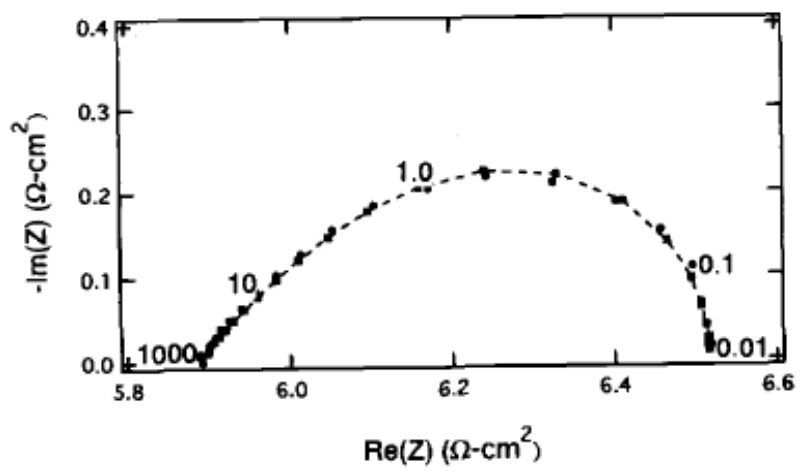

Figure 2.5 Plot of measured (circles) and calculated(squares) complex impedance of a symmetrical cell in air at $700^{\circ} \mathrm{C}$ 
As in the early mixed conductor cathode modeling, Adler's work offered valuable insight to the reaction mechanism. However, there also existed many arguable parts ${ }^{22}$. People doubted whether it's reasonable to define all the reduction reactions occurring at the electrode/gas interface. Besides, if the oxygen would be reduced, why is the oxygen surface exchange defined as a noncharge transfer reaction. Adler addressed these criticisms in a subsequent paper $^{23}$, where he argued that the oxygen reduction should only occur at the electrode/gas interface since matter can not pass through a truly three phase boundary. Furthermore, the processes of "chargetransfer" and "non-charge-transfer" had been defined. "Charge-transfer" represented any step that involves charged species and driven directly by gradients in electrical state and always occurred at a rate proportional to the current. On the other hand, "non-charge-transfer" processes involve neutral species or neutral combinations of species. Such processes are driven by gradients in chemical potential and occur at a rate independent of current.

Symmetrical cells under three different conditions were discussed in this paper ${ }^{23}$. Finally, the author concluded that the oxygen reduction was limited by the oxygen diffusion. And the diffusion process was independent with the electrochemical factors since molecular oxygen is neutral. The model ignored the effects of electron and oxygen vacancy concentrations inside the mixed conductor. As the main participants, they would certainly affect the oxygen reduction reaction. Furthermore, since those particles are chargeable, electrochemical conditions may control their diffusion process. Therefore, Adler's model may be more suitable for the period when the fuel cell starts running. 


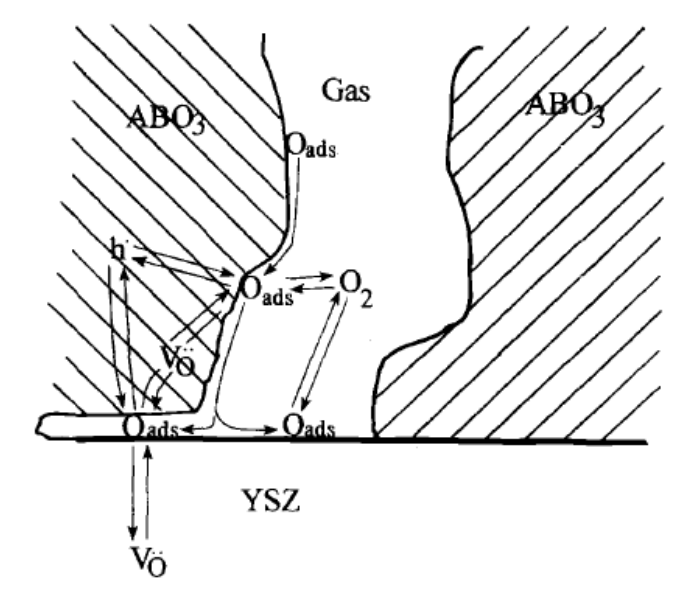

Figure 2.6 Sketch of Svensson's two step reactions cathode model

Svensson et al. developed a physical model to show the possible oxygen transport pathways on SOFC cathodes ${ }^{24,25}$. As shown in Figure 2.6, the first step of oxygen reduction is adsorption and desorption of neutral, monatomic oxygen at the gas/cathode and gas/electrolyte interfaces. Then the absorbed oxygen atom will combine with the vacancy to form lattice oxygen. The two step reactions can be written as:

$$
\begin{array}{r}
O_{2}(g)+2 a d s \stackrel{k_{a d s}}{\underset{k_{d e s}}{\longrightarrow}} 2 O_{a d s} \\
O_{a d s}+V_{o} \underset{k_{o}}{\stackrel{k_{r}}{\rightleftarrows}} a d s+2 h+O_{o}^{x}
\end{array}
$$

Svensson postulated that the interface between cathode and electrolyte contained intermediate oxygen species. Those species would either combine with the vacancies of the cathode material or combine with the vacancies of the electrolyte material. The reaction occurring at the gas/cathode interface was considered chemical in nature since no interfacial charge-transfer was involved, while the one occurring at the electrolyte surface was considered as an electrochemical 
process. Therefore, different from Adler's model, Svensson et al. introduced over-potential into their simulation to express the departure from equilibrium of the surface exchange reaction occurring at the electrolyte surface. According to Svensson's numerical results, a limiting current was predicted at high over-potential due to depletion of oxygen at the cathode/electrolyte interface, and they found there was a correlation between the limiting current and $P_{O_{2}}\left(i \propto P_{O 2}^{n}\right)$. When the exchange process was the limited rate step for the cathode reaction, the value of $n$ was between 0.58 and 0.74 . Smaller $n$ values $(0.26<\mathrm{n}<0.56)$ were predicted for a slow adsorption process. However, the two oxygen pathways in Svesson's model were considered in separate simulations.

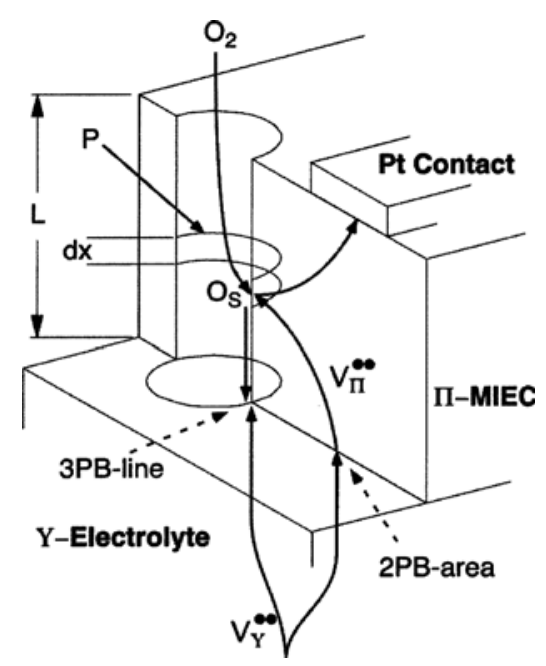

Figure 2.7 Cross-sectional schematic of the physical structure and chemical reactions occurring at porous SOFCs cathode

By the approach of Svesson, the influence of surface and bulk transport pathways for SOFC cathode can be described, but it is not possible to quantitatively compare the contribution to the total current by each path. To solve this problem, Coffey et al ${ }^{26}$ presented a continuum model which simultaneously considered both pathways, as illustrated in Figure 2.7. They considered that oxygen may transport through the triple-phase boundary (3PB) between the electrolyte, gas 
and cathode or the two-phase boundary (2PB) between the cathode and electrolyte. B-V equations for the surface over-potentials were taken as the boundary conditions in this model's simulation. Since the total voltage drop across the cathode-electrolyte interface is independent of the transport path chosen, the over-potential for the other path can be calculated by setting one path over-potential. However, although Coffey treated the reactions occurring at $3 \mathrm{~PB}$ and $2 \mathrm{~PB}$ interfaces as electrochemically motivated reactions, oxygen reduction occurring at the gas/cathode interface was considered as chemical reaction due to the fact that no net charge was gained or lost by the cathode.

In 2006, researchers from NASA set up a SOFC model for system controls and stability design ${ }^{27}$. The cathode reaction process was considered associated with oxygen absorption, desorption, diffusion and electronation. The charge transfer pathways can be presented as below,

$$
\begin{gathered}
O_{2}(g)+2 s \stackrel{k_{a d}, k_{d e s}}{\longrightarrow} 2 O_{a d} \\
O_{a d}+V_{O}^{\ddot{H}}+2 e^{-\stackrel{k_{l c}, k_{-l c}}{\longleftrightarrow}} O_{O}^{x}+s
\end{gathered}
$$

where $s$ is the concentration of vacant surface sites.

As we can see from the cathode mechanisms stated above, researchers considered the oxygen reduction as a pure chemical reaction because they thought there was no net charge transfer or charged intermediate at the interface. However, during the real oxygen reduction process charged intermediates are possibly formed. Furthermore, although there may be no net charge transfer at 
the reaction interface many researchers hold that the reaction should be considered as an electrochemical process since its reactants and products are charged particles.

\subsubsection{Electrochemical process}

Considering oxygen reduction is a reaction involving charged particles, many researchers hold that the cathode reaction of SOFC is an electrochemical process. It is well-accepted that the surface over-potential influences the entire cathode reaction process. Besides, the researchers also argue that oxygen reduction is not completed in one step which means some intermediates exist during the reaction process. Absorbed, charged intermediates will be affected by the surface over-potential.

Liu and Winnick investigated the reactions occurring on MIEC/gas interface. They considered oxygen reduction involving several intermediate ${ }^{28}$. Possible reaction pathways are shown below:

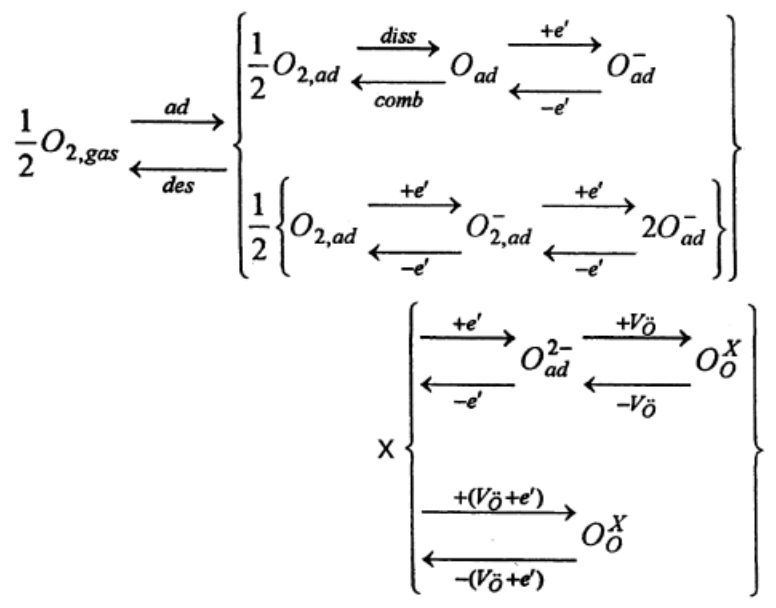

Figure 2.8 Possible oxygen reduction processes by Liu et al. 
Further, Liu et al. discussed the electrical state effects on the rate of interfacial reactions. Based on their analysis, the oxygen reduction rate depends critically on the electrical state of the MIEC/gas surface. Hence the reaction was considered as electrochemical.

F. H. van Heuveln ${ }^{29}$ considered the existence of $\mathrm{O}^{-}, \mathrm{O}_{2}^{-}$during the oxygen reduction process, and assumed the existence of three possible charge transfer pathways. $\mathrm{La}_{0.85} \mathrm{Sr}_{0.15} \mathrm{MnO}_{3}$ cathode was prepared by tape casting on a pre-sintered YSZ pellet. Electrochemical resistance and Tafel plots were measured to verify van Heuveln's model. The experimental results indicated that diffusion of $\mathrm{O}_{\mathrm{ad}}{ }^{-}$species along the LSM surface to 3PB area will compete with charge-transfer at low overpotential. The diffusion limitation disappears at high cathodic over-potentials. On the other side, the model's simulation results also show that the diffusion process is influenced mainly by the current. However, in van Heuveln's model, the reduction occurring at the gas/cathode interface was ignored. The MIEC was treated similar to the metal electrode.

Model 1

$$
\begin{aligned}
& O_{2}(g) \rightarrow 2 O_{a d} \\
& O_{a d}+e \rightarrow O_{a d}^{-} \\
& O_{a d}^{-} \rightarrow O_{T P B}^{-} \\
& O_{T P B}^{-}+e+V_{o}^{*} \rightarrow O_{o}^{x} \\
& O_{2}(g)+e \rightarrow O_{2, a d}^{-} \\
& O_{2, a d}^{-}+e \rightarrow 2 O_{a d}^{-} \\
& O_{a d}^{-} \rightarrow O_{T P B}^{-} \\
& O_{T P B}^{-}+e+V_{o}^{*} \rightarrow O_{o}^{x}
\end{aligned}
$$




$$
\begin{array}{ll} 
& O_{2}(g)+e \rightarrow O_{2, a d}^{-} \\
& O_{2, a d}^{-} \rightarrow O_{2, T P B}^{-} \\
& O_{2, T P B}^{-}+e+V_{o} \rightarrow O_{O}^{x}+O_{a d} \\
& O_{a d}+e \rightarrow O_{a d}^{-} \\
& O_{a d}^{-}+e+V_{o}^{*} \rightarrow O_{o}^{x}
\end{array}
$$

Mitterdorfer ${ }^{30}$ also thought $\mathrm{O}^{-}, \mathrm{O}_{2}^{-}$may exist during the reduction process. He developed a physical model to explain oxygen transport from the LSM cathode to the YSZ electrolyte. The reactions can be described as follow:

$$
\begin{aligned}
& \mathrm{O}_{2}(g)+e^{-}+s \Leftrightarrow O_{2}^{-}(a d) \\
& O_{2}^{-}(a d)+e^{-} \Leftrightarrow 2 O^{-}(a d)
\end{aligned}
$$

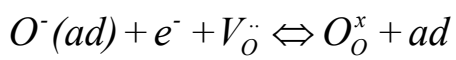

In Mitterdorfer's model, LSM had been considered as pure electronic conductivity. The oxygen ions were assumed as formed only at the 3PB area.

Chan et al. developed a micro-model for an LSM electrode ${ }^{31}$. All possible polarizations which govern the complex interdependency among the transport phenomena, electrochemical reaction and microstructure of the electrode and their combined effect on the cathode over-potential under different operating conditions had been considered in this model. They claimed that when the applied oxygen partial pressure was lower than $0.1 \mathrm{~atm}$, a third arc can be seen in the low frequency band of impedance spectra which is due to gas phase diffusion. 
Chan applied the reaction steps of van Heuveln's model 1 to develop their model. The difference is that Chan et al. established a correlation between the microstructure and the performance of the cathode. According to the simulation results, it was found that larger particle size requires a thicker cathode for reduced cathode over-potential. Furthermore, the current density and oxygen partial pressure were found to not affect the optimal electrode thickness value. However, current density and oxygen partial pressure will affect the optimal particle size.

Bilge Yildiz presented a two dimensional physical model that includes the effect of both surface and bulk pathways under different operating conditions and electrode configurations ${ }^{32}$. The possible surface and bulk pathways were shown below, and AC impedance spectra of LSM electrode were applied to verify Yildiz's model:

$$
\begin{array}{ll} 
& O_{2(g)} \Leftrightarrow 2 O_{a d, s} \\
& O_{a d, s}+e^{-} \Leftrightarrow O_{s}^{-} \\
& O_{s}^{-} \Leftrightarrow O_{3 P B}^{-} \\
& O_{3 P B}^{-}+e^{-} \Leftrightarrow O_{3 P B}^{2-} \\
& O_{3 P B}^{2-}+V_{\ddot{o}}^{Y} \Leftrightarrow O_{O}^{Y} \\
& \\
& O_{2(g)} \Leftrightarrow 2 O_{a d, s} \\
& O_{a d, s} \Leftrightarrow O_{3 P B} \\
& O_{3 P B}+e^{-} \Leftrightarrow O_{3 P B}^{-} \\
& O_{3 P B}^{-}+e^{-} \Leftrightarrow O_{3 P B}^{2-} \\
& O_{3 P B}^{2-}+V_{\ddot{o}}^{Y} \Leftrightarrow O_{O}^{Y}
\end{array}
$$

To prove that the intermediates are likely to exist during the oxygen reduction process, Liu et al. investigated the oxygen reduction process on a silver electrode surface using the first-principles 
calculations based on the density functional theory and pseudo-potential method ${ }^{33}$. The calculation results suggest that the oxygen reaction on silver cathode can be described like this:

$$
O_{2}(g)(+e) \rightarrow O_{2}^{-}(+e) \rightarrow O_{2}^{2-}(+2 e) \rightarrow 2 O^{2-}
$$

Since in this work a metal electrode has been studied, the calculation results show that the oxygen reduction and the incorporation of the dissociated $\mathrm{O}$ ions in the oxide electrolyte prefer the 3PB region. For MIEC electrodes, due to the fact that oxygen vacancy may exist inside the material, the reduction process will not only occur near 3PB region. However, although this work offers some evidence for the intermediates, it's not so convincing that they will appear in perovskite MIEC. Therefore researchers still examined new ways to verify the existence of the intermediates and to confirm the intermediates appearing during the reduction process.

Since researchers considered the cathode reaction as an electrochemical process, surface overpotential needs to be introduced into their simulations. To make a clear idea on this issue, first we need to know what the over-potential is and how it is generated. Over-potential refers to the potential difference between a half-reaction's thermodynamically determined reduction potential and the potential at which the redox event is experimentally observed. For an aqueous system, the over-potential is formed at the electrode/electrolyte interface. For the SOFC cathode, the over-potential exists not only at the electrode/electrolyte interface but also at the gas/electrode interface and the TPB area. And there are three different parts for the over-potential: overpotential caused by the material's resistance, over-potential caused by the surface exchange process and over-potential caused by the oxygen ion diffusion. 

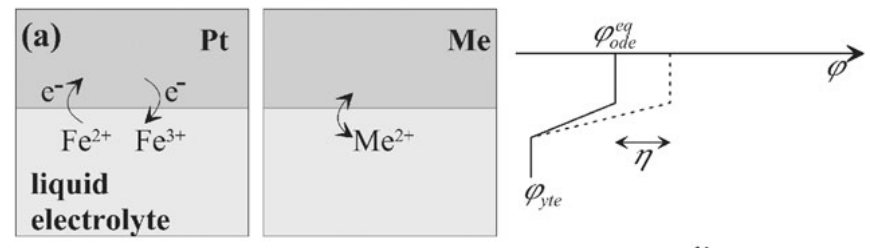

(b)
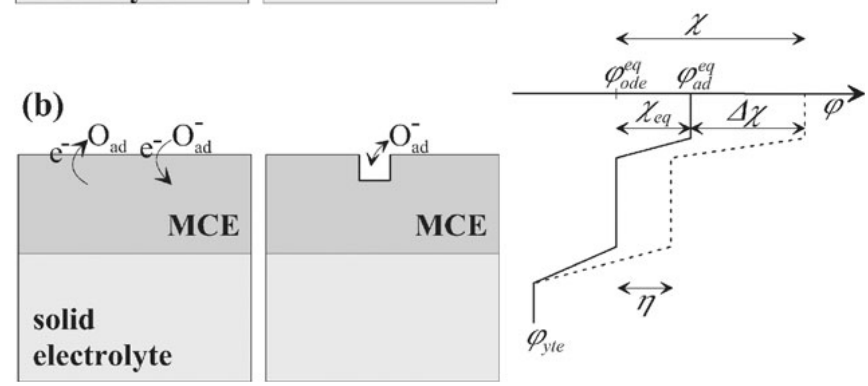

Figure 2.9 Sketches of electron and ion transfer reactions at (a) metal/liquid electrolyte and (b) mixed conducting electrode (MCE)/solid electrolyte interfaces

J. Fleig discussed MIEC surface over-potential which is caused by surface charge transfer ${ }^{34}$. Figure 2.9 depicts the over-potential difference between a liquid electrolyte system and the SOFC cathode.

For a metal electrode in an aqueous electrochemical system, electron and ion transfer only occur at the electrode/electrolyte interface so the over-potential can only be formed at the electrode/electrolyte interface. However for mixed conducting electrode, electron and ion transfer also occur at the electrode/gas interface. Hence it's necessary to introduce the term of surface over-potential change $\Delta \chi$ into the simulation. Fleig discussed the application of $\Delta \chi$ under electron transfer step control and ion transfer step control conditions. He also studied the relationship of MCE surface over-potential $(\Delta \chi)$ and electrode/electrolyte interface over-potential $(\eta)$ 


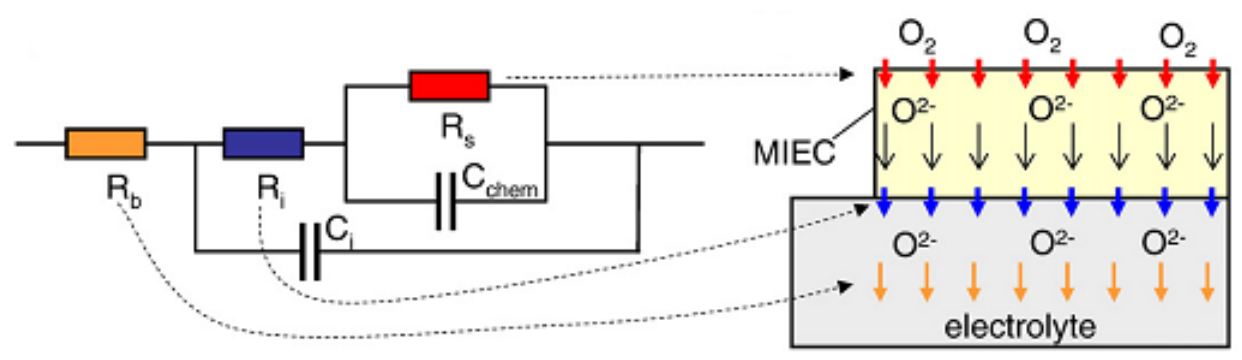

Figure 2.10 An equivalent circuit of the MIEC electrode and the sketch of the oxygen reduction process

In later work, the equivalent circuit based on the understanding of the oxygen reduction pathways was presented, as Figure 2.10 shows ${ }^{35}, \quad 36, \mathrm{La}_{0.6} \mathrm{Sr}_{0.4} \mathrm{Co}_{0.8} \mathrm{Fe}_{0.2} \mathrm{O}_{3-\delta}$, $\mathrm{Ba}_{0.5} \mathrm{Sr}_{0.5} \mathrm{Co}_{0.8} \mathrm{Fe}_{0.2} \mathrm{O}_{3-\delta}$ and $\mathrm{Sm}_{0.5} \mathrm{Sr}_{0.5} \mathrm{CoO}_{3-\delta}$ thin films were applied to study the oxygen reduction. Thin films and patterned electrodes are usually used for investigating over-potential effects because it is possible to precisely control the geometry related to the triple phase boundary and bulk reaction pathways. Based on the ac and dc resistance measurements, Fleig claimed that the capacitance in Fig. 10 was a constant phase element $\left(Q^{-1}(i \omega)^{-n}\right)$ with exponents close to one. And they found for the investigated materials, the oxygen exchange reaction on the MIEC surface limited the kinetics of the overall oxygen reduction reaction. On the other hand, according to the experimental results, $\mathrm{Ba}_{0.5} \mathrm{Sr}_{0.5} \mathrm{Co}_{0.8} \mathrm{Fe}_{0.2} \mathrm{O}_{3-\delta}$ exhibited the lowest surface-related polarization resistance compared to the other two materials. 


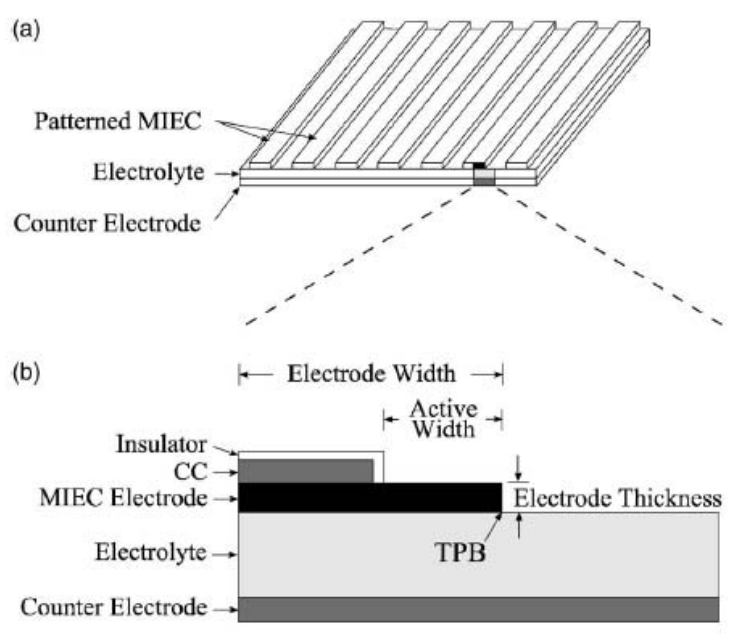

Figure 2.11 Sketch of 2-D model

(a) patterned electrode array (b) symmetric 2-D cross-sectional model domain

Liu developed a 2-D model based on Fleig's theory of MIEC surface over-potential ${ }^{37}$. Figure 2.11 depicts their model geometry. As Figure $2.11 \mathrm{~b}$ shows, the dashed-line box is the two dimensional investigated region.

The gas exposure surface reactions were assumed as below:

$$
\begin{gathered}
\frac{1}{2} \mathrm{O}_{2}+\mathrm{s} \rightarrow \mathrm{O}_{\mathrm{ads}}^{\prime}+\mathrm{h} \\
\mathrm{O}_{\mathrm{ads}}^{\prime}+\mathrm{V}_{\mathrm{O}} \rightarrow \mathrm{O}_{\mathrm{o}}^{\mathrm{x}}+\mathrm{h}+\mathrm{s}
\end{gathered}
$$

The reaction rate had been discussed in their previous work ${ }^{38}$. The simulation results show that under low over-potential the ionic transportation will be the rate limiting step which means low over-potential $(50 \mathrm{mV})$ doesn't affect oxygen reduction. Under high over-potential $(750 \mathrm{mV})$, the 
sheet resistance will control the reaction rate which means that the surface exchange step will be the rate limiting step.

\subsubsection{Summary}

The literature reviewed in this part has shown that the oxygen reduction mechanism in the cathode of an SOFC is complex. Although different experimental methods have been utilized to verify model results, differences of opinion still exist among researchers. Several main debatable issues about SOFC cathode reaction mechanisms are summarized as below:

(1) Reaction intermediates: Different sub-reactions were offered by researchers for the same overall oxygen reduction reaction. Adsorbed oxygen atoms, $\mathrm{O}^{-}$and $\mathrm{O}_{2}^{-}$all possibly appear via one or more steps. The surface over-potential needs to be considered when there are chargeable intermediates during the reduction process. However, based on the presently investigated literature, it is still difficult to determine which intermediates actually exist in the reaction.

(2) Reaction region: Generally, the 3PB region where cathode, electrolyte and gas connect together was supposed to be the main reaction region. However, due to the fact that the cathode material is a mixed ionic and electronic conductor, oxygen reduction also may occur at gas/cathode interface. Therefore, two charge transfer pathways will co-exist and may compete under different operation conditions.

(3) Rate limited steps: The global oxygen reduction can be divided into several sub-reactions according to the understandings of oxygen reduction mechanism. Besides the reduction reactions, oxygen diffusion is the other step for the cathode reaction process. Rate limited step determination under different conditions is important for optimizing the cathode and improving the cell efficiency. 
(4) Geometry factors: Cathode geometrical factors such as porosity, tortuosity and TPB length are important for SOFC cathode modeling. They will affect the gas diffusion process and the cell performance. Due to the fact that the shape and the distribution of the pores inside SOFCs cathode are random, it's difficult to get an accurate simulation result compared to the real operating conditions. Some researchers applied a factor parameter to minimize the errors on ignoring the geometry effects. Most researchers used a computer to build up a random microstructure with some input parameters. An effective way is to get part of the microstructure information from the experimental method first, then use a computer program to simulate the entire cathode reaction process.

\subsubsection{SOFC cathode infiltration}

Based on the understanding of physics and chemistry governing SOFC cathode reactions, there are two principal approaches to improve the performance. One strategy is to develop new cathode material with both high electronic and ionic conductivity. By increasing oxygen bulk ionic transport, the active region will be extended to the electrode surface and the oxygen reduction kinetics will be improved. However, considering the requirements for electrode compatibility and stability with other components, it is not a simple issue for developing new materials of SOFCs cathode. Another possible way is to combine the cathode electrode materials with a more ionically conducting material. Wet impregnation, which is carried out by infiltrating porous cathode with nitrates by capillary action and then decomposing the nitrate solutions by heat treatment, is a common way to implement composite SOFCs cathode. It has been reported that the impregnation of nanoscale particles such as $\mathrm{Pd}^{39}$, ceria ${ }^{40,41}, \mathrm{Ag}^{42,43}, \mathrm{Co}_{3} \mathrm{O}_{4}{ }^{44}$ and bismuth oxide $^{45}$ can greatly reduce the polarization resistance of the cathode. 
Besides the electro catalytic promoters, high ionic conductivity materials are also infiltrated to improve the cathode performance. Gorte ${ }^{46}$ et al. infiltrated LSM/LSC composite cathode on a porous YSZ matrix with aqueous nitrates solution. The LSM-YSZ composites were prepared with $30 \mathrm{wt} \% \mathrm{LSM}$ and were co-sintered to $1323 \mathrm{~K}$, after which $10 \mathrm{wt} \%$ of LSCo was added by wet impregnation using aqueous solutions of the nitrate salts. The maximum power density increased to above $400 \mathrm{~mW} / \mathrm{cm}^{2}$ and the non-ohmic losses decreased from greater than $0.6 \Omega \cdot \mathrm{cm}^{2}$ to approximately $0.3 \Omega \cdot \mathrm{cm}^{2}$. Xiong ${ }^{47}$ et al. infiltrated samarium doped $\mathrm{CeO}_{2}(\mathrm{SDC})$ nano particles into $\left(\mathrm{Pr}_{0.7} \mathrm{Ca}_{0.3}\right)_{0.9} \mathrm{MnO}_{3-\delta}(\mathrm{PCM})$ cathode. The cell with $2.6 \mathrm{mg} / \mathrm{cm}^{2} \mathrm{SDC}$ infiltrated in cathode showed the maximum power density of $580 \mathrm{~mW} / \mathrm{cm}^{2}$ compared with $310 \mathrm{~mW} / \mathrm{cm}^{2}$ of the cell without impregnation at $850^{\circ} \mathrm{C}$. Xia et al. ${ }^{41}$ fabricated $\left(\mathrm{La}_{0.85} \mathrm{Sr}_{0.15}\right)_{0.9} \mathrm{MnO}_{3-\delta}(\mathrm{LSM})-$ $\mathrm{Sm}_{0.2} \mathrm{Ce}_{0.8} \mathrm{O}_{1.9}(\mathrm{SDC})$ composite electrodes by wet impregnation. The impregnation resulted in a significant reduction of the interfacial resistance at $700^{\circ} \mathrm{C}$ from $5.43 \Omega \cdot \mathrm{cm}^{2}$ from pure LSM cathode to $0.233 \Omega \cdot \mathrm{cm}^{2}$ for an LSM-SDC50 composite cathode. Lee et al. ${ }^{48}$ studied dual-phased functional layer containing $\mathrm{Sm}_{2} \mathrm{O}_{3}$-doped $\mathrm{CeO}_{2}$ (SDC) and $\mathrm{La}_{0.6} \mathrm{Sr}_{0.4} \mathrm{Co}_{0.2} \mathrm{Fe}_{0.8} \mathrm{O}_{3}-\delta$ (LSCF) backbone infiltrated with either $\mathrm{La}_{0.6} \mathrm{Sr}_{0.4} \mathrm{CoO}_{3}$ (LSC) or $\mathrm{La}_{1.97} \mathrm{Sr}_{0.03} \mathrm{Zr}_{2} \mathrm{O}_{7}$ (LSZ) cathodes.

Although wet impregnation is a well-known technique in the preparation of heterogeneous catalysts, the wide application of wet impregnation technique in SOFCs is a recent phenomenon. And compared to the single phase cathode, the infiltrated composite cathode is a more complex system. Therefore, no confirmed reaction model for composite SOFCs cathode had been set up. It is well accepted that the infiltrated material can extend TPB (triple phase boundary) length and promoted oxygen reduction. However, rare work on investigating oxygen transport behavior, 
which is also a key step of the electrode reaction process, can be found from references. If we assume oxygen reduction for the composite cathode mainly occurred at the infiltrated material/atmosphere (2PB) and infiltrated material/backbone/atmosphere (3PB) range, there will be two steps for oxygen ion transportation. First, oxygen ion will be transferred from the infiltrated material to the cathode backbone. Then it will diffuse inside the backbone material and further transferred to electrolyte. At the same time, $\mathrm{O}^{-}$surface diffusion may also exist. Since it can offer useful information on impregnation process optimization, study on how the impregnation technique will affect the two oxygen transport pathways is very important and necessary. In this proposed research work, a global evaluating method for composite SOFC cathodes will be developed.

\subsection{Electrical conductivity Relaxation (ECR)}

\subsubsection{Theoretical background}

The conductivity relaxation technique involves measurement of time variant electrical conductivity of a sample after a stepwise change in the ambient oxygen partial pressure. To determine oxygen surface exchange $(k)$ and bulk diffusion coefficients $(D)$, a correlation between a mean conductivity and the corresponding mean non-stoichiometry had been established ${ }^{49}$.

The possible defects in $\mathrm{ABO}_{3}$ perovskite include electrons $\left(e^{\prime}\right)$, holes $\left(h^{*}\right)$, oxygen vacancies $\left(V_{O}^{\bullet}\right)$, cation vacancies $\left(V_{A}^{\prime \prime}\right.$ and $\left.V_{B}^{\mathrm{m} \prime \prime}\right)$ and acceptor background impurities $\left(I^{\prime}\right)$. Based on overall charge neutrality, we have:

$$
n+2\left[V_{A}^{\prime \prime}\right]+4\left[V_{B}^{\prime \prime \prime \prime}\right]+\left[I^{\prime}\right]=p+2\left[V_{O}^{\bullet}\right]
$$


where $n, p$ and $[j]$ are the concentration of electrons, holes and ionic defects of type $j$. By defining the effective concentration of acceptors as

$$
\left[A^{\prime}\right]=2\left[V_{A}^{\prime \prime}\right]+4\left[V_{B}^{\prime \prime \prime \prime}\right]+\left[I^{\prime}\right]
$$

The oxygen non-stoichiometry, $\delta$, is then given as:

$$
\frac{N_{A} \delta}{V_{m}}=\left[V_{O}^{\ddot{\prime}}\right]-\frac{1}{2}\left[A^{\prime}\right]=\frac{1}{2}(n-p)
$$

where $N_{A}$ and $V_{m}$ represent the Avogadro's constant and the lattice molecular volume.

When the material conduction type is in the regime of exclusively $\mathrm{p}$ type $\left(\sigma_{p} \approx \sigma_{e l}\right)$, equation 2.21 takes the form:

$$
\frac{N_{A} \delta}{V_{m}}=-\frac{p}{2}=-\frac{\sigma_{p}}{2 e \mu_{p}}
$$

And when it's in the regime of exclusively $\mathrm{n}$ type $\left(\sigma_{n} \approx \sigma_{e l}\right)$,

$$
\frac{N_{A} \delta}{V_{m}}=\frac{n}{2}=\frac{\sigma_{n}}{2 e \mu_{n}}
$$

Assuming that the mobility, $\mu_{p}$ and $\mu_{n}$, are independent of the non-stoichiometry or oxygen partial pressure at a certain temperature, the following correlation can be established ${ }^{49}$ :

$$
\frac{\delta(t)-\delta(0)}{\delta(\infty)-\delta(0)}=\frac{\sigma(t)-\sigma(0)}{\sigma(\infty)-\sigma(0)}
$$

$\delta(0), \delta(\infty)$ and $\delta(\mathrm{t})$ separately stand for initial, final and time-related non-stoichiometry values. $\sigma(0), \sigma(\infty)$ and $\sigma(\mathrm{t})$ are conductivities corresponding to $\delta(0), \delta(\infty)$ and $\delta(\mathrm{t})$ respectively.

And since the non-stoichiometry can stand for oxygen concentration inside the material, similar relation between oxygen concentration and conductivity can be established: 


$$
\frac{\mathrm{C}(t)-\mathrm{C}(0)}{\mathrm{C}(\infty)-\mathrm{C}(0)}=\frac{\sigma(t)-\sigma(0)}{\sigma(\infty)-\sigma(0)}
$$

where $C(0), C(\infty)$ and $C(t)$ are conductivities corresponding to $\sigma(0), \sigma(\infty)$ and $\sigma(t)$, respectively. Solid phase equilibrium will be restored when the surface exchange is equal to the diffusion flux which is presented by equation 2.26 :

$$
-D \partial C /\left.\partial x\right|_{x= \pm a}=k[C(\infty)-C(t)]
$$

Based on this boundary condition and Fick's second law $\left(\frac{\partial C}{\partial t}=D \frac{\partial^{2} C}{\partial x^{2}}\right)$, a solution is determinable for a pellet sample ${ }^{50}$ :

$$
\frac{\sigma(t)-\sigma(0)}{\sigma(\infty)-\sigma(0)}=1-\sum_{n=1}^{\infty} \frac{2 L^{2} \exp \left(-b_{n}^{2} D t / a^{2}\right)}{b_{n}^{2}\left(b_{n}^{2}+L^{2}+L\right)}
$$

In equation 2.27:

$$
L=\frac{a}{l_{c}}=\frac{a k}{D}=b_{n} \tan b_{n}
$$

Here, $a[\mathrm{~cm}]$ is the half thickness of the tested pellet sample in the diffusion solution. And $L[-]$ is a unit-less parameter from which the characteristic thickness $l_{c}[\mathrm{~cm}]$ can be derived as shown in equation 2.28. $b_{n}$ is a parameter generated during the process solving Fick's second law. Its value can be determined by Newton's method using equation 2.28. The importance of the characteristic thickness has been described previously ${ }^{51}$ as a measure of the membrane thickness where the transition occurs between predominant control by bulk diffusion and surface exchange. If $0.1<L<10$, the oxygen transport process is considered to proceed under mixed control ${ }^{49}$. 


\subsubsection{IEDP method}

Two experimental methods were used to characterize oxygen transportation for SOFC cathodes at present. Aside from ECR, the other method is known as Isotope Exchange Depth Profile $(I E D P)^{52}$ method. Isotope ${ }^{18} \mathrm{O}$ is used in the experiment and the diffusion profile within the sample was determined by Secondary Ion Mass Spectrometry ${ }^{53}$. IEDP and ECR are both set up based on Fick's second law. For IEDP method, the sample usually will be first annealed in the labeled ${ }^{16} \mathrm{O}_{2}$ atmosphere for a time that is approximately one order of magnitude greater than the tracer anneal time, to ensure the sample is in chemical equilibrium in the desired temperature and atmosphere. Then the sample will be quenched to room temperature and reheated with the ${ }^{18} \mathrm{O}_{2}$ gas. Then ${ }^{18} \mathrm{O}$ penetration profile will be determined by SIMS. During this process, the rate of isotope exchange across gas/solid interface is assumed to be directly proportional to the difference in isotope concentration between the gas and the solid. This leads to the boundary condition:

$$
-\left.D \frac{\partial C}{\partial x}\right|_{x=0}=k\left(C_{s}-C_{g}\right)
$$

$\mathrm{C}_{\mathrm{g}}$ and $\mathrm{C}_{\mathrm{s}}$ refer to the ${ }^{18} \mathrm{O}$ fraction in the gas phase and at the sample surface respectively ${ }^{54,55,56 \text {, }}$ $57,58$.

The solution of Fick's second law can be obtained using this boundary condition:

$$
\frac{C(x, t)-C_{n a}}{C(x)-C_{n a}}=\operatorname{erfc}\left(\frac{x}{2 \sqrt{D^{*} t}}\right)-\exp \left(h x+h^{2} D^{*} t\right) \operatorname{erfc}\left(\frac{x}{2 \sqrt{D^{*} t}}+h \sqrt{D^{*} t}\right)
$$


Table 2.1 Two experimental methods for oxygen transport characterization

\begin{tabular}{l|l|l|l}
\hline Method & $\Delta P_{\mathrm{O}_{2}}$ & Obtained parameter & Advantages \\
\hline IEDP & No & Self diffusion coefficient (D*) & $\begin{array}{l}\text { More direct and accurate for oxygen } \\
\text { concentration detection }\end{array}$ \\
\hline ECR & Yes & Chemical diffusion coefficient (D) & $\begin{array}{l}\text { Economic; Testing condition is close to the real } \\
\text { fuel cell operation condition }\end{array}$ \\
\hline
\end{tabular}

The two experimental methods were compared in Table 2.1. It can be found out that although SIMS can detect oxygen concentration very accurately, ECR testing results are more meaningful for SOFC cathode investigation since the oxygen transport is driven by oxygen partial pressure gradient, which is close to SOFCs operation condition, rather than isotope concentration difference.

\subsubsection{Application and outlook}

Due to its advantages, the ECR technique had been widely used on oxygen transport characterization for SOFC cathode materials ${ }^{59,60,61,62}$. Yasuda ${ }^{63}$ et al. investigated $\mathrm{LaCrO}_{3}$ with electrical conductivity relaxation method in $\mathrm{CO} / \mathrm{CO}_{2}$ atmospheres. Oxygen diffusion coefficient was found increasing with decrease of oxygen partial pressure due to the corresponding change in the concentration of the moving species. Yasuda ${ }^{64}$ also utilized the $E C R$ technique to study $\mathrm{La}_{1-\mathrm{x}} \mathrm{Sr}_{\mathrm{x}} \mathrm{MnO}_{3-\delta}(\mathrm{x}=0.05,0.10,0.15$ and 0.20$)$. A defect model considering the formation of association pairs between divalent manganese ions and oxygen vacancies was proposed to elucidate the non-stoichiometric behavior of LSM. 
J. A. Kilner ${ }^{65}$ discussed the measurement of oxygen transport by using the relaxation technique. Several emphases regarding the testing process had been placed based on Kilner's work. First, the oxygen partial pressure change should occur as quickly as possible. Besides, the oxygen partial change step should be small since the diffusion and surface reaction coefficients do not behave linearly with $P_{\mathrm{O}_{2}}$.

Adler ${ }^{66}$ et al. investigated $\mathrm{CO}_{2}$ atmosphere effects on oxygen transport in $\mathrm{La}_{0.6} \mathrm{Sr}_{0.4} \mathrm{Fe}_{0.8} \mathrm{Co}_{0.2} \mathrm{O}_{3-\delta}$ material by $E C R$ technique. The results showed that at $750^{\circ} \mathrm{C}$, exposure to $\mathrm{CO}_{2}$ atmosphere decreased the surface exchange rate by a factor of 2 . It may be because carbonate formation on the oxide surface hindered the surface exchange reaction.

Besides utilization for oxygen transport behavior characterization, researchers also worked on improving the ECR technique itself. Otter derived equations which describe the transient response in conductivity relaxation experiments, taking into account the time correction for reactor flushing ${ }^{67}$. Wang et al. studied the effects of oxygen partial pressure step change together with the role of oxygen vacancy concentration on the relaxation kinetics ${ }^{68}$. However, data fitting method for ECR testing was ignored. Cox-Galhotra ${ }^{69}$ et al. addressed the low reliability of the fitting procedure that seeks to simultaneously determine oxygen surface exchange and bulk diffusion coefficients by analyzing single conductivity relaxation data set. The same phenomenon was detected at the beginning of our work. Galhotra studied sample grain size and surface roughness effects on the fitted kinetic parameters, but no concrete explanations were generated. Therefore, in this work, we will first improve the $E C R$ data analysis method. 
Moreover, a developed diffusion model for the ECR technique will be applied to characterize oxygen transport property in SOFC cathode materials of typical composition. 


\section{Experimental capabilities}

\subsection{Electrical conductivity relaxation testing system}

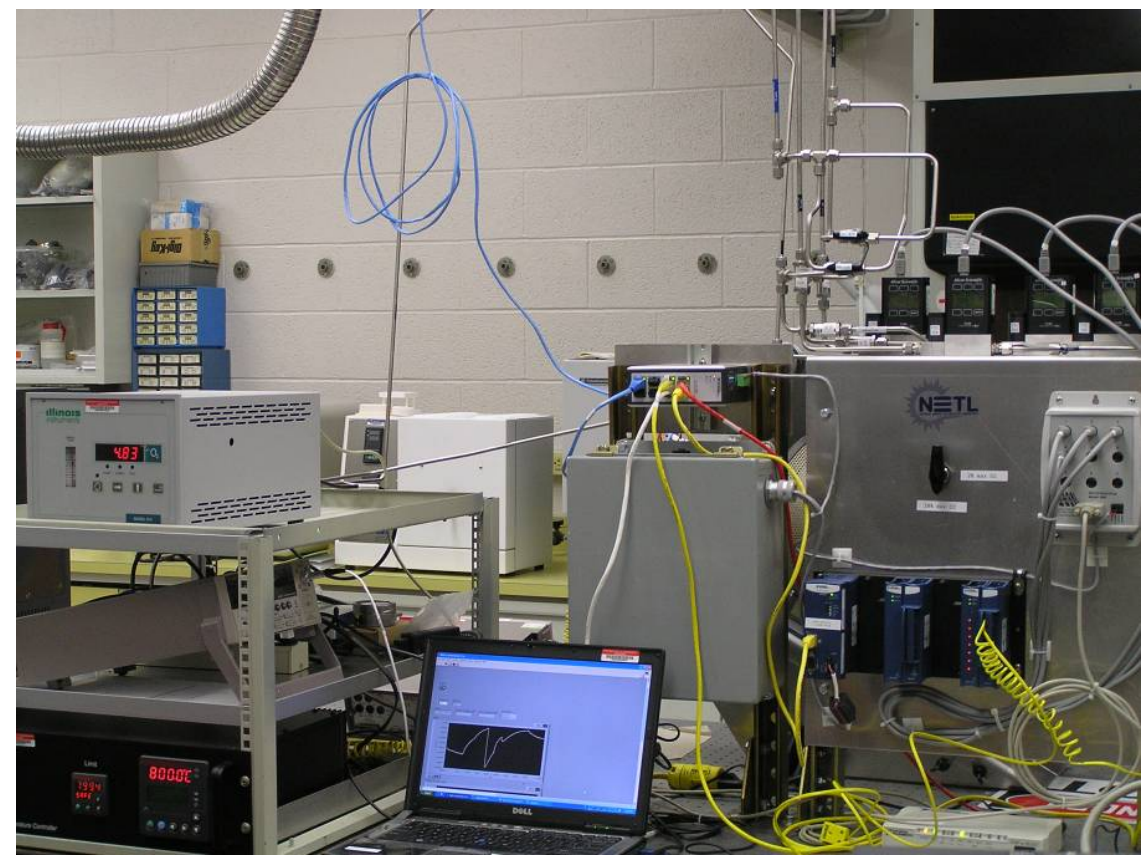

Figure 3.1 Electrical conductivity relaxation testing system

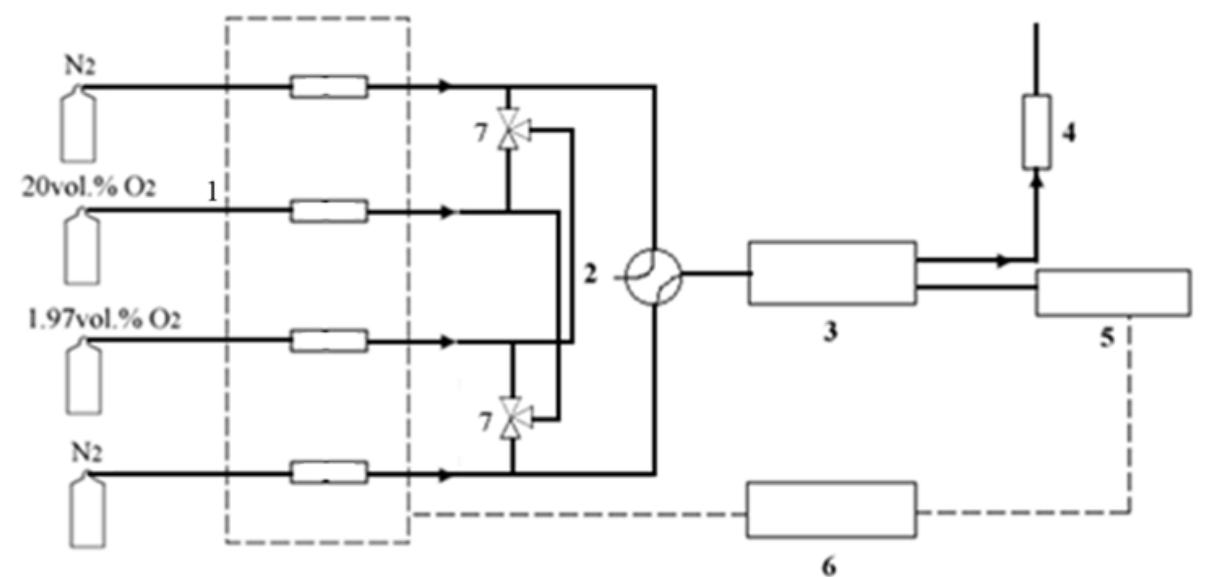

Figure 3.2 Schematic representation of the conductivity relaxation set-up

1. Mass flow controller 2. Four-way valve 3. Furnace 4. Oxygen analyzer 5. Nano-voltmeter and current source meter 6 . Computer 7 . Three way valve 
The electrical conductivity relaxation system was built up in our lab, as shown in Figure 3.1. A Keithley 2400 was used to apply constant current and the corresponding voltage was measured by a Keithley 2182A. The oxygen partial pressure was monitored with an electrochemical oxygen Analyzer (Model 810 Oxygen Analyzer, Illinois Instrument Inc.). Gas flow rate and other operating point measurements were handled by a National Instruments data acquisition controlled with LabVIEW software.

\subsection{Manufacture equipment for ECR samples and SOFC cathodes}

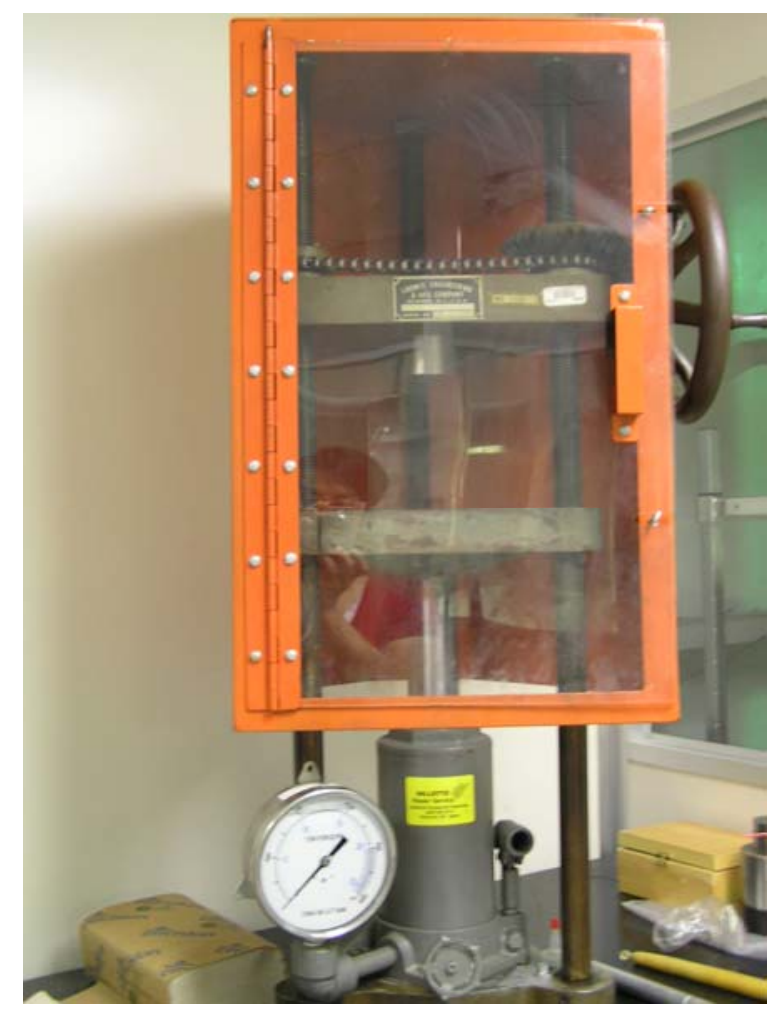

Figure 3.3 Manual Presser

LSCF powder is pressed to form a disk shape using a manual presser as shown in Figure 3.3. The pressed pellet is then sintered at $1350^{\circ} \mathrm{C}$ for $2 \mathrm{~h}$ in a high temperature furnace. 


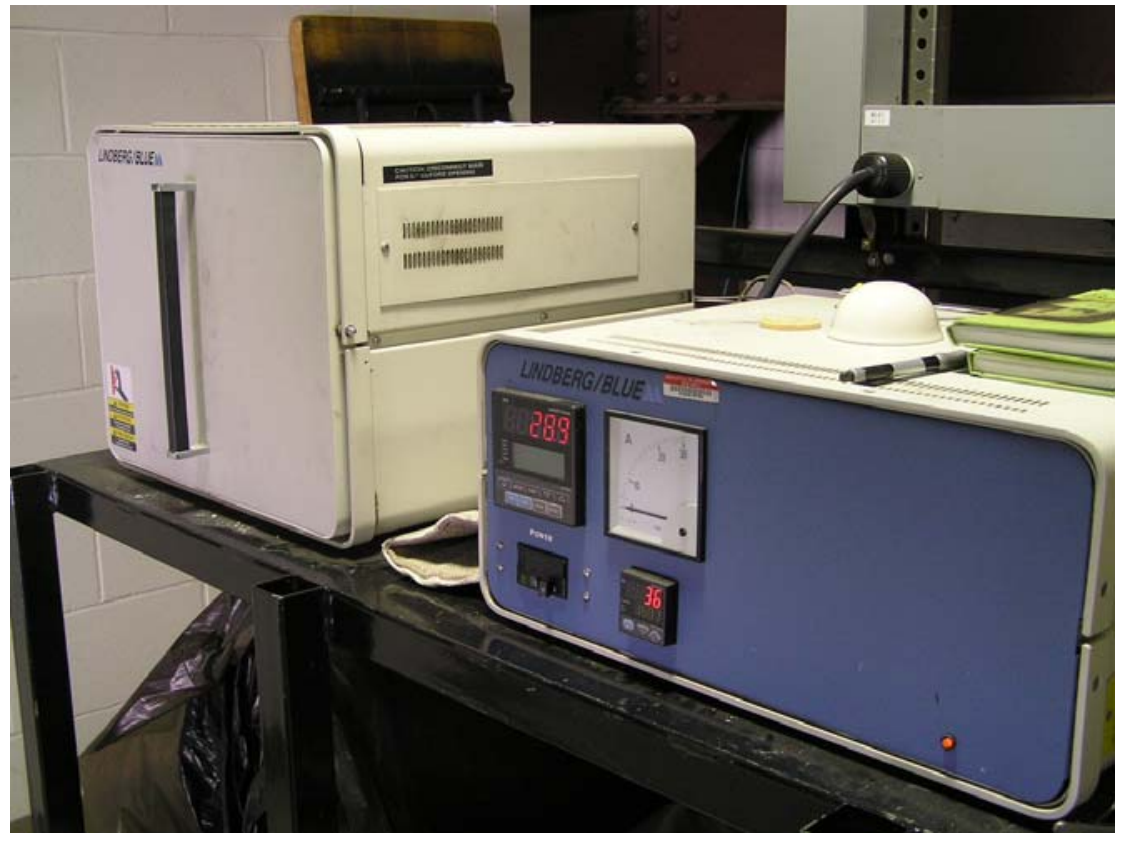

Figure 3.4 High temperature furnace

\subsection{Characterization analysis}

The morphology and composition of the ECR testing samples were analyzed using a JEOL JSM840A SEM equipped with a thermo electron EDS system. XRD pattern was gathered by a Panalytical X'Pert PRO diffractometer. 


\section{Research Objectives}

This research intends to accomplish the following objectives:

(1) Improve data analysis method for electrical conductivity relaxation technique to obtain reliable oxygen surface exchange and bulk diffusion coefficients. Study the fitted kinetic parameters relationship with experimental initial and final oxygen partial pressure.

(2) Investigate oxygen partial pressure effects on surface exchange and bulk diffusion coefficients obtained by electronic conductivity relaxation method for $\mathrm{La}_{0.6} \mathrm{Sr}_{0.4} \mathrm{Co}_{0.2} \mathrm{Fe}_{0.8} \mathrm{O}_{3-\delta}$.

(3) Develop the electronic conductivity relaxation method for infiltrated SOFC cathode. Measure the oxygen exchange coefficient at the interface of infiltrated material/LSCF.

(4) Establish a micro model for infiltrated SOFC cathode. Investigate the infiltrated material's specific role in oxygen reduction process and predict optimization of the infiltrated material based on the oxygen transport properties. 


\section{Data analysis method for relaxation experiments}

\subsection{Introduction}

As a useful and convenient tool for SOFCs cathode kinetic study, electrical conductivity relaxation $(E C R)$ technique attracted more interest recently. Researchers have investigated the effect of ECR testing conditions on the final fitted kinetic parameters. For instance, Otter derived equations which describe the transient response in conductivity relaxation experiments, taking into account the time needed for reactor flushing ${ }^{70}$. Wang et al. studied the effects of oxygen partial pressure step change together with the role of oxygen vacancy concentration on the relaxation kinetics ${ }^{71}$. Both instances show that careful control of the thermodynamic environment and proper mathematical treatment of the oxygen partial pressure step change will improve the quality of results.

Although the ECR technique has been widely used in various applications, details of the data fitting process have been rarely discussed. Most reports simply mention that a non-linear least square method was applied to obtain the fitted results. In this chapter, we will first analyze the problems of reference-reported ECR data fitting method. Improved ways will be offered in section 5.3 and further evaluation of the improved method is illustrated in section 5.4.

\subsection{Problems existed in reference reported method}

5.2.1 Determination of initial conductivity relaxation $\left(t_{0}\right)$

$$
\frac{\sigma(t)-\sigma(0)}{\sigma(\infty)-\sigma(0)}=1-\sum_{n=1}^{\infty} \frac{2 L^{2} \exp \left(-b_{n}^{2} D t / a^{2}\right)}{b_{n}^{2}\left(b_{n}^{2}+L^{2}+L\right)}
$$

As shown in equation 2.27 , the conductivity of the sample at the instant of first response to a step change in oxygen partial pressure must be known. In practice, the exact starting moment of 
conductivity relaxation is difficult to precisely detect due to the imperfect application of the $P_{\mathrm{O}_{2}}$ step change. Thus, equation 2.27 should be modified by introducing the actual starting response time $\left(t_{0}\right)$ as the third independent parameter, as shown in Equation 5.1.

$$
\frac{\sigma(t)-\sigma(0)}{\sigma(\infty)-\sigma(0)}=1-\sum_{n=1}^{\infty} \frac{2 L^{2} \exp \left[-b_{n}^{2} D\left(t-t_{0}\right) / a^{2}\right]}{b_{n}^{2}\left(b_{n}^{2}+L^{2}+L\right)}
$$

There are two primary methods to deal with $t_{0}$. The first method is to treat $t_{0}$ as an empirical parameter, just as with $D$ and $k^{49}$. The second method is to determine the value of $t_{0}$ using the physical reactor parameters (volume, gas flow rate) and an ideal flow model to calculate the reactor flush time ${ }^{67}$.

$$
\tau_{f}=\frac{V_{r}}{\phi_{v, t o t}} \cdot \frac{T_{S T P}}{T_{r}}
$$

$\phi_{v, \text { tot }}$ represents the total gas flow rate. $V_{r}$ and $T_{r}$ are the reactor's volume and temperature. And $T_{S T P}$ is the room temperature. The normalized oxygen partial pressure can be written as:

$$
\overline{P(t)}=\frac{P(t)-P(0)}{P(\infty)-P(0)}=1-\exp \left(-\frac{t}{\tau_{f}}\right)
$$

$t_{0}$ was fixed as $4 \tau_{f}$ in this study by considering that after a period of $4 \tau_{f}$, more than $98 \%$ of the original gas will be replaced, according to an ideal mixing model. To further improve the quality of fitted data, the superior method was selected by comparing the output of these two methods. 


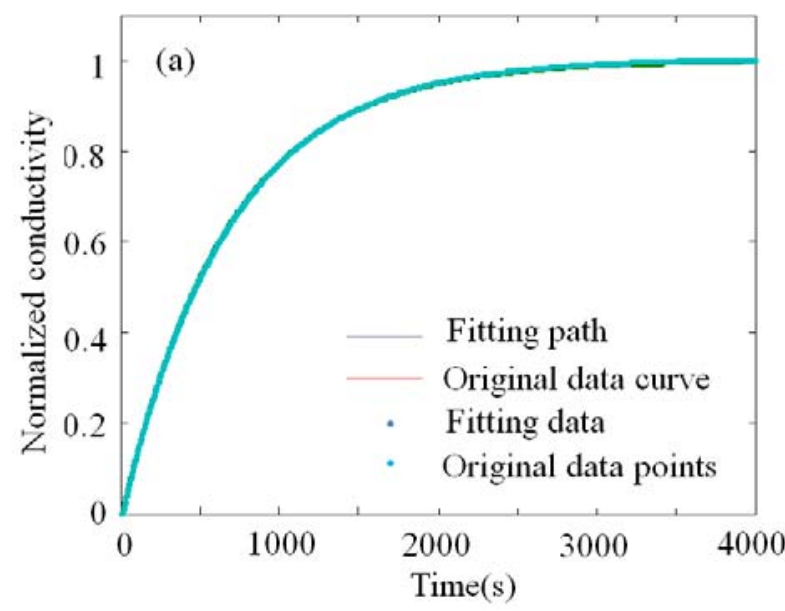

(a) Three parameters $\left(D, b_{1}, t_{0}\right)$

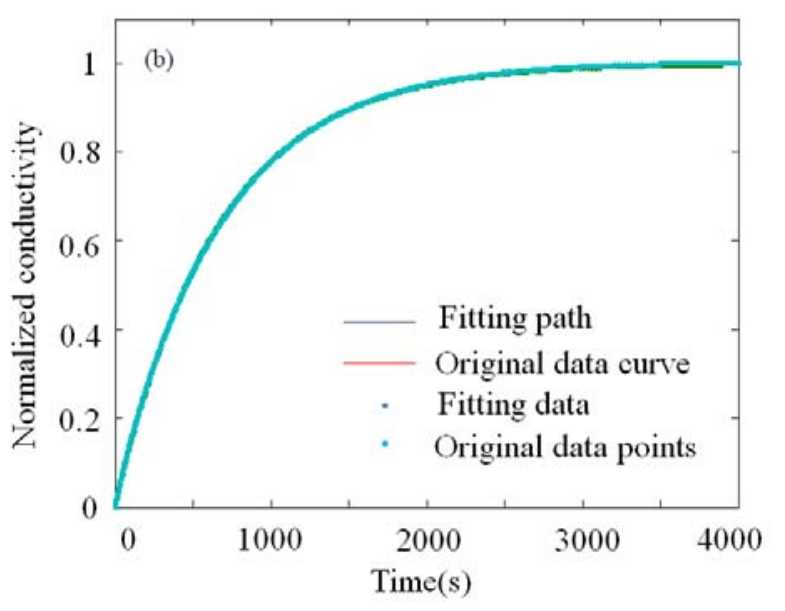

(b) Two parameters $\left(D, b_{1}\right)$

Figure 5.1 Depiction of fit quality between two methods for determining the starting relaxation time:

a) treating $t_{0}$ as an empirical parameter; and b) determining $t_{0}$ from reactor parameters and an ideally stirred tank model

A set of relaxation data was generated for fitting equation 5.1 using typical kinetic parameter values for MIEC and a time step of $2 \mathrm{~s}$. Parameter values were chosen as $\mathrm{D}=5 \times 10^{-6} \mathrm{~cm}^{2} / \mathrm{s}, \mathrm{k}=$ $1 \times 10^{-4} \mathrm{~cm} / \mathrm{s}, \mathrm{a}=0.05 \mathrm{~cm}, \mathrm{~b}_{1}=0.8603$, and $\mathrm{t}_{0}=14 \mathrm{~s}$. To fit Equation 5.1 directly, $b_{1}$ was applied using the selected value of $k$. The generated data were then fitted using the two methods for treating $\mathrm{t}_{0}$ using initial values for $k$ and $D$ that were equal to the values used for generating the relaxation data. Figure 5.1 depicts the resulting fitted curves that were obtained from MATLAB using the two different fitting schemes described. In the case in which $t_{0}$ was considered as a fitted parameter, the fitted results of $\left(D, b_{1}, t_{0}\right)$ are $\left(8.02 \times 10^{-6}, 0.68,11.94\right)$. In the case where the $t_{0}$ value is fixed as $14 \mathrm{~s}$, the fitted results of $\left(D, b_{1}\right)$ are $\left(5.79 \times 10^{-6}, 0.80\right)$. Comparison of the resulting values for $\left(D, b_{1}, t_{0}\right)$ to actual values showed that the method of treating $t_{0}$ as a fitting parameter caused greater error in the final predictions for all parameters. This single example 
here is illustrative of consistently superior results obtained in all tests by assignment of $t_{0}$ using mixing theory.

The superior result obtained from fixing $t_{0}$ is explained in terms of two primary problems with treating $t_{0}$ as a fitted parameter. First, all three of the fitted parameters $\left(D, b_{1}, t_{0}\right)$ are in the exponential term, but $t_{0}$ possesses the largest value and therefore dominates the term. Thus, fitted values of $D$ and $b_{1}$ are sensitive to minor fluctuation of the $t_{0}$ value during the fitting process, and numerous simulations show that fitted $D$ values may be obtained even with the same testing conditions. Second, the independent parameter method can not offer a physically convincing time zero for the ECR process, it only gives a best fit of the particular data set for a combination of $\left(D, b_{1}, t_{0}\right)$. Given these practical numerical problems, it is considered more reasonable to adopt an ideal physical model to fix $t_{0}$ than to treat it as a fitting parameter.

The effect of transient gas processes on the conductivity relaxation curve was ignored in this study, since the reactor flash time is usually very small compared to the total relaxation time (usually $<0.3 \%$ of the total relaxation time). However, if the reactor flush time can't be ignored the corrective diffusion model in den Otter's ${ }^{68}$ work should be applied.

\subsubsection{Determination of $D$ and $k$}

Song $^{72}$ et al. revealed their data analysis process for fitting the mixed controlled relaxation data. The detailed steps are illustrated as below. First, extreme cases were utilized to obtain the initial values of oxygen transport kinetic parameters. Equation 5.1 has two limiting versions, one each 
corresponding to surface exchange process dominant $\left(b_{l} \rightarrow 0\right)$ or bulk diffusion process dominant $\left(b_{1} \rightarrow \pi / 2\right)$.

$$
\begin{gathered}
\frac{\sigma(t)-\sigma(0)}{\sigma(\infty)-\sigma(0)}=1-\exp \left[-\frac{k\left(t-t_{0}\right)}{a}\right] \\
\frac{\sigma(t)-\sigma(0)}{\sigma(\infty)-\sigma(0)}=1-\frac{8}{\pi^{2}} \sum_{n=0}^{\infty} \frac{1}{(2 n+1)^{2}} \exp \left[-\frac{(2 n+1)^{2} \pi^{2} D\left(t-t_{0}\right)}{4 a^{2}}\right]
\end{gathered}
$$

Then calculate $\mathrm{L}$ value with the $k$ and $D$ values obtained by fitting equation 5.4 and 5.5.

$$
L=\frac{a}{l_{c}}=\frac{a k}{D}=b_{n} \tan b_{n}
$$

If $\mathrm{L}<1$, the relaxation process is surface exchange dominated and $k$ value is more precise than $D$. $\left(k, t_{0}\right)$, which was obtained by fitting equation 5.4 , will be applied as initial values for fitting equation 5.1 ( $D$ can be presented by $a, k$ and $b_{1}$ using equation 2.28). Similarly, if $\mathrm{L}>1,\left(D, t_{0}\right)$ will be used as the initial fitting values. For both cases, $b_{1}$ was chosen as $\mathrm{L}=1$.

However, the simultaneously fitted $D$ and $k$ values using Song's method was found not reliable in this research. Besides, Cox-Galhotra ${ }^{69}$ et al. also addressed the low reliability of the fitting procedure that seeks to simultaneously determine $D$ and $k$. Sample properties such as grain size and surface roughness that impacted results had been investigated. The authors suggested testing samples under a single process controlled region to reduce the number of adjustable parameters and obtain more reliable fitted results. The problem of analyzing a single relaxation data set is described in the following part.

In the first analysis, fitting error in $\left(D, b_{1}\right)$ plane is explored. Generated relaxation data with parameter values of $\left(D=5 \times 10^{-6} \mathrm{~cm}^{2} / \mathrm{s}, b_{l}=0.8603, t_{0}=0\right)$ were analyzed. To focus on analyzing the 
fitting process, we ignore $t_{0}$ by setting its value to 0 in the rest part of this chapter. As shown in the color maps of Figure 5.2, several local minima were located in the mixed control region where $b_{1}$ varies from 0.3 to 1.4 . Since the global minimum is smaller than all other minima, it is possible to distinguish the global minimum through the non-linear least square fitting process, but the algorithm requires an appropriate starting point. In other words, it is possible to fit exact parameter values by analyzing a single ideal relaxation data set, but only if a sufficiently accurate initial guess is made and the relaxation data are noise free.

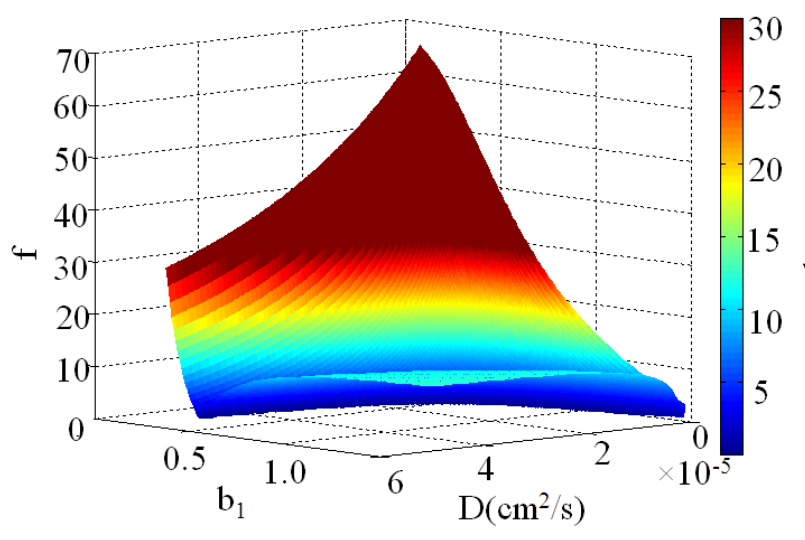

(a)

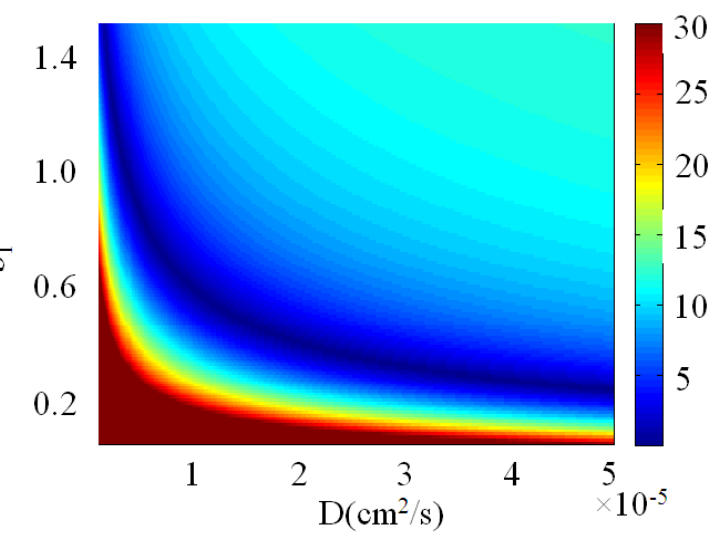

(b)

Figure 5.2 Error color map for fitting single relaxation data

Unfortunately, perfect relaxation data can not be obtained in practice due to random variations (noise) in the measured signals. To test the fitting quality for a noisy single data set, the MATLAB function "randn" is used to superimpose a $+/-2 \%$ noise signal on the base data. As shown in the error map of Figure 5.3, extraction of the global minimum is difficult with noisy data because the values of local minimums are near each other. The result implies that the 
accuracy of the fitted parameters determined from a single data set possesses ever increasing uncertainty as practical data are applied.

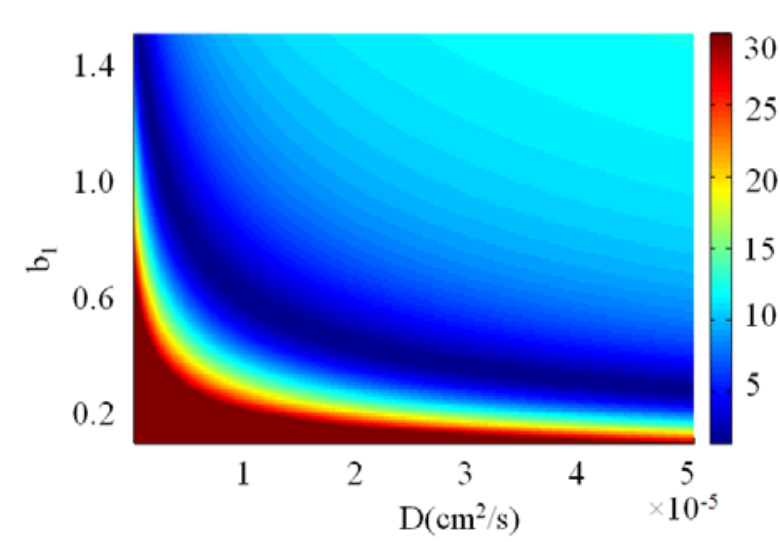

(a)

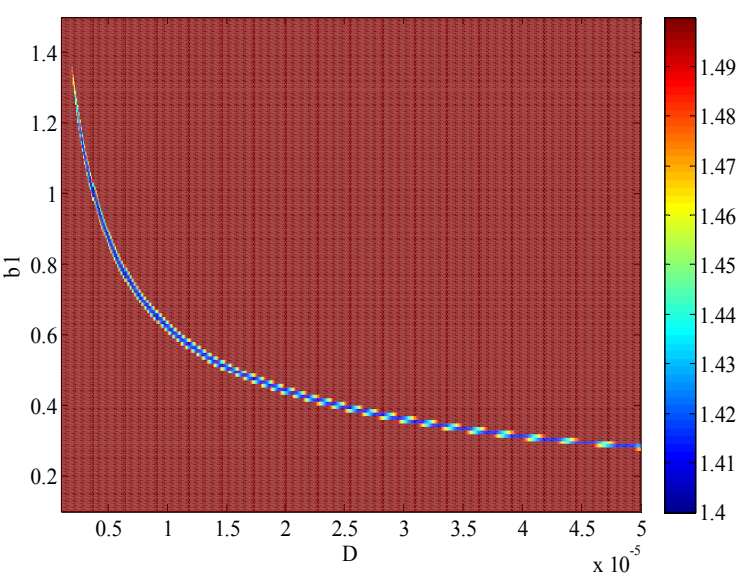

(b)

Figure 5.3 Error color map for fitting single noisy relaxation data

Error map for wide range of $k$ and $D$;

(b) Error map with adjusted scale showing error tolerance band of 5\%

To further verify the above conclusion, two different initial combinations of values were applied to fit the noisy relaxation data. In the first description the $\left(D, b_{1}\right)$ values of $\left(5 \times 10^{-6}, 0.8603\right)$ are used to generate the fitted results of $\left(7.04 \times 10^{-6}, 0.7394\right)$. Then $\left(D, b_{1}\right)$ initial values of $\left(3 \times 10^{-5}\right.$, $0.6000)$ were utilized. The corresponding fitted results are $\left(1.06 \times 10^{-5}, 0.6048\right)$, and the fitting curves and original data points are shown in Figure 5.4. With the different $\left(D, b_{1}\right)$ values, both of the fitting curves qualitatively fitted the original data points well, but significant errors appeared in the fitted prediction for the global minimum. This further proves that the kinetic parameters obtained by analyzing a single relaxation data set are unreliable even though qualitatively good fitting curves can be generated. 

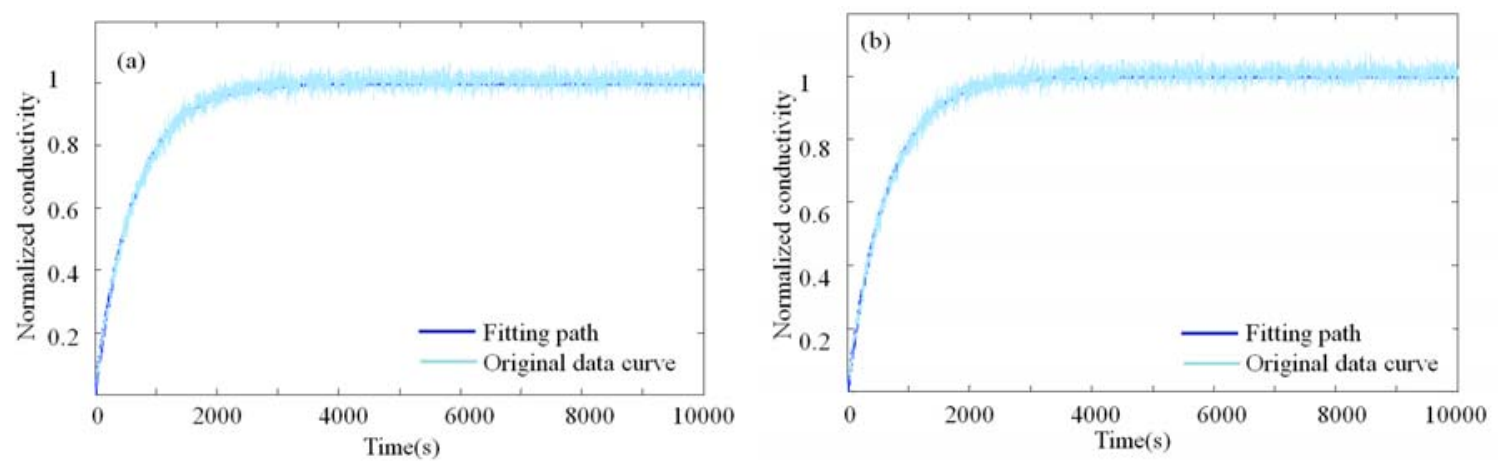

Figure 5.4 Fitting curve for single relaxation data with different initials

(a) $D=5 \times 10^{-6} \mathrm{~cm}^{2} / \mathrm{s}, b_{I}=0.8603$ (b) $D=3 \times 10^{-5} \mathrm{~cm}^{2} / \mathrm{s}, b_{I}=0.6000$

According to the above analysis, there are two possible problems using the data fitting process reported by Song. First, the accuracy of initial values can not be evaluated in a case where a single control process is assumed, but a mixed control process actually exists. Second and perhaps more importantly, a global minimum of the error may not be obtained, depending on the initial guess of $D$ and $k$ used in the fitting process. If the initial values used are not close enough to the real values, the calculated minimum may only be a local minimum and therefore erroneous.

\subsection{Improvement of data analysis method}

In order to improve the accuracy of parameters assigned through analysis of ECR test data and to compensate for signal noise, a method is proposed that utilizes two data sets in the fitting process. Relaxation data are generated for two samples with different assumed thicknesses, but operated under identical conditions. In other words, $(D, k)$ values are considered constant for both generated relaxation data sets but the ' $a$ ' value is adjusted to impose a variation in the dominant oxygen transport control mechanism. Each data set is fitted for $D$ and $k$ (since $b_{1}$ varies with $a$ ) 
and the fitting errors are determined for each set. The errors for each set are then compared using a weighted mean average error method to assign the optimized parameter intersection to all of the relaxation data.

$$
f=\sqrt{\frac{F_{1}^{2}}{T_{1}}+\frac{F_{2}^{2}}{T_{2}}}
$$

$F_{1}$ and $F_{2}$ are the fitting error vectors separately for the two data sets. $T_{1}$ and $T_{2}$ represent the data length. $\frac{F_{1}}{\sqrt{T_{1}}}+\frac{F_{2}}{\sqrt{T_{2}}}$ is the weight average error function. And $f$ is the quantified value by using norm command in MATLAB. A +/-2\% noise signal was superimposed on all the generated relaxation data in the following analyses.

Table 5.1 Parameter values assumed to generate conductivity relaxation data for cases of mixed control

$$
\left(\mathrm{L}_{1}=1\right) \text { and bulk diffusion control }\left(\mathrm{L}_{2}=10\right)
$$

\begin{tabular}{c|c|c|c|c|c}
\hline & $\begin{array}{c}D \\
{\left[\mathrm{~cm}^{2} / \mathrm{s}\right]}\end{array}$ & $\begin{array}{c}k \\
{[\mathrm{~cm} / \mathrm{s}]}\end{array}$ & $\begin{array}{c}b_{1} \\
{[-]}\end{array}$ & $\begin{array}{c}a \\
{[\mathrm{~cm}]}\end{array}$ & $\begin{array}{c}\mathrm{L} \\
{[-]}\end{array}$ \\
\hline 1 & $5 \times 10^{-6}$ & $1 \times 10^{-4}$ & 0.8603 & 0.05 & 1 \\
\hline 2 & $5 \times 10^{-6}$ & $1 \times 10^{-4}$ & 1.4289 & 0.50 & 10 \\
\hline
\end{tabular}

The limiting cases of single dominant control mechanisms are considered first. In the first case, dominant bulk diffusion control is compared to mixed control using the parameter values listed in Table 5.1. Relaxation data were generated assuming mixed control $\left(\mathrm{L}_{1}=1\right)$ using data set 1 parameters and bulk diffusion dominance $\left(\mathrm{L}_{2}=10\right)$ using data set 2 parameters. A fitting error color map for data set 1 in the $D, k$ plane is shown in Figure 5.5. As when fitting with $\left(D, b_{1}\right)$, 
several close local error minima were observed in the fitting results when using the single data set. By adjusting the scale of the figure, a 5\% tolerance band can be determined around the values of $k$ and $D$ producing the global minimum error. Figure 5.5(b) shows that a more accurate oxygen surface exchange coefficient can be obtained compared to the bulk diffusion coefficient.

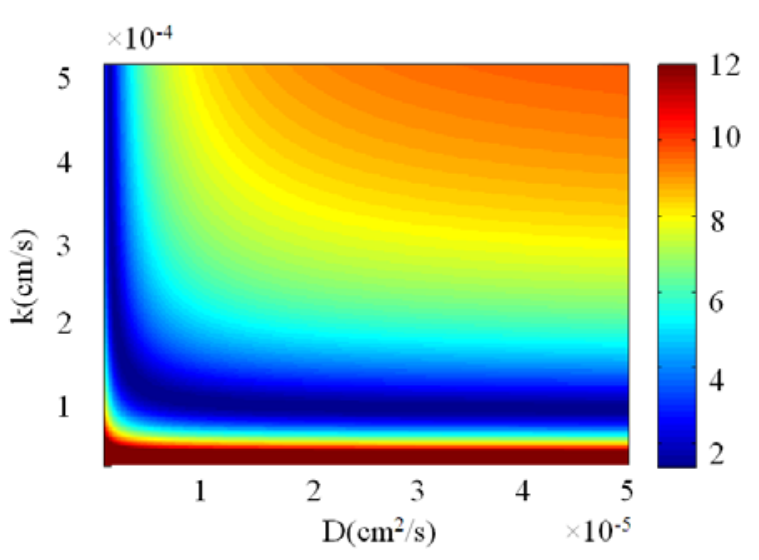

(a)

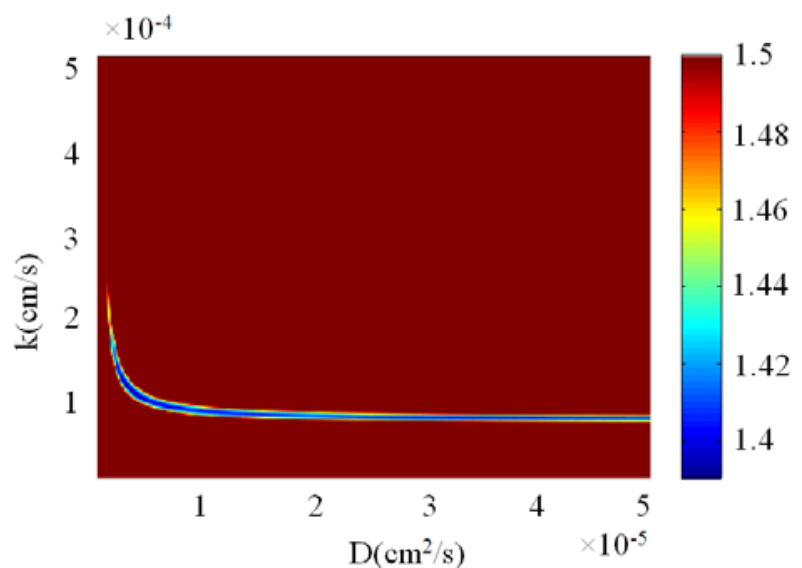

(b)

Figure 5.5 Error color map for single relaxation data fitting

(a) Error map for wide range of $k$ and $D$;

(b) Error map with adjusted scale showing error tolerance band of 5\%

To explain this phenomenon, kinetic parameters effects on the relaxation process will be discussed. Assuming L is either close to 0 or infinity, two limiting versions of equation 2.27 can be obtained. As shown in equation 5.4 and 5.5, those limitations are the diffusion equation solutions for single step control.

Based on these two limitations, relaxation curves were generated either under oxygen surface exchange or bulk diffusion control $(\mathrm{a}=0.05 \mathrm{~cm})$, as shown in Figure 5.6. Possible $k$ and $D$ values 
of real materials were chosen to generate the relaxation curves. Results showed that relaxation time varied more under diffusion control than surface exchange control. It indicated the uncertainty of $D$ is higher than $k$ for the mixed control relaxation data fitting.
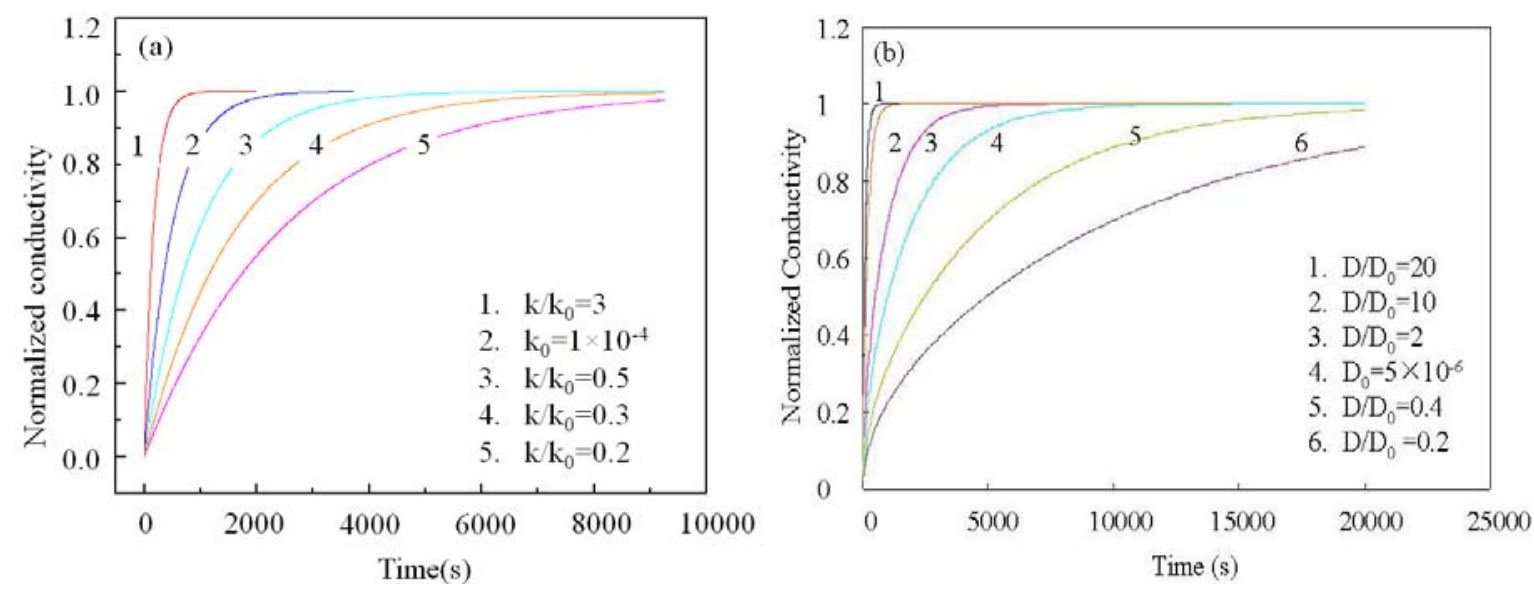

Figure 5.6 Kinetic parameters effects on electrical conductivity relaxation time

Figure 5.7 shows the fitting error color maps in the $D, k$ plane for simultaneous analysis of the two data sets obtained on samples with different thicknesses. Unlike the analysis result for the single relaxation data sets, the objective function (error) possesses a single minimum. Comparing the Figures $5.5 \mathrm{~b}$ and $5.7 \mathrm{~b}$, the estimation error for oxygen surface exchange coefficient was decreased by $80 \%$ within a $5 \%$ variation of minimum error. The range of fitted oxygen bulk diffusion coefficient values was narrowed to between $4.7 \sim 5.7 \times 10^{-6} \mathrm{~cm}^{2} / \mathrm{s}$. Therefore, reliable oxygen kinetic parameter values can be assigned accurately with a small tolerance by testing two different sample thicknesses at the same condition and simultaneously fitting results. 


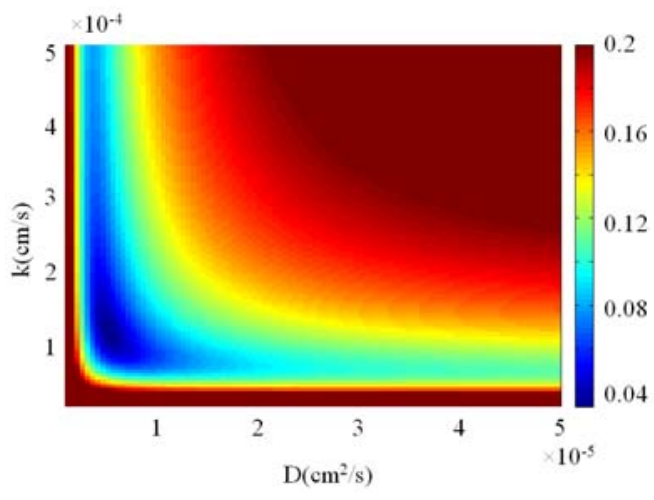

(a)

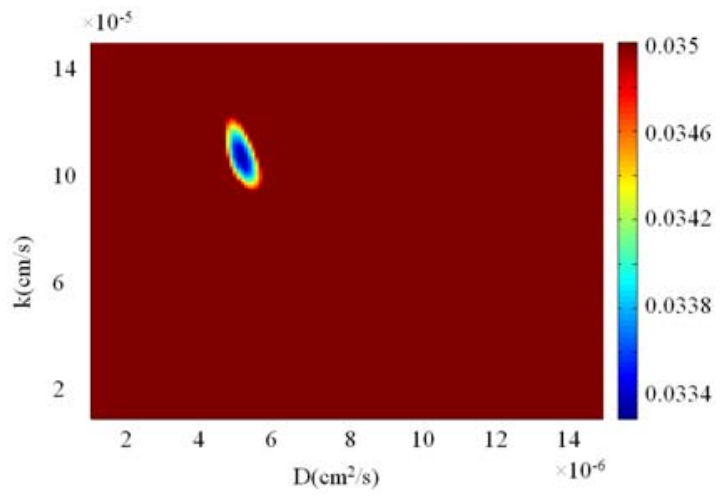

(b)

Figure 5.7 Error color map for ECR fitting $\left(\mathrm{L}_{1}=1, \mathrm{~L}_{2}=10\right)$

(a) Error map for wide range of $k$ and $D$;

(b) Error map with adjusted scale showing error tolerance band of 5\%

A similar analysis can be performed to test the accuracy of parameter predictions while assuming that relaxation processes are under surface exchange control $\left(\mathrm{L}_{2}=0.1\right)$. In this case, data are generated using the parameters shown in Table 5.2, first for the case of mixed control $\left(\mathrm{L}_{1}=1\right)$ and then for the case of surface limited control $\left(\mathrm{L}_{2}=0.1\right)$. The fitted result is shown in Figure 5.8, and only one minimum is obtained in the fitting error map.

The fitting results obtained in these two extreme cases suggest the best sample thicknesses to use in ECR testing. Since the uncertainty in the bulk diffusion coefficient increased when using thin samples, thicker samples will be considered in practice. However the sample thickness is limited by assumptions of the applied methods and practical limitations. The Van der Pauw method ${ }^{73}$ is applicable for thin sheets, and has been used widely to measure sample conductivity in ECR testing. Therefore, it is not practical to test a very thick sample $(\mathrm{L}=10)$. Based on the present analysis, two sample thicknesses are used to verify accuracy in a real system. 
Table 5.2 Parameters values of generated conductivity relaxation data $\left(\mathrm{L}_{1}=1, \mathrm{~L}_{2}=0.1\right)$

\begin{tabular}{c|c|c|c|c|c}
\hline Parameters & $\begin{array}{c}D \\
{\left[\mathrm{~cm}^{2} / \mathrm{s}\right]}\end{array}$ & $\begin{array}{c}k \\
{[\mathrm{~cm} / \mathrm{s}]}\end{array}$ & $\begin{array}{c}b_{1} \\
{[-]}\end{array}$ & $\begin{array}{c}a \\
{[\mathrm{~cm}]}\end{array}$ & $\begin{array}{c}\mathrm{L} \\
{[-]}\end{array}$ \\
\hline 1 & $5 \times 10^{-6}$ & $1 \times 10^{-4}$ & 0.8603 & 0.05 & 1 \\
\hline 2 & $5 \times 10^{-6}$ & $1 \times 10^{-4}$ & 0.3111 & 0.005 & 0.1 \\
\hline
\end{tabular}

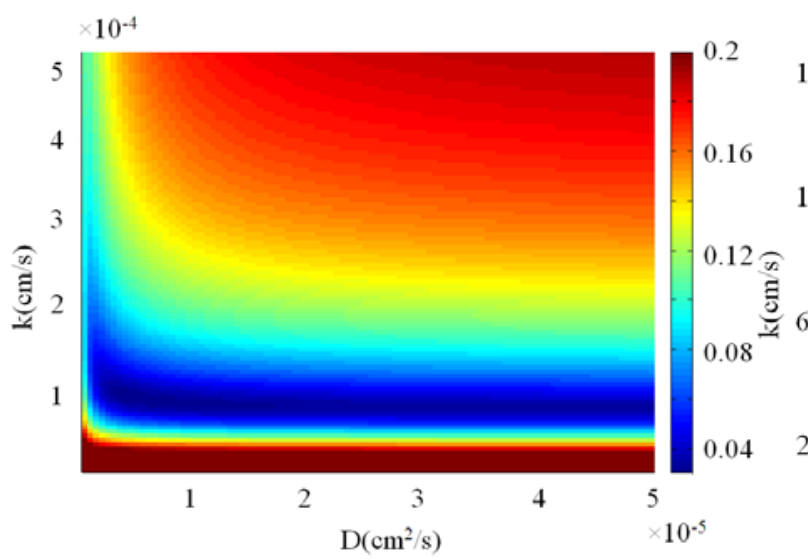

(a)

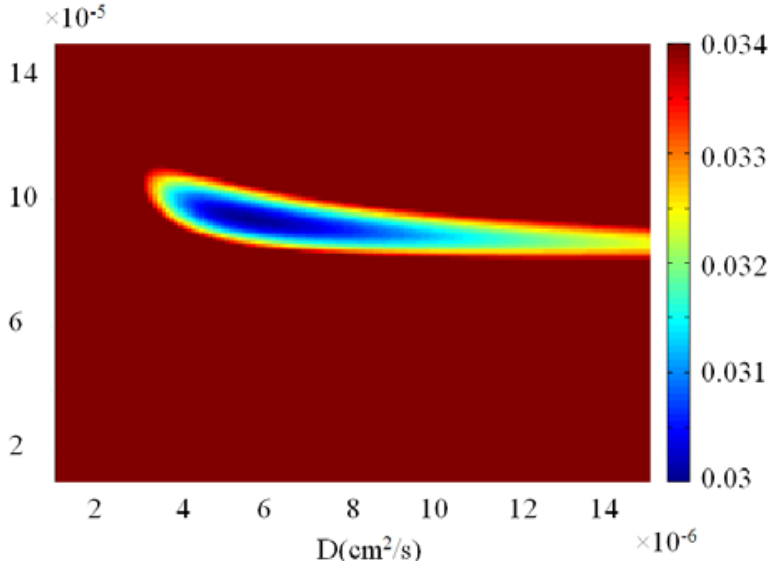

(b)

Figure 5.8 Error color map for fitting two relaxation data sets $\left(\mathrm{L}_{1}=1, \mathrm{~L}_{2}=0.1\right)$

(a) Error map for wide range of $k$ and $D$;

(b) Error map with adjusted scale showing error tolerance band of 5\%

Thicknesses $a_{1}=0.05 \mathrm{~cm}\left(\mathrm{~L}_{1}=1\right)$ and $a_{2}=0.15 \mathrm{~cm}\left(\mathrm{~L}_{2}=3\right)$ were chosen for generating ECR data sets. All the other parameters are listed in Table 5.3. The fitting error color map shown in Figure 5.9 indicates that reliable $D$ and $k$ values can be obtained by testing the two practical sample thicknesses, one of which is thick enough to accentuate bulk diffusion limitations. 
Table 5.3 Parameters values of generated conductivity relaxation data

\begin{tabular}{c|c|c|c|c|c}
\hline & $\begin{array}{c}D \\
\left(\mathrm{~cm}^{2} / \mathrm{s}\right)\end{array}$ & $\begin{array}{c}k \\
(\mathrm{~cm} / \mathrm{s})\end{array}$ & $b_{1}$ & $\begin{array}{c}a \\
(\mathrm{~cm})\end{array}$ & \\
\hline 1 & $5 \times 10^{-6}$ & $1 \times 10^{-4}$ & 0.8603 & 0.05 & 1 \\
\hline 2 & $5 \times 10^{-6}$ & $1 \times 10^{-4}$ & 1.1925 & 0.15 & 3 \\
\hline
\end{tabular}

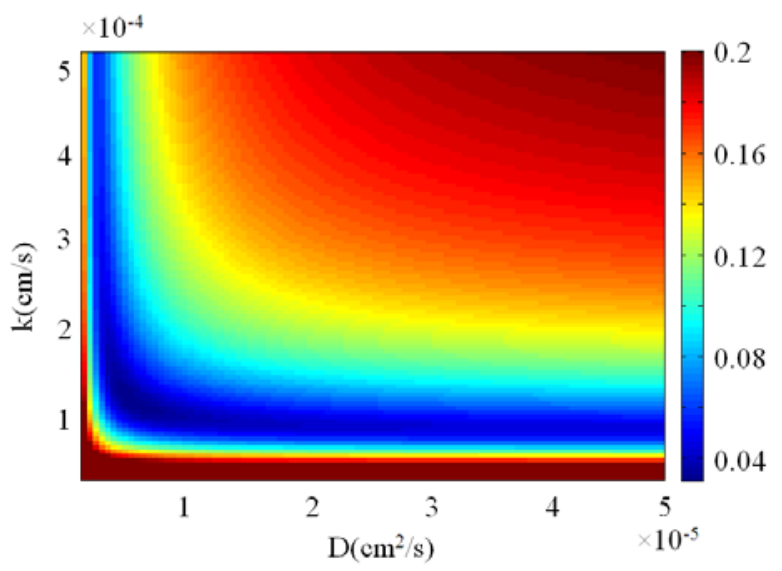

(a)

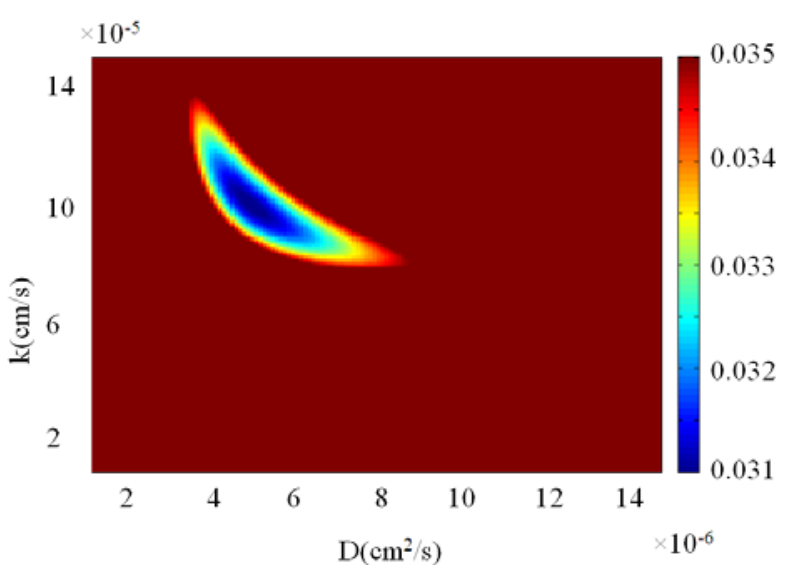

(b)

Figure 5.9 Error color map for fitting two noisy relaxation data sets $\left(\mathrm{L}_{1}=1, \mathrm{~L}_{2}=3\right)$

(a) Error map for wide range of $k$ and $D$;

(b) Error map with adjusted scale showing error tolerance band of 5\%

To check whether analyzing more relaxation data sets can improve the fitted results' accuracy, three generated data sets were analyzed. The parameters used for generation of relaxation data are listed in table 5.4. Practical sample thicknesses were applied. The fitting error color map for these three relaxation data sets is presented in Figure 5.10. A significant improvement in fitting 
quality was not detected by using the three relaxation data sets compared to using two data sets.

Hence, more relaxation data sets involved in the fitting process will not be considered.

Table 5.4 Parameters values of generated conductivity relaxation data

\begin{tabular}{c|c|c|c|c|c}
\hline Parameters & $\begin{array}{c}D \\
{\left[\mathrm{~cm}^{2} / \mathrm{s}\right]}\end{array}$ & $\begin{array}{c}k \\
{[\mathrm{~cm} / \mathrm{s}]}\end{array}$ & $\begin{array}{c}b_{1} \\
{[-]}\end{array}$ & $\begin{array}{c}a \\
{[\mathrm{~cm}]}\end{array}$ & $\begin{array}{c}\mathrm{L} \\
{[-]}\end{array}$ \\
\hline 1 & $5 \times 10^{-6}$ & $1 \times 10^{-4}$ & 0.8603 & 0.05 & 1 \\
\hline 2 & $5 \times 10^{-6}$ & $1 \times 10^{-4}$ & 0.3111 & 0.005 & 0.6 \\
\hline 3 & $5 \times 10^{-6}$ & $1 \times 10^{-4}$ & 1.1925 & 0.15 & 3 \\
\hline
\end{tabular}

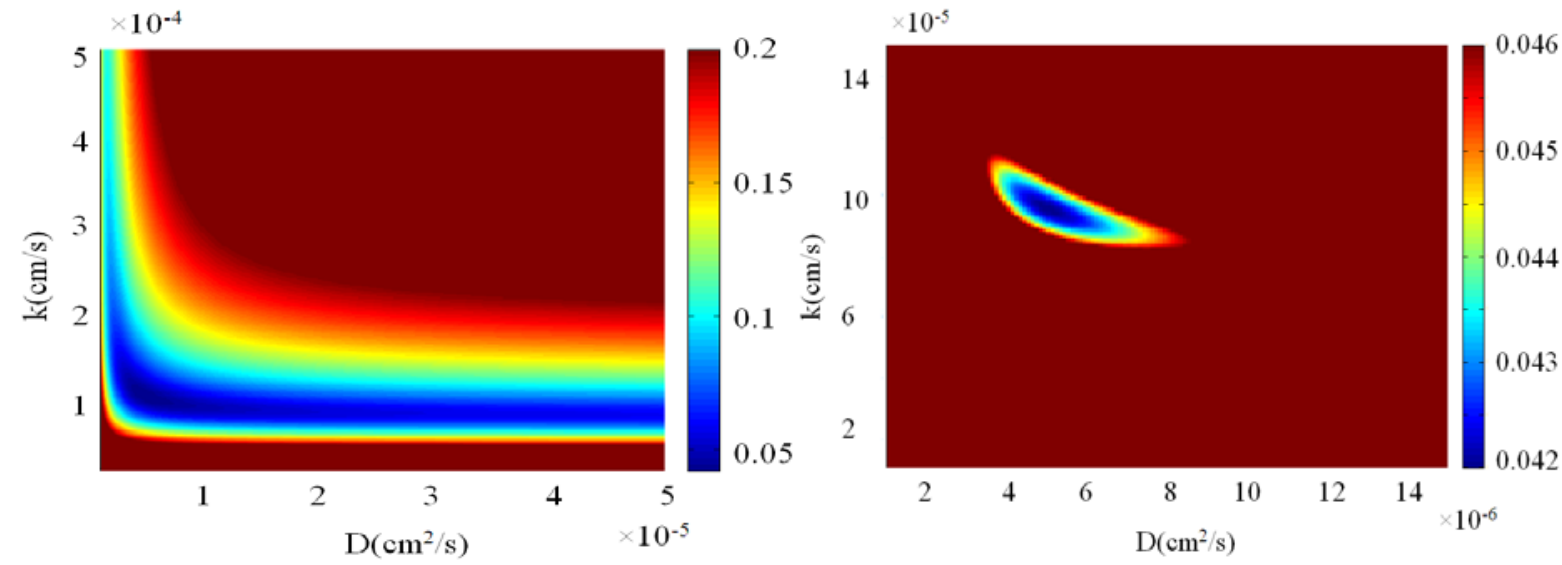

Figure 5.10 Error color map for fitting three relaxation data sets $\left(\mathrm{L}_{1}=1, \mathrm{~L}_{2}=0.6, \mathrm{~L}_{3}=3\right)$

(a) Error map for wide range of $k$ and $D$;

(b) Error map with adjusted scale showing error tolerance band of 5\% 


\subsection{Concluding Remarks:}

The process of analyzing electrical conductivity relaxation data was discussed in this chapter. The practice of fitting a single data set in existing methods has been shown to be deficient as it produces inaccurate values for the fitted parameters. Further it was shown that when applying a noise signal comparable to that typical of experiments, qualitative fits to the data are acceptable while quantitative fits are widely variable. These observations arising from simulations have practical implications for real experiments and data analysis, and indicate that improvements are required to enhance the predictive accuracy of the ECR data fitting techniques.

Two primary alterations to conventional models are described here. First, comparisons are made between two different methods to determine actual time zero $\left(t_{0}\right)$. The results showed that conventional methods of treating $\mathrm{t}_{0}$ as a fitting parameter introduce inaccuracy to the parameter evaluation. Adoption of a continuously ideally stirred tank reactor model to fix the actual time zero before fitting relaxation data improved the reliability of the fitted results. Second, enhanced accuracy of $D$ and $k$ are obtained by fitting two sets of data and plotting the error intersection. The two data sets should be obtained for sample thicknesses of $\mathrm{L}=1$ and $\mathrm{L}=3$. It was further shown that data fitting using three data sets did not substantially improve the quality of the fit.

By adopting the suggested improvements to the ECR testing and data analysis process, more accurate predictions for $\mathrm{k}$ and $\mathrm{D}$ may be obtained than are currently available. Values for $\mathrm{k}$ and D can be determined with approximately $+/-30 \%$ accuracy in a $5 \%$ precision band, which compares favorably with conventional methods that typically produce greater than $+/-100 \%+$ accuracy in a $5 \%$ precision band and often cannot identify globally accurate results. The 
improved accuracy of the enhanced method allows greater correlation of thermodynamic conditions with measured oxygen transport coefficients. 


\section{Chemical diffusion and oxygen surface exchange of $\mathrm{La}_{0.6} \mathrm{Sr}_{0.4} \mathrm{Co}_{0.2} \mathrm{Fe}_{0.8} \mathrm{O}_{3-\delta}$ studied with electrical conductivity relaxation}

\subsection{Introduction}

Perovskite-type oxides such as $\mathrm{La}_{1-\mathrm{x}} \mathrm{Sr}_{\mathrm{x}} \mathrm{Co}_{1-\mathrm{y}} \mathrm{Fe}_{\mathrm{y}} \mathrm{O}_{3-\delta}$ (LSCF) possess mixed ionic and electronic conductivity. They also display good mechanical and chemical stability compared to some other mixed conducting oxides. For these reasons, LSCF has attracted wide commercial interest for use as the SOFC cathode.

In this chapter, oxygen transport behavior of $\mathrm{La}_{0.6} \mathrm{Sr}_{0.4} \mathrm{Co}_{0.2} \mathrm{Fe}_{0.8} \mathrm{O}_{3-\delta}$ has been investigated with oxygen partial pressure varying from $0.02 \mathrm{~atm}$ to $0.20 \mathrm{~atm}$. The improved ECR data analysis method introduced in Chapter 5 was applied to obtain oxygen surface exchange and bulk diffusion coefficients. The result trend displayed is that both of the parameters decreased with decreasing oxygen partial pressure. With analysis of the oxygen transport process, the correlation among oxygen surface exchange coefficient, oxygen reduction reaction constants and oxygen partial pressure was revealed.

\subsection{LSCF pellet preparation and characterization}

The experimental samples used in this study were made with commercial $\mathrm{La}_{0.6} \mathrm{Sr}_{0.4} \mathrm{Co}_{0.2} \mathrm{Fe}_{0.8} \mathrm{O}_{3-\delta}$ powder (NexTech Materials, Ltd.). Using a 19mm (diameter) die, the powders were axially pressed at $80 \mathrm{MPa}$ and sintered at $1350^{\circ} \mathrm{C}$ in air for $2 \mathrm{~h}$, resulting in a plane disk shape with a diameter of $15.4 \mathrm{~mm}$. Then, LSCF pellets were progressively polished on both sides using silicon carbide polishing papers followed by polycrystalline diamond suspension up to $1 \mu \mathrm{m}$ (PACE Technologies, Tucson, Arizona, USA). After polishing, LSCF sample thicknesses are $1.5 \mathrm{~mm}$ and 
$2.7 \mathrm{~mm}$. All sintered samples were confirmed as in excess of $97 \%$ theoretical density using the Archimedes method (AD-1653 specific gravity measuring kit, A\&D Company).

Figure 6.1 shows the surface morphologies and chemical composition of the LSCF sample after sintering. Fine grain can be clearly seen from the morphology yet the grain size distribution is not even. Average grain size is around $3 \mu \mathrm{m}$. Surface chemical composition results obtained from EDS indicate that the element stoichiometry is $\mathrm{La}_{0.64} \mathrm{Sr}_{0.38} \mathrm{Co}_{0.2} \mathrm{Fe}_{0.76} \mathrm{O}_{3-\delta}$ which compares favorably by the theoretical stoichiometry of $\mathrm{La}_{0.6} \mathrm{Sr}_{0.4} \mathrm{Co}_{0.2} \mathrm{Fe}_{0.8} \mathrm{O}_{3-\delta}$. After polishing, grain boundaries were not detected by SEM but some internal pores were observed. XRD patterns of the LSCF sintered pellet and the commercial LSCF powder are given in Figure 6.2 and verify phase stability during sintering.

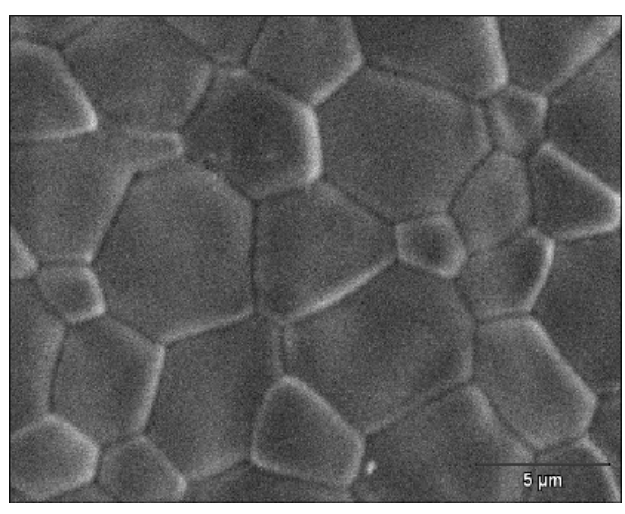

(a)

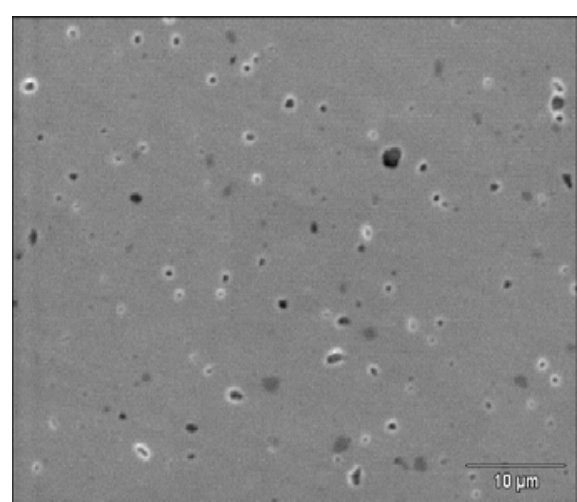

(b)

\begin{tabular}{lllll}
\hline & La & Sr & Co & Fe \\
& & & & \\
\hline at. $\%$ & 32.3 & 19.4 & 10.4 & 37.9 \\
\hline
\end{tabular}

Figure 6.1 Surface morphologies and surface chemical composition of sintered LSCF pellet

(a) Before polishing (b) After polishing 


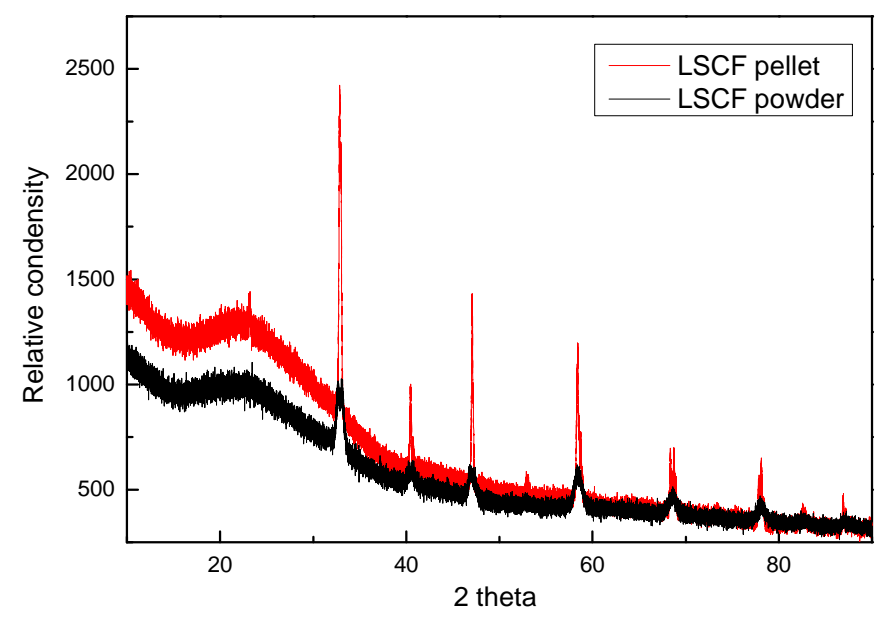

Figure 6.2 XRD patterns for commercial LSCF powder and sintered LSCF pellet

\subsection{LSCF defects and mixed conductivity}

Using Kröger-Vink notation, the intrinsic defect chemistry of $\mathrm{La}_{0.6} \mathrm{Sr}_{0.4} \mathrm{Co}_{0.2} \mathrm{Fe}_{0.8} \mathrm{O}_{3-\delta}$ can be described by the following defect equilibrium:

$$
\begin{aligned}
& n u l l \rightarrow V_{L a}^{\prime \prime \prime}+V_{B}^{\prime \prime \prime}+3 V_{O}^{*} \\
& n u l l \rightarrow e^{\prime}+h \\
& 2 B_{B}^{x} \rightarrow B_{B}^{\prime}+B_{B}
\end{aligned}
$$

$B$ represents Co and Fe. Oxygen ion transport is generally considered to occur via the vacancy hopping mechanism. On the other hand, the electronic conductivity of LSCF is a result of different $B$ site chemical status. The oxidation $(\delta>0)$, reduction status and acceptor incorporation of LSCF can be described as Equation 6.4-6.7.

$$
\begin{aligned}
\frac{3}{2} O_{2}+6 B_{B}^{x} & \rightarrow 3 O_{O}^{x}+V_{L a}^{\prime \prime \prime}+V_{B}^{\prime \prime \prime}+6 B_{B}^{\cdot} \\
O_{O}^{x} & \rightarrow \frac{1}{2} O_{2}+V_{O}^{\prime \prime}+2 e^{\prime}
\end{aligned}
$$




$$
\begin{aligned}
2 \mathrm{SrO}+1 / 2 \mathrm{O}_{2}+2 \mathrm{~B}_{B}^{x} & \rightarrow 2 \mathrm{Sr}_{L a}^{\prime}+3 \mathrm{O}_{O}^{x}+2 B_{B} \\
2 \mathrm{SrO} & \rightarrow 2 \mathrm{Sr}_{L a}^{\prime}+2 \mathrm{O}_{O}^{x}+V_{O}^{*}
\end{aligned}
$$

Due to electro-neutrality and site conservation, the following equations can be derived

$$
\begin{aligned}
& 3\left[V_{L a}^{\prime \prime \prime}\right]+3\left[V_{B}^{\prime \prime \prime}\right]+\left[S r_{A}^{\prime}\right]+\left[B_{B}^{\prime}\right]=2\left[V_{O}^{\bullet}\right]+\left[B_{B}^{\cdot}\right] \\
& {\left[B_{B}^{\prime}\right]+\left[B_{B}^{x}\right]+\left[B_{B}^{\prime}\right]=1} \\
& {\left[O_{O}^{x}\right]+\left[V_{o}^{\ddot{c}}\right]=3}
\end{aligned}
$$

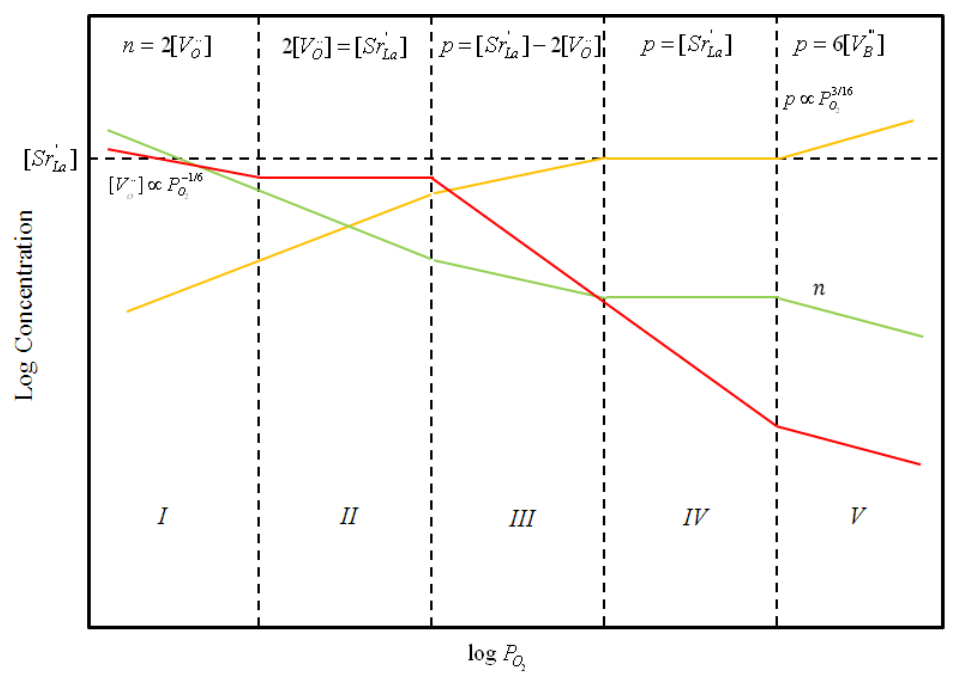

Figure 6.3 Schematic representation of concentration of oxygen vacancy and electronic defects in LSCF as a function of oxygen partial pressure

The Brouwer diagram for LSCF was obtained by further analyzing defect concentration variation with oxygen partial pressure, as shown in Figure 6.3. Concentrations of the main defects are plotted on a logarithmic scale against the logarithm of the oxygen partial pressure. It can be seen that there are five regions based on the dominant defect type. Region $I$ represents the reduced status at low $P_{O_{2}}$. The main defect chemistry can be described by Equation 6.5. Corresponding equilibrium constant is given by: 


$$
K_{r}=\frac{P_{O_{2}}^{1 / 2} n^{2}\left[V_{o}^{\cdot \cdot}\right]}{\left[O_{O}^{x}\right]}
$$

Since the concentration of oxygen ions in the crystal is not significantly changed and the concentration of the electronic carrier equals twice that of the vacancy concentration, a relation between oxygen vacancy concentration and $P_{O_{2}}$ can be deduced:

$$
\left[V_{o}^{\cdot \cdot}\right] \propto P_{O_{2}}^{-1 / 6}
$$

Similarly, in region $V$, the main defects are the metal vacancies and the defect chemistry can be described as Equation 6.4. The equilibrium constant can be written as:

$$
K_{o}=\frac{\left[O_{O}^{x}\right]^{3}\left[V_{L a}^{\prime \prime \prime}\right]\left[V_{B}^{\prime \prime \prime}\right]\left[B_{B}\right]^{6}}{P_{O_{2}}^{3 / 2}\left[B_{B}^{x}\right]^{6}}
$$

So the oxygen partial pressure dependence for electronic hole carrier is:

$$
p \propto P_{O_{2}}^{3 / 16}
$$

Besides, with doping strontium, LSCF is a p-type semiconductor in region $I I I, I V$ and $V$.

Mantzavinos $^{74}$ et al. studied LSCF oxygen non-stoichiometry under different oxygen partial pressure, as shown in Figure 6.4. It can be roughly estimated that in our testing environment $\delta$ value is approximately between 0.02 and 0.03 . 


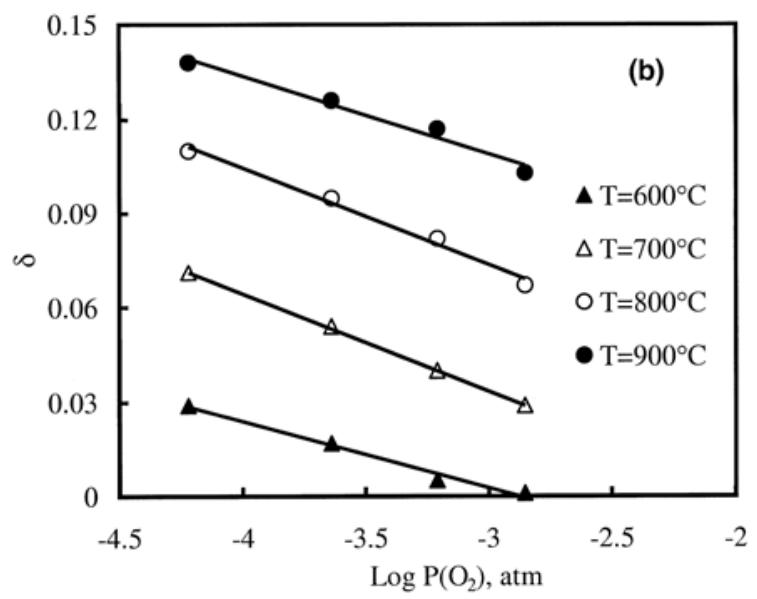

Figure 6.4 Oxygen non-stoichiometry as a function of oxygen partial pressure for $\mathrm{La}_{0.6} \mathrm{Sr}_{0.4} \mathrm{Co}_{0.2} \mathrm{Fe}_{0.8} \mathrm{O}_{3-\delta}{ }^{74}$

LSCF conductivity variation with oxygen partial pressure has also been observed in our study and the results were shown in Figure 6.5. The results revealed that the conductivity value of LSCF decreases linearly with $\log P_{\mathrm{O}_{2}}$. We could further deduce that LSCF is a p-type conductor (region III in Figure 6.3) within the oxygen partial pressure range in our study.

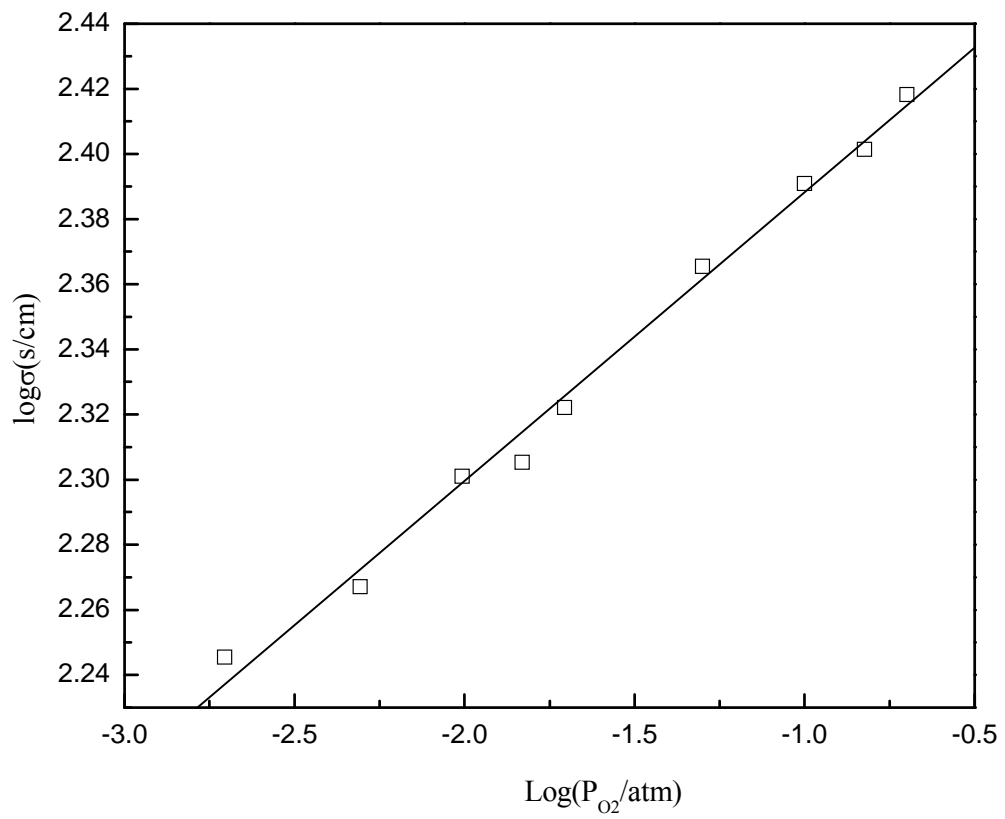

Figure 6.5 Conductivity of $\mathrm{La}_{0.6} \mathrm{Sr}_{0.4} \mathrm{Co}_{0.2} \mathrm{Fe}_{0.8} \mathrm{O}_{3 \pm \delta}$ at $800^{\circ} \mathrm{C}$ as a function of oxygen partial pressure 


\subsection{Electrical conductivity relaxation testing results}

To examine relaxation behavior through a range of absolute oxygen partial pressures, ECR measurements were performed at different oxygen partial pressure levels including $0.20,0.15$, 0.10, 0.05 and $0.02 \mathrm{~atm}$. The step change $\left(\left|\Delta \log P_{O_{2}}\right|\right)$ is smaller than 0.1 for all these conditions. And for each level, we observed both of the oxidation and reduction process for LSCF. In other words, the testing atmosphere was switched forward and backward between high and low oxygen partial pressure.

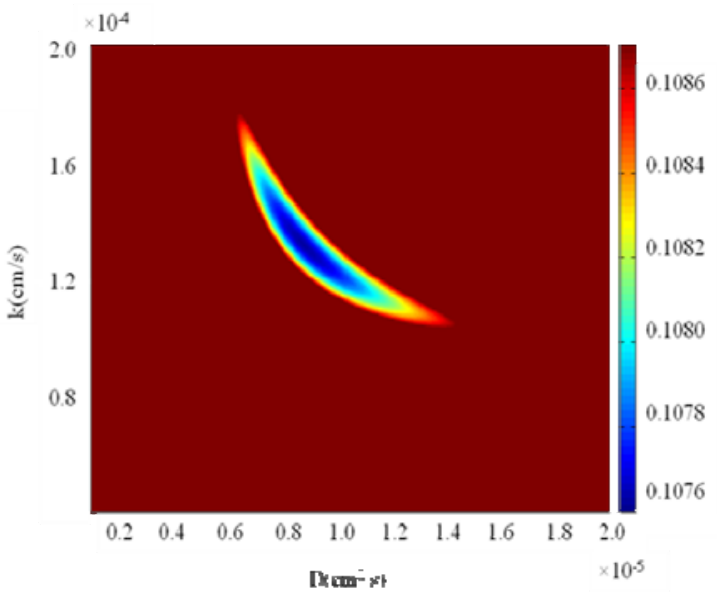

(a) $0.20 \mathrm{~atm} \rightarrow 0.18 \mathrm{~atm}$

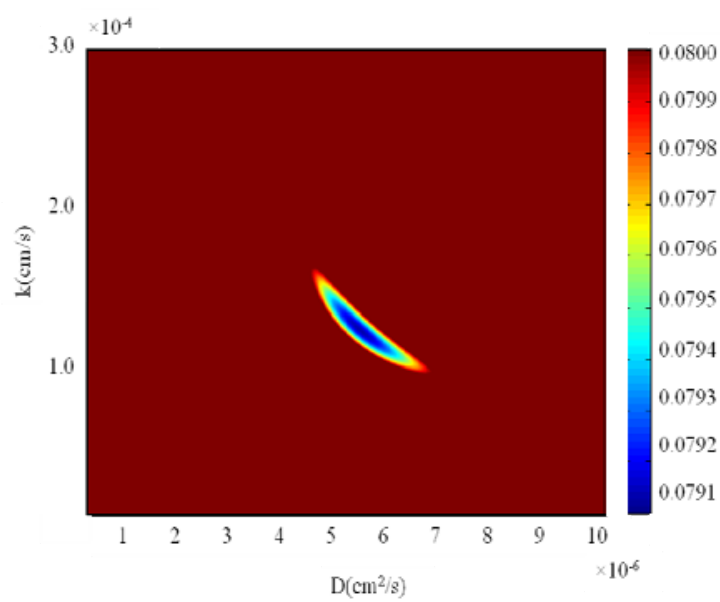

(c) $0.15 \mathrm{~atm} \rightarrow 0.14 \mathrm{~atm}$

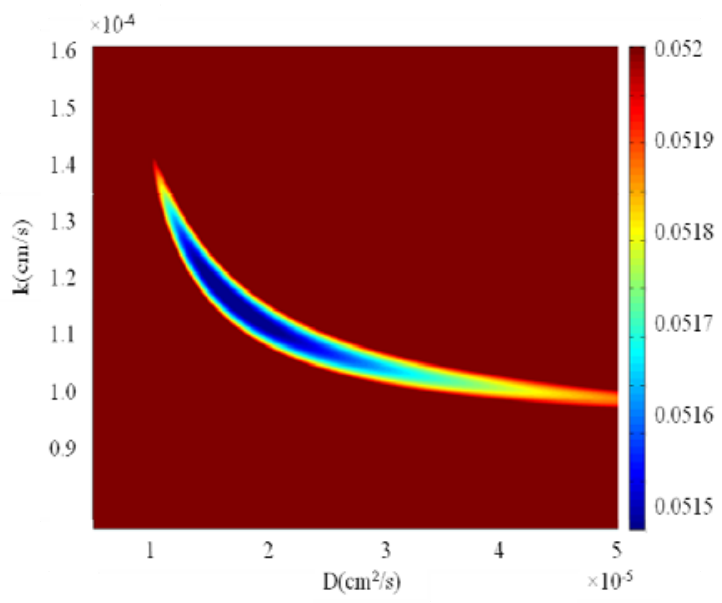

(b) $0.18 \mathrm{~atm} \rightarrow 0.20 \mathrm{~atm}$

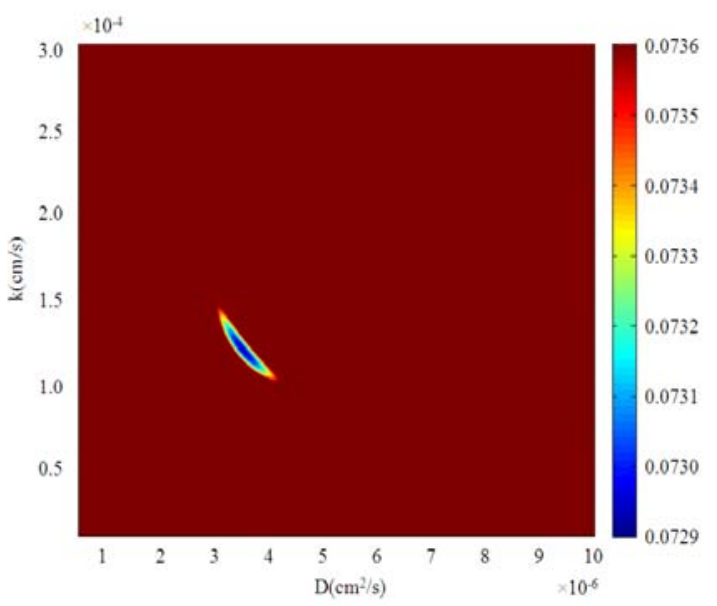

(d) $0.14 \mathrm{~atm} \rightarrow 0.15 \mathrm{~atm}$ 


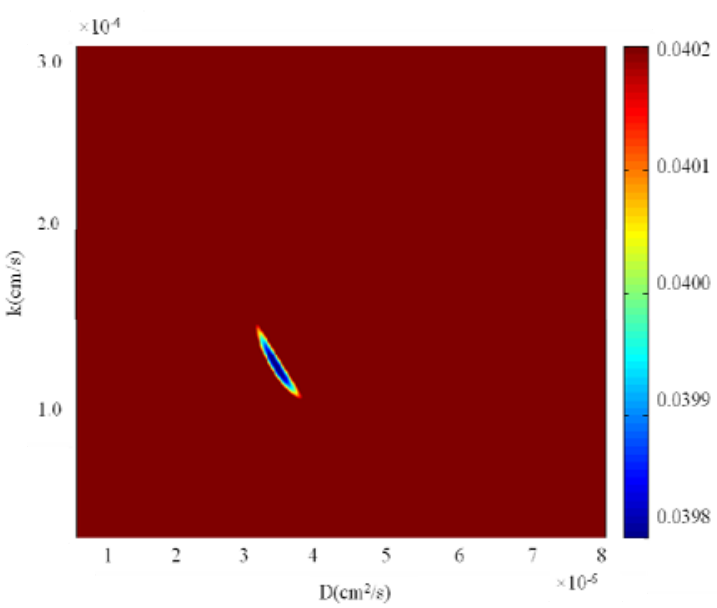

(e) $0.10 \mathrm{~atm} \rightarrow 0.09 \mathrm{~atm}$

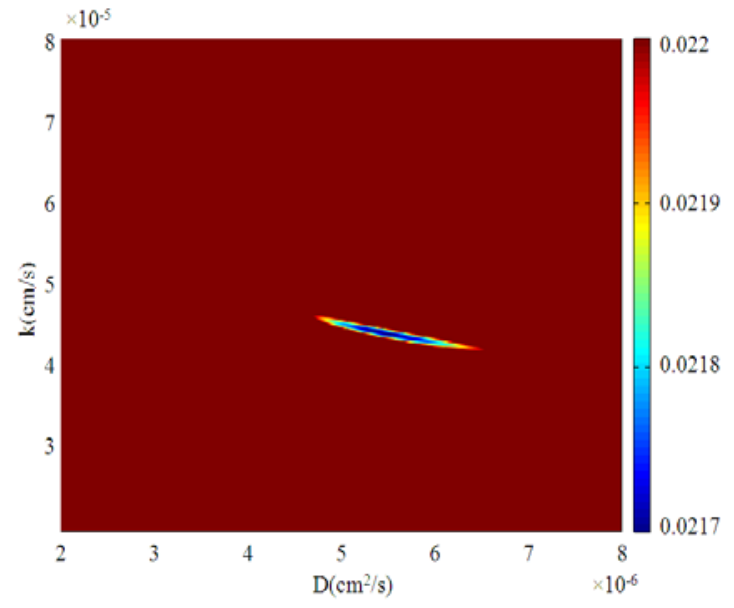

(g) $0.05 \mathrm{~atm} \rightarrow 0.04 \mathrm{~atm}$

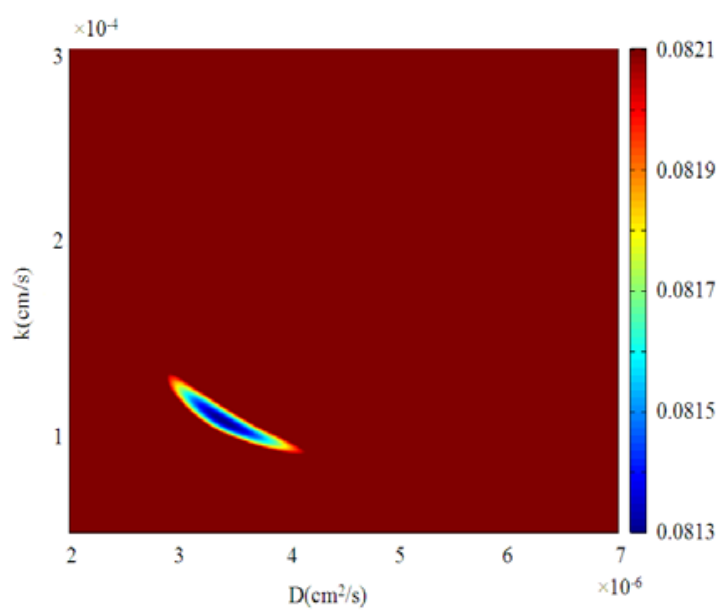

(f) $0.09 \mathrm{~atm} \rightarrow 0.10 \mathrm{~atm}$

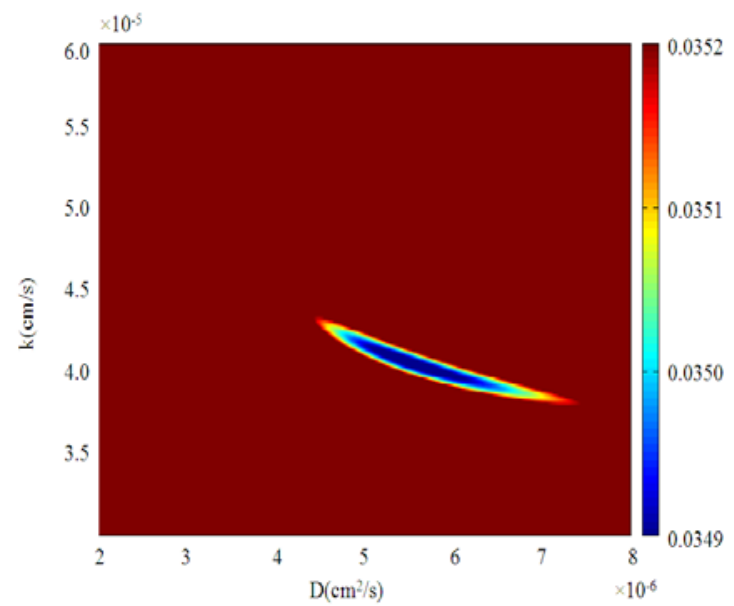

(h) $0.04 \mathrm{~atm} \rightarrow 0.05 \mathrm{~atm}$ 


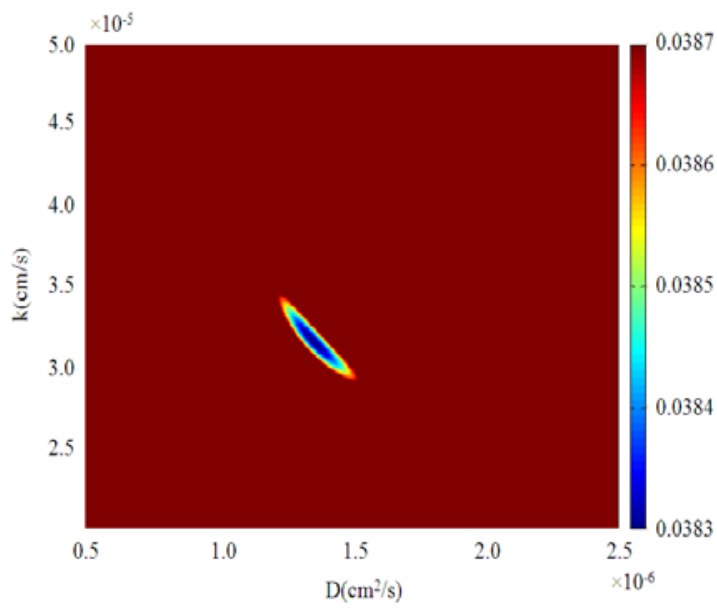

(i) $0.020 \mathrm{~atm} \rightarrow 0.018 \mathrm{~atm}$

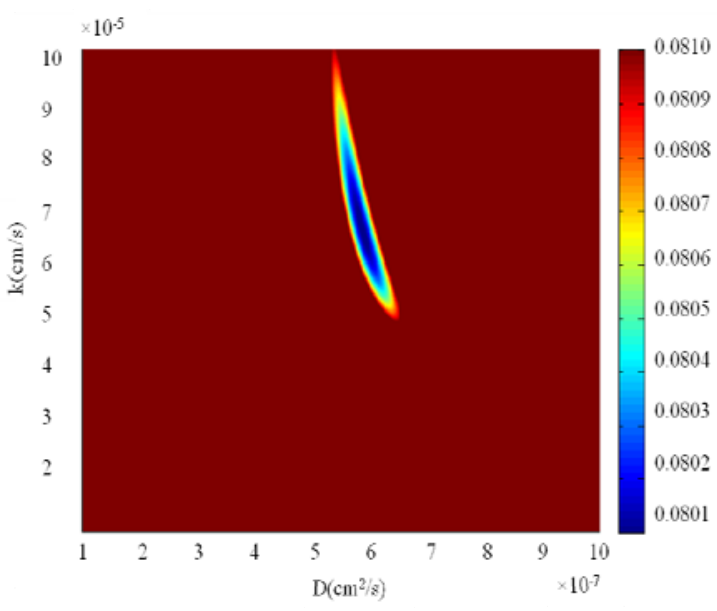

(j) $0.018 \mathrm{~atm} \rightarrow 0.020 \mathrm{~atm}$

Figure 6.6 Fitting error color maps for LSCF under different oxygen partial pressure

Figure 6.6 presents the results for simultaneously fitting two relaxation profiles at the chosen (D, k) plane. In Figure $6.6, f$ values are revealed by the color bars within $101 \%$ of its minimum value. According to the fitting results, both of the oxygen surface exchange and bulk diffusion coefficients will decrease with the oxygen partial pressure decreasing.

\subsection{Discussion}

\subsubsection{Oxygen distribution during relaxation process}

Oxygen enters or exits the perovskite lattice to achieve the saturated concentration during the relaxation process. The oxygen concentration equilibrium in a new environment is obtained through surface exchange and bulk diffusion. The relaxation process can be divided into three different types according to the main rate limiting step, namely bulk diffusion control, surface exchange control and mixed control. Oxygen distributions for these three relaxation types are indicated in Figure 6.7. For bulk diffusion control, the surface exchange process can be considered to reach the new equilibrium immediately after the oxygen partial pressure change. 
Bulk oxygen gets saturated gradually due to the concentration difference. On the contrary, for surface exchange control type, the diffusion process can be ignored. Oxygen accumulates or depletes from the material gradually by surface exchange. At last, for mixed control relaxation, both of the surface and bulk oxygen concentration will achieve new equilibrium gradually. By calculation of the characteristic length $l_{c}$ with the fitted $D$ and $k$ values, the relaxation processes observed for LSCF all belong to the mixed control type. In the following part, we will discuss the surface and bulk transport process individually.
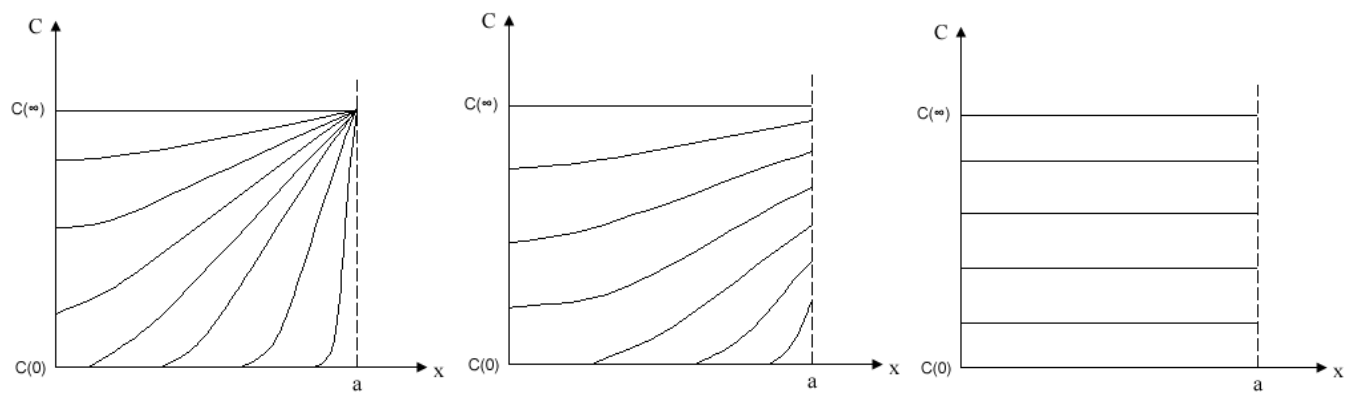

(a) Bulk diffusion control

(b). Mixed control

(c). Surface exchange control

Figure 6.7 Oxygen distributions with different rate-limited steps

\subsubsection{Oxygen partial pressure effects on relaxation process}

\subsubsection{Oxygen surface exchange $(k)$}

The oxygen surface exchange process can be further divided into at least two sub-steps, oxygen adsorption and reduction. Therefore, both the oxygen partial pressure and activated surface adsorption site concentration can affect oxygen surface exchange rate. Generally, in an isothermal system, we can consider LSCF's adsorption site concentration as a constant. We focus analysis on oxygen partial pressure effects on the surface exchange process.

The overall oxygen surface exchange reaction is given by: 


$$
\frac{1}{2} O_{2}+2 e^{-}+V_{o}^{\cdot *} \underset{k_{2}}{\stackrel{k_{1}}{\rightleftarrows}} O_{o}^{x}
$$

$k_{1}$ and $k_{2}$ are forward and reverse reaction rate constants. If $\left[V_{o}^{*}\right]$ and $C(\infty)$ separately stand for oxygen vacancy and lattice oxygen ion concentration when the new equilibrium has been achieved, the forward reaction rate should be equal to the reverse rate:

$$
k_{1} P_{O_{2}}^{1 / 2}\left[V_{o}^{\cdot \cdot}\right]=k_{2} C(\infty)
$$

where $P_{O_{2}}$ is the final state oxygen partial pressure. Additionally, for non-equilibrium conditions, the net surface exchange rate should equal to the difference between the forward and reverse reaction rate, given by:

$$
k_{1} P_{O_{2}}^{1 / 2}\left[V_{o}^{\cdot \cdot}\right]^{\prime}-k_{2} C_{s}=k\left[C(\infty)-C_{s}\right]
$$

$\left[V_{o}^{\cdot *}\right]$ and $C_{s}$ represent oxygen vacancy and oxygen ion concentration at non-equilibrium conditions. From Equation 6.17, it seems that surface exchange coefficient $k$ depends on the final $P_{\mathrm{O}_{2}}$, oxygen vacancy and oxygen ion concentration. However, due to electro-neutrality and site conservation, equation 6.18 can be derived:

$$
\left[V_{o}^{\cdot \cdot}\right]^{\prime}=\left[V_{o}^{\cdot \cdot}\right]+C(\infty)-C_{s}
$$

The mathematical expression of $\mathrm{k}$ can be transformed as given below in Equation 6.18 utilizing the relationship shown in Equation 6.19: 


$$
\begin{aligned}
& k=\frac{k_{1} P_{O_{2}}^{1 / 2}\left[V_{o}^{*}\right]^{\prime}-k_{2} C_{s}}{\left[C(\infty)-C_{s}\right]}=k_{1} P_{O_{2}}^{1 / 2}+\frac{k_{1} P_{O_{2}}^{1 / 2}\left[V_{o}^{*}\right]-k_{2} C_{s}}{\left[C(\infty)-C_{s}\right]} \\
& =k_{1} P_{O_{2}}^{1 / 2}+\frac{k_{2} C(\infty)-k_{2} C_{s}}{\left[C(\infty)-C_{s}\right]}=k_{1} P_{O_{2}}^{1 / 2}+k_{2}
\end{aligned}
$$

Because $k_{1}$ and $k_{2}$ are constants at a given temperature, it can be deduced that the oxygen surface exchange coefficient only depends on final $P_{\mathrm{O}_{2}}$. Furthermore, it indicates that $k$ is a function of $P_{O_{2}}^{1 / 2}$.

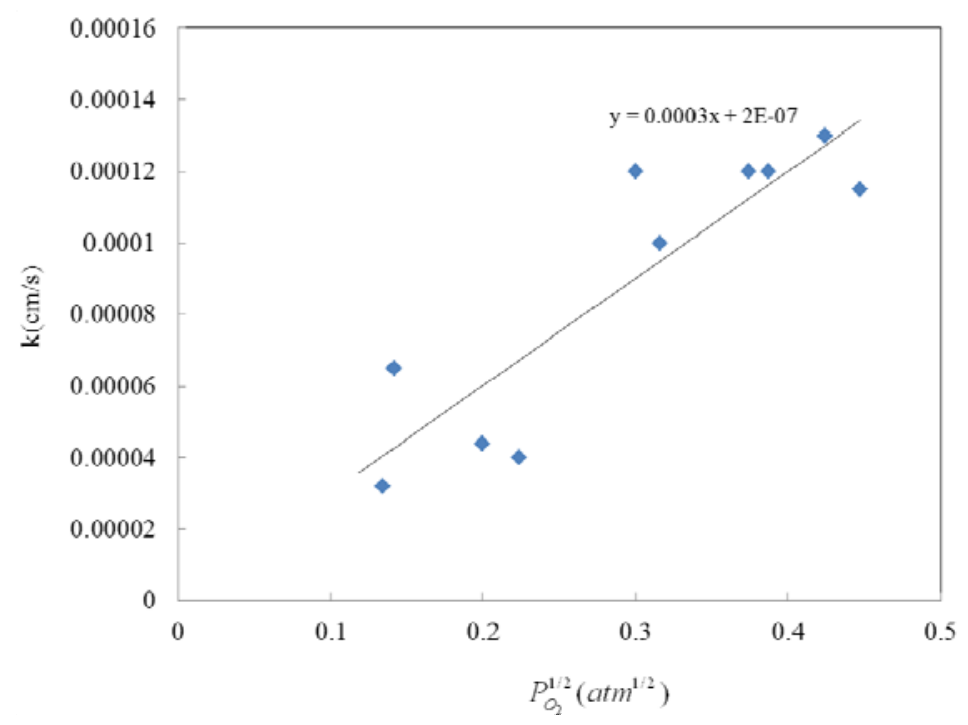

Figure 6.8 Oxygen surface exchange coefficient for $\mathrm{La}_{0.6} \mathrm{Sr}_{0.4} \mathrm{Co}_{0.2} \mathrm{Fe}_{0.8} \mathrm{O}_{3-\delta}$ at $800^{\circ} \mathrm{C}$ as a function of oxygen partial pressure obtained from conductivity relaxation

The fitted surface exchange coefficients according to $P_{O_{2}}^{1 / 2}$ were plotted in Figure 6.8. Using these fitted $k$ values, oxygen reduction reaction constants of $\mathrm{La}_{0.6} \mathrm{Sr}_{0.4} \mathrm{Co}_{0.2} \mathrm{Fe}_{0.8} \mathrm{O}_{3-\delta}$ at $800^{\circ} \mathrm{C}$ can be obtained by fitting Equation 6.19, as the solid line shown in Figure 6.8. The results revealed 
$k_{l}=0.0003 \mathrm{~cm} / \mathrm{atm}^{1 / 2} \cdot \mathrm{s}$ and $k_{2}=2 \times 10^{-7} \mathrm{~cm} / \mathrm{s}$. The most important application for the reaction rate constants is to estimate the net oxygen ionic flow rate and further to estimate exchange current for SOFCs cathode. Besides, the activation energy can be calculated using the deduced reaction rate constants under different temperature. By comparing the activation energies for the forward and backward reactions, we can conclude which reaction is easier to occur.

6.5.2.2 Bulk diffusion coefficient $(D)$

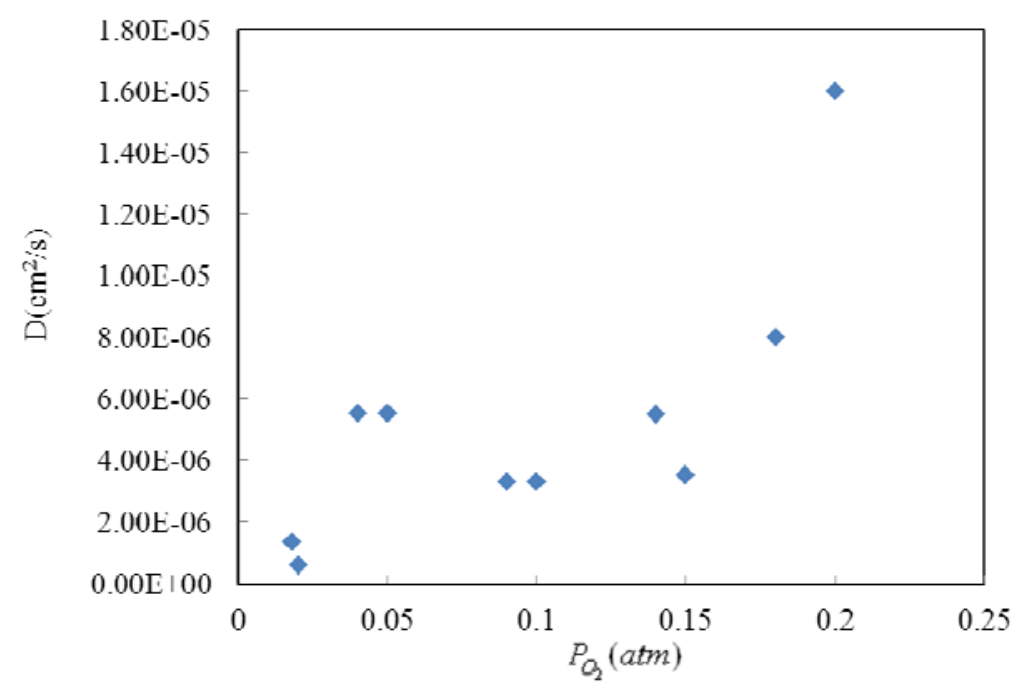

Figure 6.9 Oxygen bulk diffusion coefficient for $\mathrm{La}_{0.6} \mathrm{Sr}_{0.4} \mathrm{Co}_{0.2} \mathrm{Fe}_{0.8} \mathrm{O}_{3-\delta}$ at $800^{\circ} \mathrm{C}$ as a function of oxygen partial pressure obtained from conductivity relaxation

The oxygen bulk diffusion coefficient $(D)$ for $\mathrm{La}_{0.6} \mathrm{Sr}_{0.4} \mathrm{Co}_{0.2} \mathrm{Fe}_{0.8} \mathrm{O}_{3-\delta}$ as a function of oxygen partial pressure is shown in Figure 6.9. The decreasing slope of observed bulk diffusion coefficient is smaller than the surface exchange coefficient. When oxygen partial pressure is below $0.15 \mathrm{~atm}$, the bulk diffusion coefficient varies in a narrow band and it decreases again until $P_{\mathrm{O}_{2}}$ is lower than $0.02 \mathrm{~atm}$. 
Based on random walk theory, J. A. Kilner ${ }^{75}$ deduced the relation between oxygen self diffusion coefficient and bulk vacancy concentration as below:

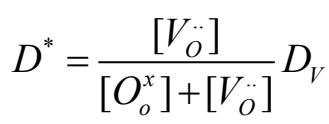

Mizusaki ${ }^{76}$ had pointed out that oxygen vacancy diffusivity $\left(D_{V}\right)$ varies little in the perovskite oxides. On the other hand, the oxygen vacancy concentration can be given as an analytical function of $\delta$. Mantzavinos ${ }^{74}$ and Dalslet ${ }^{77}$ studied the non-stoichiometry value variation under different oxygen partial pressures. Their experimental results revealed that $\delta$ was linearly dependent on the value of $P_{\mathrm{O}_{2}}$ with a slope of -0.016 , approximately. Therefore, if oxygen vacancies are randomly distributed, oxygen bulk diffusion coefficient should slightly increase with $P_{\mathrm{O}_{2}}$ decreasing. A possible explanation for the results observed in our study is the oxygen vacancy ordering which depends both on oxygen partial pressure and temperature ${ }^{78,79}$.

\subsection{Concluding remarks}

In this chapter, oxygen transportation behavior in $\mathrm{La}_{0.6} \mathrm{Sr}_{0.4} \mathrm{Co}_{0.2} \mathrm{Fe}_{0.8} \mathrm{O}_{3-\delta}$ has been investigated. First, we analyzed its defects chemistry and conductivity type under different oxygen partial pressure. The LSCF was theoretically considered as a p-type semiconductor under our experimental conditions and its conductivity decreases with decreasing $P_{\mathrm{O}_{2}}$.

Besides, oxygen surface exchange and bulk diffusion coefficients of $\mathrm{La}_{0.6} \mathrm{Sr}_{0.4} \mathrm{Co}_{0.2} \mathrm{Fe}_{0.8} \mathrm{O}_{3-\delta}$ were obtained by applying the improved ECR data analyzing method. The relaxation data profile obtained from ECR testing revealed hysteresis with the reduction process requiring more equilibration time than the oxidation process for the same oxygen partial pressure changing step. 
And both of the oxygen surface exchange and bulk diffusion coefficients will decrease with the decrease in oxygen partial pressure.

Further, the relaxation process has been discussed. And the deduced conclusion implies that the oxygen surface exchange coefficient only depends on the final oxygen partial pressure following the $P_{O_{2}}^{1 / 2}$ law. Oxygen reduction reaction constants can be generated then and the results reveal that oxygen entering the LSCF lattice is a lower energy process than leaving at $800^{\circ} \mathrm{C}$. On the other hand, oxygen bulk diffusion coefficient was considered to be influenced by the oxygen vacancy concentration and the ordering degree. The decreasing slope of the observed bulk diffusion coefficient is smaller than the surface exchange coefficient. 


\section{Oxygen transport behavior investigation for infiltrated SOFC cathode}

\subsection{Introduction}

To improve the SOFC cathode performance, infiltration processes have been used to fabricate composite cathodes by introducing a thin film of catalyst onto a porous supporting structure. The infiltrated cathodes provide superior performance compared to single phase cathodes due to electrochemically active area extension. Infiltrated material and amount, sintering process and temperature are considered as the factors governing infiltrated cathode performance. However, due to lack of understanding for the infiltrated cathode mechanism, it's difficult to distinguish the independent effects of each parameter and to optimize the manufacturing process effectively.

In this chapter, we will develop the ECR technique to characterize oxygen transport behavior at the interface of infiltrate/cathode material. Two commonly used infiltrated materials $\mathrm{Ce}_{0.8} \mathrm{Sm}_{0.2} \mathrm{O}_{1.9}$ and $\mathrm{La}_{0.6} \mathrm{Sr}_{0.4} \mathrm{CoO}_{3-\delta}$ were investigated. $\mathrm{La}_{0.6} \mathrm{Sr}_{0.4} \mathrm{Co}_{0.2} \mathrm{Fe}_{0.8} \mathrm{O}_{3-\delta}$ was chosen as the cathode backbone for its wide application in commercial systems. Based on our previous study for LSCF, oxygen exchange coefficients at SDC/LSCF or LSC/LSCF interface are reported in this chapter. Results are used to compare performance improvement of the two infiltrated cathodes.

\subsection{Sample preparation and characterization}

\subsubsection{Sample preparation}

A spin coating method was utilized to prepare the multilayer sample for infiltrated cathode investigation. The spin coating solution contains low concentrations of organic binders as $(0.002 \mathrm{~g} / \mathrm{mL})$, PEI $(6.7 \times 10-4 \mathrm{~g} / \mathrm{mL})$ and ethyl cellulose $(6.7 \times 10-4 \mathrm{~g} / \mathrm{mL})$. The solvent is ethanol. 
The speed for spin coating was set as $1200 \mathrm{rpm}$ for $10 \mathrm{~s}$ following $2500 \mathrm{rpm}$ for $40 \mathrm{~s}$. Detailed sample preparation processes are illustrated as below.

First, $\mathrm{La}_{0.6} \mathrm{Sr}_{0.4} \mathrm{Co}_{0.2} \mathrm{Fe}_{0.8} \mathrm{O}_{3-\delta}$ powder ((NexTech Materials, Ltd.) was pressed axially at $250 \mathrm{MPa}$ using a $19 \mathrm{~mm}$ (diameter) die. For SDC/LSCF multilayer sample, the LSCF pellet was sintered at $1200^{\circ} \mathrm{C}$ for $2 \mathrm{~h}$. Then samples are spin coated five times on each side and sintered at $1350^{\circ} \mathrm{C}$ for 2h. For the LSC/LSCF multilayer sample, considering LSC is unstable, LSCF pellet was first sintered at $1350^{\circ} \mathrm{C}$ for $2 \mathrm{~h}$ to make a dense substrate. A spin coated LSC layer was applied five times on each side of the pellet and the multi-layers were co-sintered at a lower temperature. We tried two temperatures $1200^{\circ} \mathrm{C}$ and $1100^{\circ} \mathrm{C}$ for co-sintering. However, for the samples processed at $1200{ }^{\circ} \mathrm{C}$ iron diffused from substrate to surface, as shown in Figure 7.1. Therefore, the LSC/LSCF multilayer sample was co-sintered at $1100^{\circ} \mathrm{C}$. 


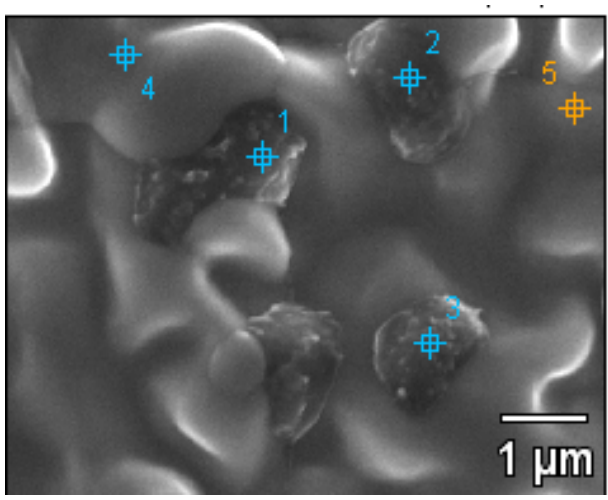

\begin{tabular}{|c|c|c|c|c|c|c|}
\hline Element (at.\%) & $C$ & $O$ & $\mathrm{Fe}$ & $\mathrm{Co}$ & $S r$ & $L a$ \\
\hline 1 & 1.28 & 56.79 & 4.68 & 35.55 & 0.78 & 0.93 \\
\hline 2 & 1.51 & 60.53 & 4.09 & 32.61 & 0.53 & 0.73 \\
\hline 3 & 1.60 & 65.13 & 3.28 & 28.28 & 0.90 & 0.81 \\
\hline 4 & 3.48 & 63.81 & 9.86 & 7.10 & 7.94 & 7.81 \\
\hline 5 & 2.09 & 39.84 & 22.08 & 11.72 & 8.81 & 15.46 \\
\hline
\end{tabular}

Figure 7.1 Surface morphologies and surface chemical composition of LSC/LSCF pellet

7.2.2 Surface and cross section morphologies 


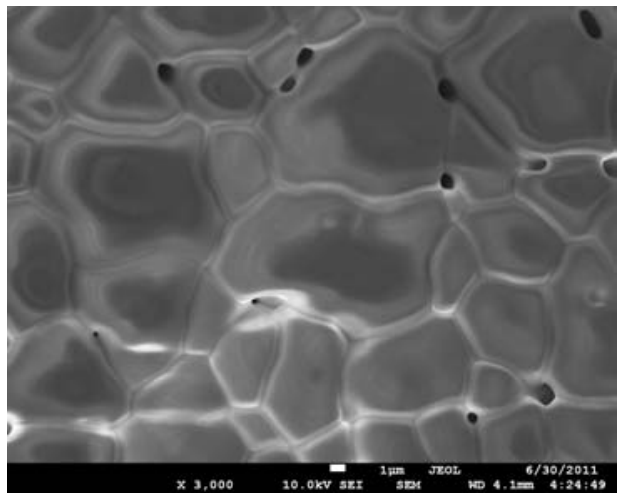

(a) Surface morphology of spin coated SDC
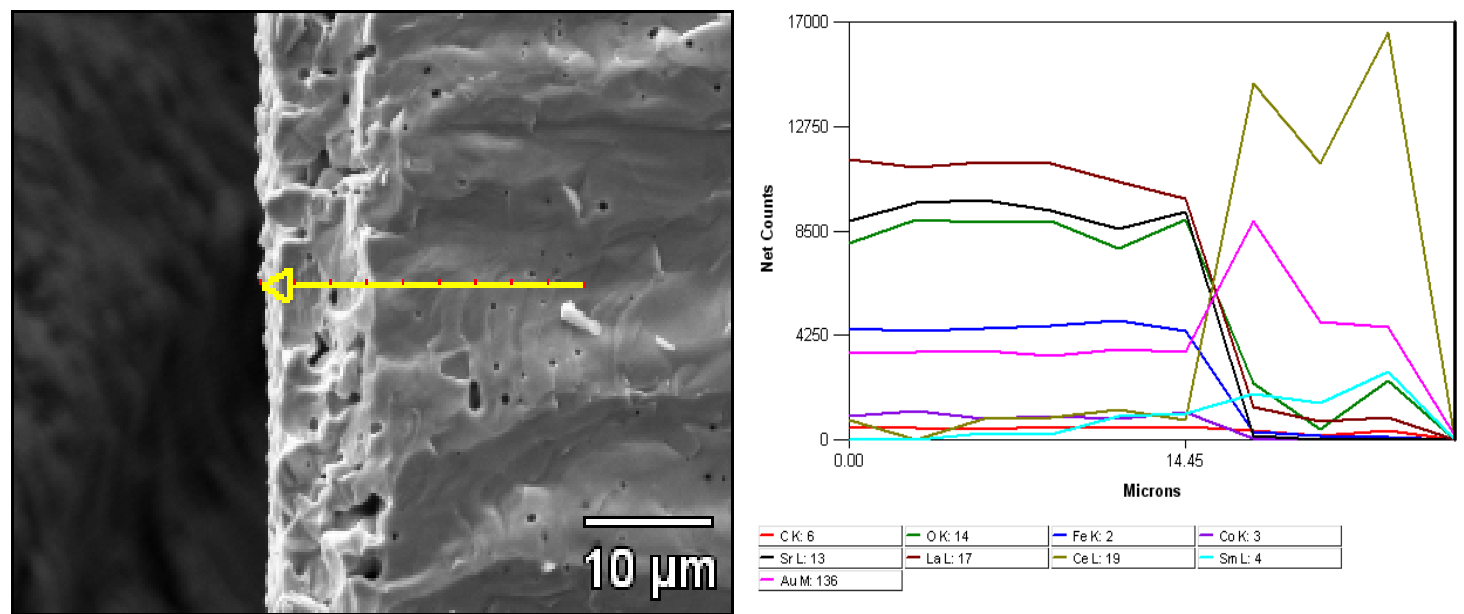

(b) Cross section and line scanning results

Figure 7.2 Surface and cross section morphologies of spin coated SDC sample

Figure 7.2 presents the surface and cross section morphologies of the SDC/LSCF multilayer sample. From Figure 7.2(a), SDC spin coated layer is dense and its average grain size is about $10 \mu \mathrm{m}$. And the SDC film thickness is approximately $6 \mu \mathrm{m}$. 


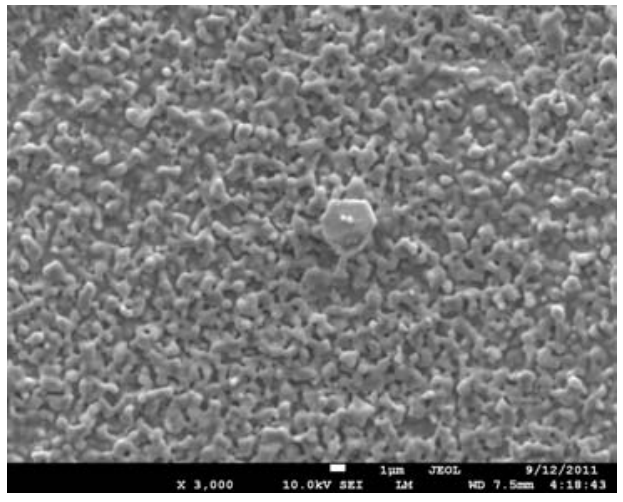

(a) Surface morphology of spin coated LSC
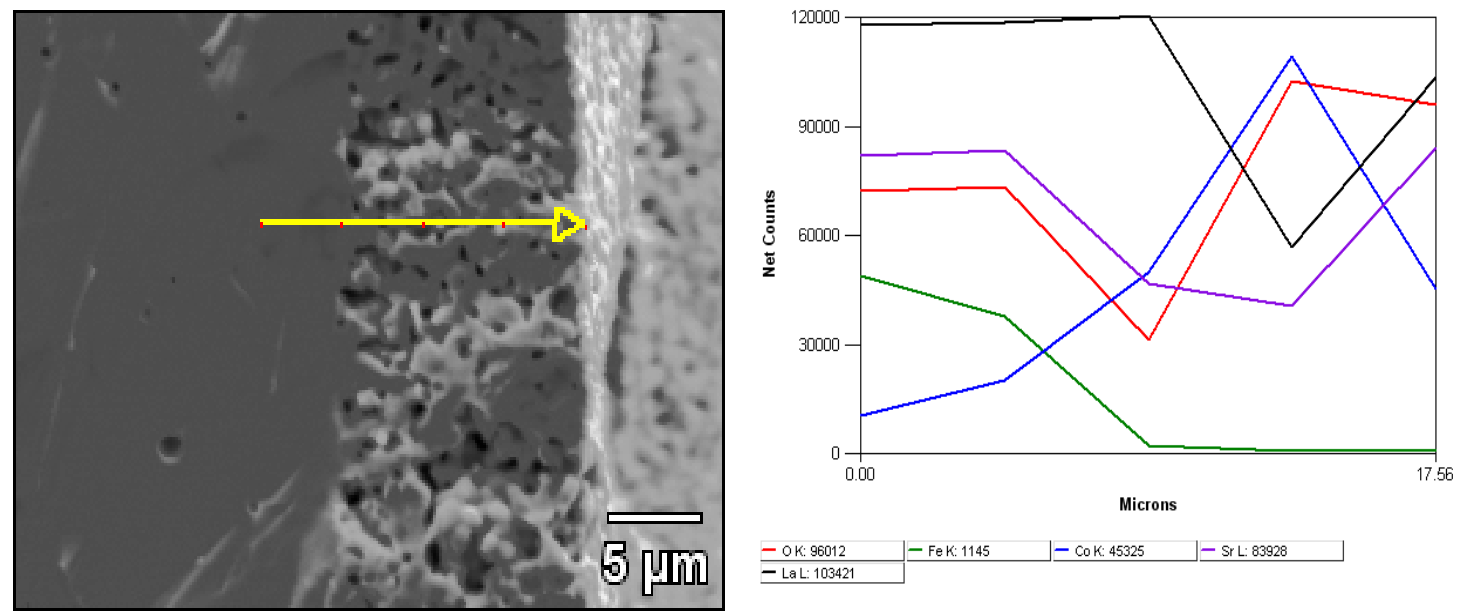

(b) Cross section and line scanning results

Figure 7.3 Surface and cross section morphologies of spin coated LSC sample

Images for LSC/LSCF multilayer sample are presented in Figure 7.3. No iron diffusion was detected by applying $1100^{\circ} \mathrm{C}$ as the co-sinter temperature. However, formed LSC thin film is porous and it needs to be correlated to LSC's intrinsic oxygen kinetic parameters. 


\subsection{Oxygen transport behavior in infiltrated materials}

\subsection{1 $\mathrm{Ce}_{0.8} \mathrm{Sm}_{0.2} \mathrm{O}_{1.9}(\mathrm{SDC})$}

Samaria-doped ceria (SDC) is considered as a promising electrolyte candidate for intermediatetemperature SOFCs because of its excellent oxygen ion conductivity. It has also been used as an infiltration material to improve cathode performance ${ }^{80,81}$. Oxygen vacancy formation can be introduced either by intrinsic defects or extrinsic reaction via samaria doping.

$$
\begin{gathered}
\mathrm{O}_{O}^{x} \rightarrow V_{\ddot{O}}+2 e^{\prime}+\frac{1}{2} \mathrm{O}_{2} \\
\mathrm{Sm}_{2} \mathrm{O}_{3} \stackrel{\mathrm{CeO}_{2}}{\longrightarrow} 2 \mathrm{Sm}_{\mathrm{Ce}}^{\prime}+V_{O}^{\ddot{O}}+3 \mathrm{O}_{O}^{x}
\end{gathered}
$$

Matsui $^{82}$ et al. investigated electronic and ionic conductivity variation with oxygen partial pressure for $\mathrm{Ce}_{0.8} \mathrm{Sm}_{0.2} \mathrm{O}_{1.9}$, as shown in Figure 7.4. The results revealed that at $800^{\circ} \mathrm{C}$ ionic conductivity will dominate and approximately keep constant in the range of oxygen partial pressure from $1 \mathrm{~atm}$ to $10^{-12} \mathrm{~atm}$. Therefore, no conductivity relaxation can be detected under our testing environment. Considering its high oxygen ion conductivity and the thickness of the SDC spin coated film in our study, oxygen diffusion process in SDC was ignored. 


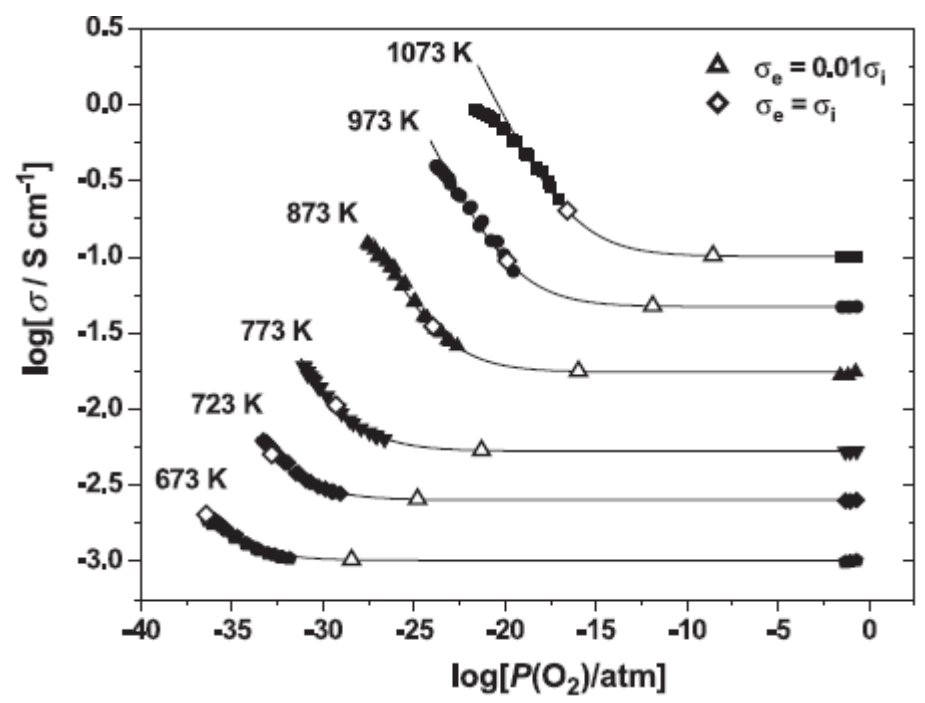

Figure 7.4 Total conductivity dependence on oxygen partial pressure of SDC

\subsection{2 $\mathrm{La}_{0.6} \mathrm{Sr}_{0.4} \mathrm{CoO}_{3-\delta}(\mathrm{LSC})$}

Strontium substituted lanthanum cobaltite is a perovskite-type oxide with higher electronic and oxide ion conductivity than LSCF. However, its high thermal expansion property and chemical instability when compared to the electrolyte material YSZ inhibit the direct use of LSC as SOFCs cathode. Application of LSC by the technique of infiltration can utilize the LSC's advantages and at the same time avoid those problems.

Commercial $\mathrm{La}_{0.6} \mathrm{Sr}_{0.4} \mathrm{CoO}_{3-\delta}$ (AGC Seimi Chemical Co., Ltd. Chigasaki-city, Kanagawa, Japan) was investigated. As when preparing LSCF pellets, the powders were axially pressed at $250 \mathrm{MPa}$ using a $19 \mathrm{~mm}$ (diameter) die. The pellets then were sintered at $1200^{\circ} \mathrm{C}$ in air for $2 \mathrm{~h}$ following progressively polished on both sides using silicon carbide polishing papers followed by polycrystalline diamond suspension up to $1 \mu \mathrm{m}$ (PACE Technologies, Tucson, Arizona, USA). And the samples were verified over $95 \%$ of theoretical density. 


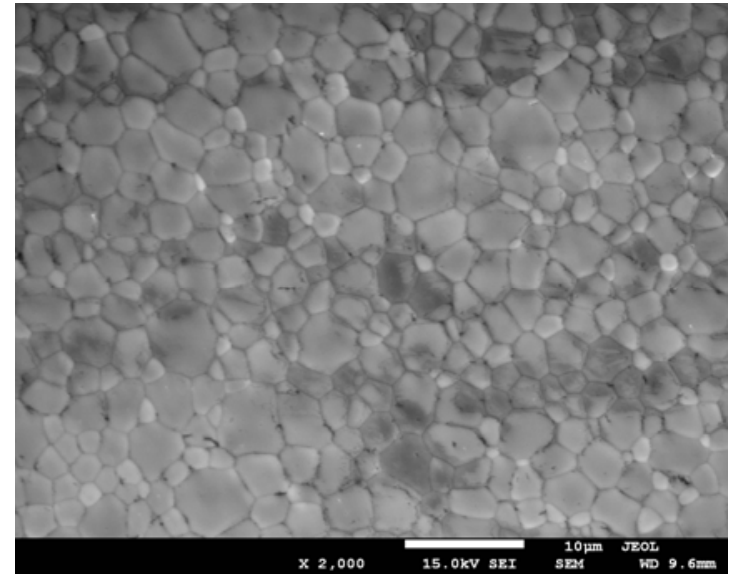

(a)

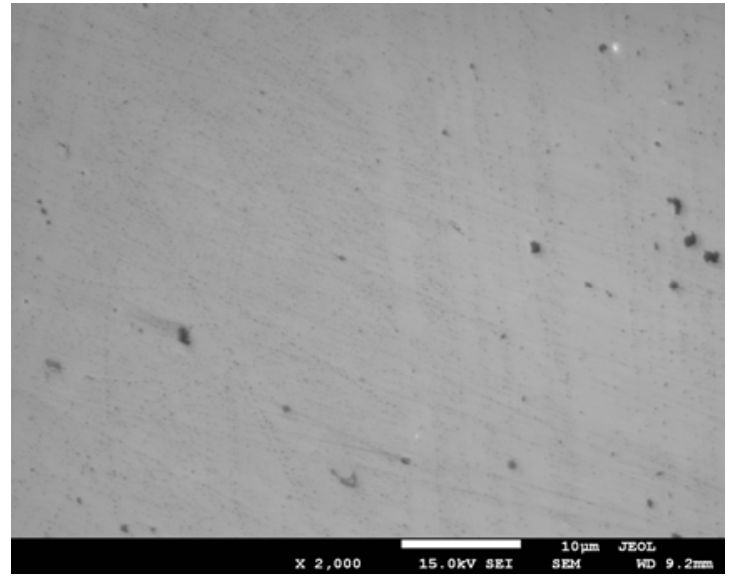

(b)

\begin{tabular}{lllll}
\hline & La & Sr & Co & O \\
& & & & \\
\hline at. $\%$ & 9.70 & 8.93 & 21.1 & 60.27
\end{tabular}

Figure 7.5 Surface morphologies and surface chemical composition of sintered LSC pellet

(a) Before polishing (b) After polishing

Figure 7.5 showed the surface image and chemical composition of the LSC sample used in this study. The average grain size is approximate to $3 \mu \mathrm{m}$. Atomic ratio of lanthanum and strontium decreased a little compared to 6:4.

Similar to the investigation of LSCF in Chapter 6 , the electrical conductivity relaxation technique was utilized to characterize the oxygen transport behavior of LSC. However, it has been found that the conductivity relaxation response of LSC is quicker than LSCF. The total relaxation time was around 100s when applying same oxygen partial pressure step change. To shorten the flash time ratio, bigger oxygen partial step change was used for testing LSC material. 


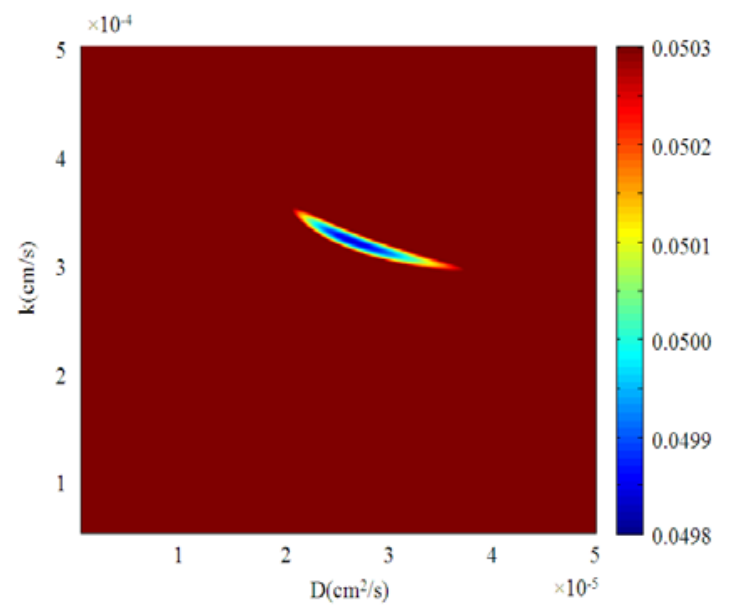

(a) $0.20 \mathrm{~atm} \rightarrow 0.15 \mathrm{~atm}$

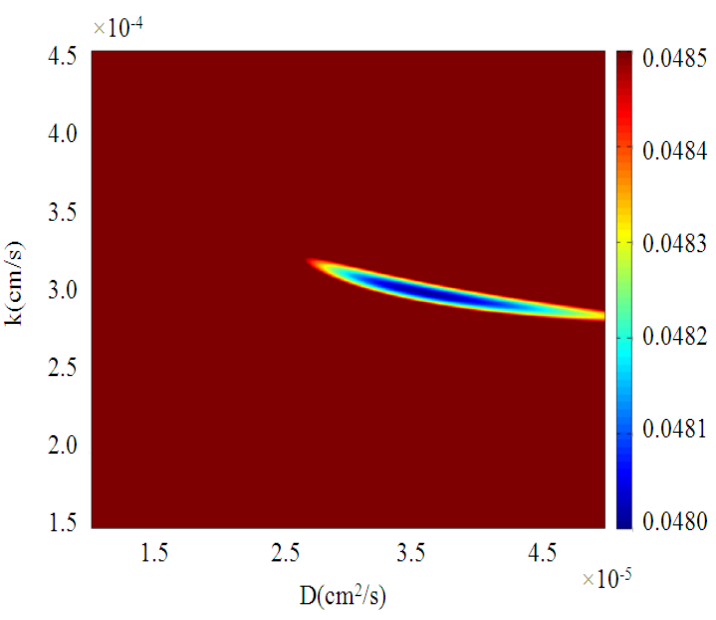

(c) $0.15 \mathrm{~atm} \rightarrow 0.10 \mathrm{~atm}$

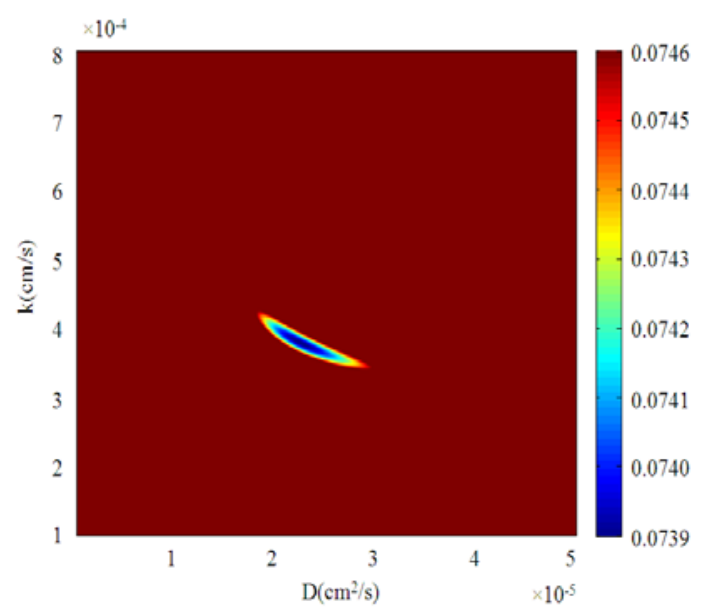

(b) $0.15 \mathrm{~atm} \rightarrow 0.20 \mathrm{~atm}$

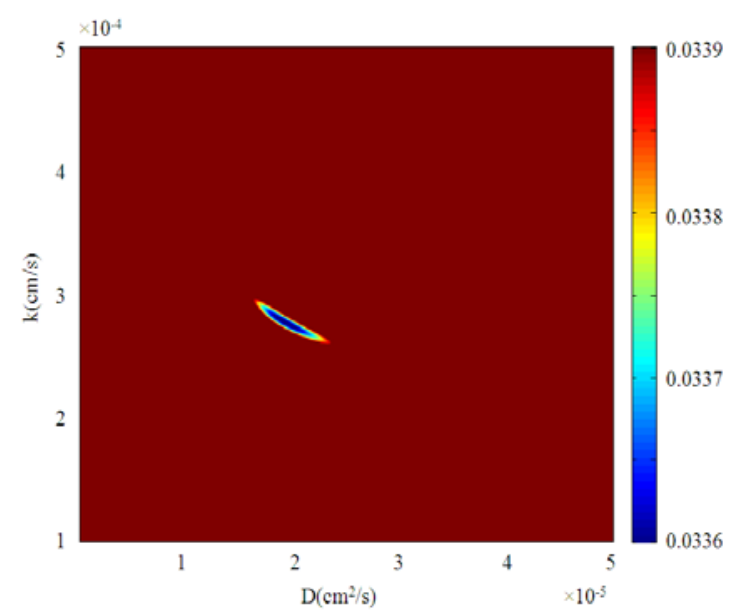

(d) $0.10 \mathrm{~atm} \rightarrow 0.15 \mathrm{~atm}$ 


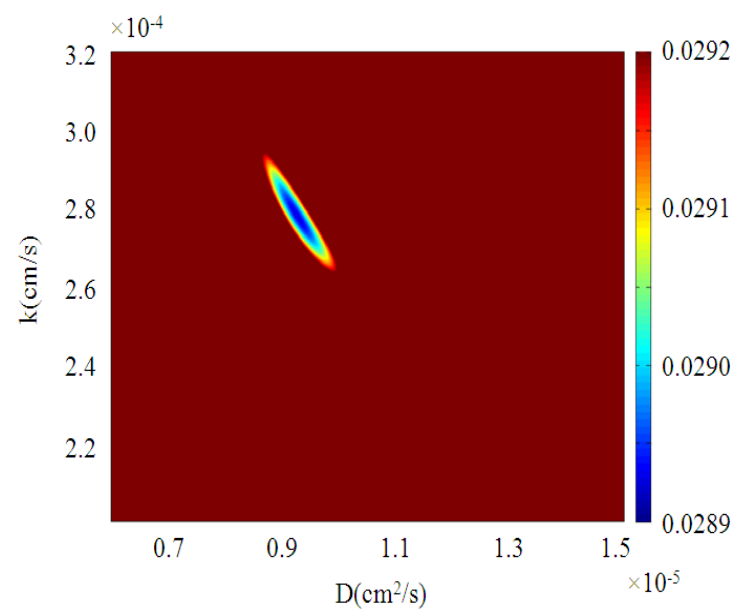

(e) $0.10 \mathrm{~atm} \rightarrow 0.05 \mathrm{~atm}$

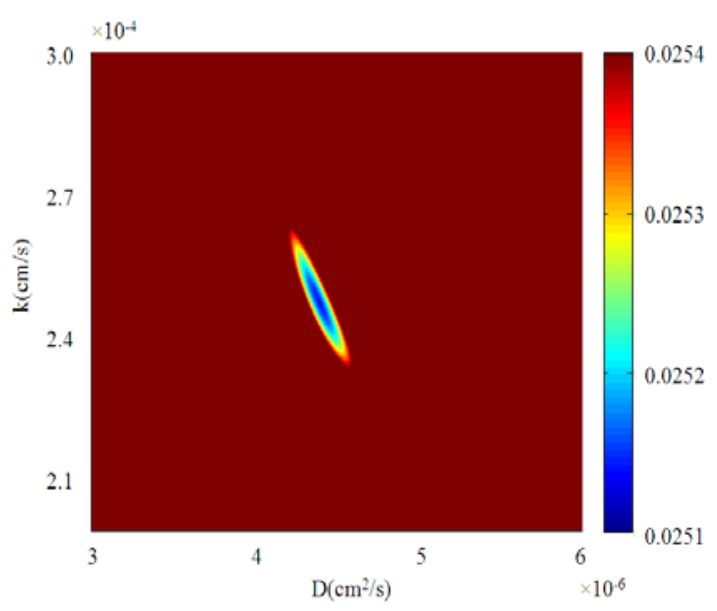

(g) $0.05 \mathrm{~atm} \rightarrow 0.02 \mathrm{~atm}$

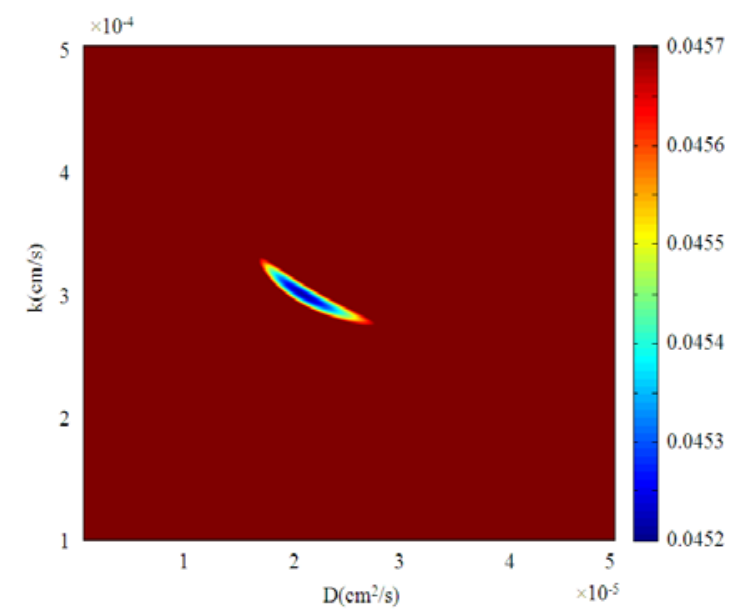

(f) $0.05 \mathrm{~atm} \rightarrow 0.10 \mathrm{~atm}$

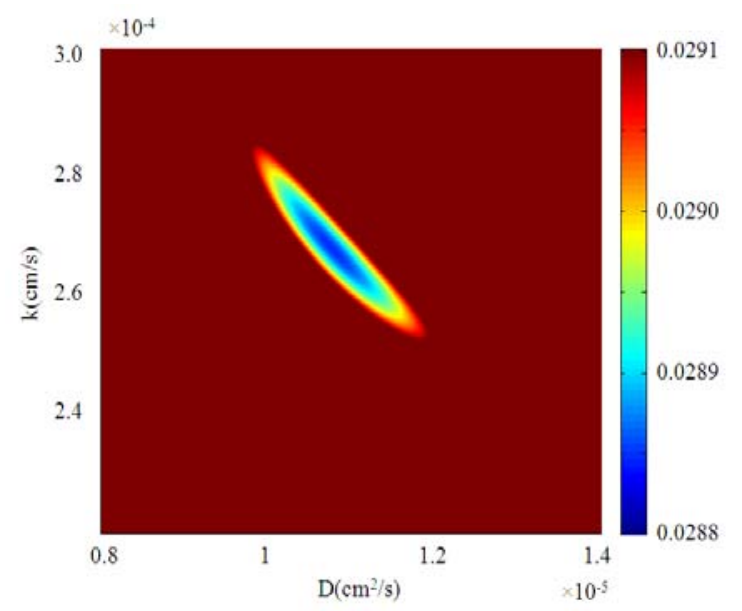

(h) $0.02 \mathrm{~atm} \rightarrow 0.05 \mathrm{~atm}$

Figure 7.6 Fitting error color maps for LSC under different oxygen partial pressure

Figure 7.6 is the fitting error color maps generated on $(D, k)$ plane. $(D, k)$ region was fitted within the $101 \%$ of the minimum value for the fitting error. Observation of the general trend revealed that both of the oxygen surface exchange and bulk diffusion coefficients decreased with the oxygen partial pressure. Parameter variations depending on $P_{\mathrm{O}_{2}}$ were plotted in Figure 7.6. The results showed that under our testing conditions the oxygen surface exchange coefficient for 
LSC still varies with the $P_{O_{2}}^{1 / 2}$ law as illustrated in Chapter 6. On the other side, the bulk diffusion coefficient possessed a linear relationship with oxygen partial pressure. It indicated that the atmosphere change will result in a more obvious effect for oxygen transport in LSC than LSCF.

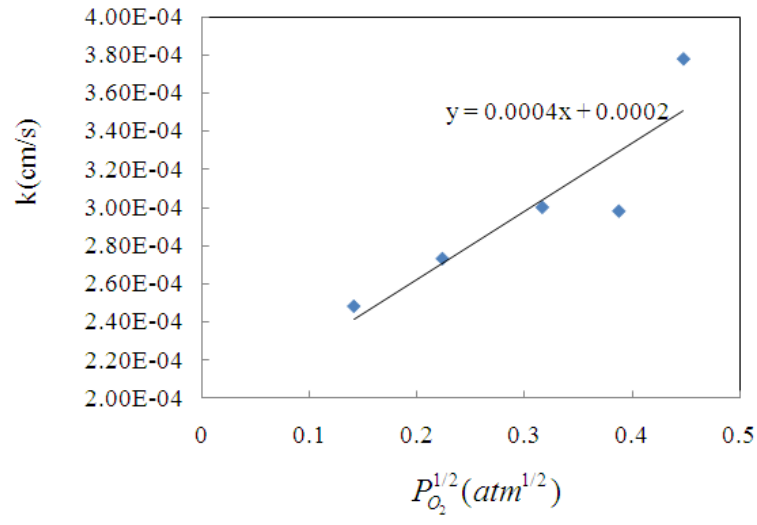

(a) Oxygen surface exchange coefficient

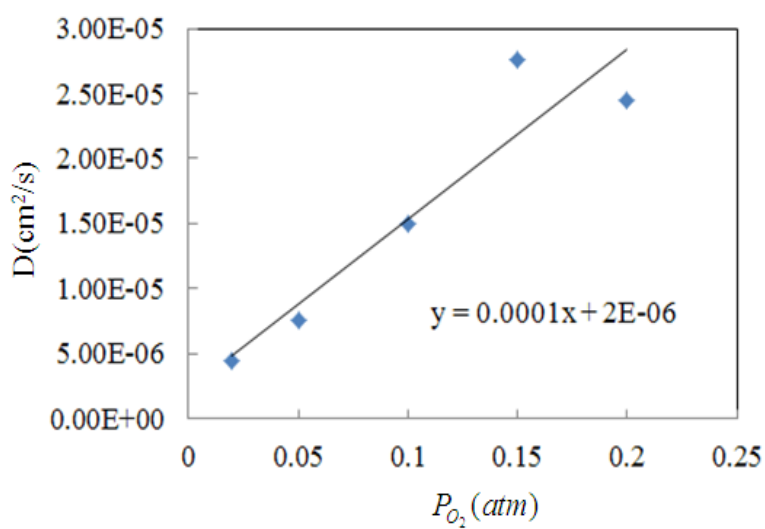

(b) Oxygen bulk diffusion coefficient

Figure 7.7 Oxygen transport kinetic parameters for $\mathrm{La}_{0.6} \mathrm{Sr}_{0.4} \mathrm{CoO}_{3-\delta}$ at $800^{\circ} \mathrm{C}$ as a function of oxygen partial pressure obtained from conductivity relaxation

\subsection{Oxygen transport behavior at infiltrated/backbone material interface}

7.4.1 Oxygen transport in the multilayer sample

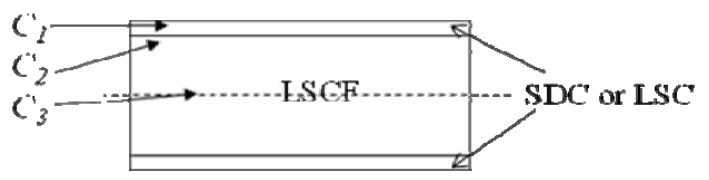

Figure 7.8 Sketch for the multilayer sample

Figure 7.8 is the cross section sketch for the spin coated SDC/LSCF or LSC/LSCF sample. We assumed that during the relaxation, the oxygen concentration in the infiltrated material, in LSCF close to the interface of SDC/LSCF/SDC or LSC/LSCF/LSC and in the middle of the multilayer sample are $C_{1}, C_{2}$ and $C_{3}$, respectively. And when the system obtains equilibrium under the new 
oxygen partial pressure, the saturated concentration are $C_{\infty}^{\prime}$ and $C_{\infty}$ separately for the infiltrated material and LSCF. Besides, net oxygen flux $\left(J,\left[\mathrm{~mol} / \mathrm{cm}^{2} \cdot \mathrm{s}\right]\right)$ was assumed as a constant, and the diffusion process inside the infiltrated layer was ignored due to its thickness. Oxygen transport can then be described with three equations listed below.

$$
\begin{aligned}
& C_{1}-C_{\infty}^{\prime}=\frac{J}{k_{\text {surface }}} \\
& C_{2}-C_{\infty}=\frac{J}{k_{\text {interface }}} \\
& C_{3}-C_{2}=\frac{J a}{D}
\end{aligned}
$$

Equation 7.3 is the surface exchange occurring on the gas/infiltrated material interface. Equation 7.4 represents the exchange occurring at the infiltrated material/LSCF interface and Equation 7.5 shows the diffusion inside LSCF. Therefore, the total surface exchange coefficient $(k)$ can be deduced by combining Equation 7.3 and 7.4.

$$
\frac{1}{k}=\frac{1}{k_{\text {interface }}}+\frac{1}{k_{\text {surface }}}
$$

$k_{\text {interface }}$ can be calculated from the experimentally obtained $k$ and $k_{\text {surface }}$ value. This parameter is pivotal for understanding the overall oxygen transport processes in the SOFC cathode and for further optimizing the infiltrated materials. The corresponding basic parameter values for substrate and spin coated materials are listed in Table 7.1.

Table 7.1 Basic oxygen transport kinetic parameters for simulation multilayer sample

\begin{tabular}{c|c|c|c|c}
\hline$P_{O_{2}}$ range $(\mathrm{atm})$ & $0.20 \sim 0.18$ & $0.15 \sim 0.14$ & $0.10 \sim 0.09$ & $0.05 \sim 0.018$ \\
\hline$D_{L S C F}\left(\mathrm{~cm}^{2} / \mathrm{s}\right)$ & $1.3 \times 10^{-5}$ & $1.0 \times 10^{-5}$ & $5.0 \times 10^{-6}$ & $5.0 \times 10^{-6}$ \\
\hline$k_{L S C}(\mathrm{~cm} / \mathrm{s})$ & $3.6 \times 10^{-4}$ & $3.0 \times 10^{-4}$ & $3.0 \times 10^{-4}$ & $2.5 \times 10^{-4}$ \\
\hline
\end{tabular}




\subsubsection{Oxygen transport at SDC/LSCF interface}

Figure 7.9 shows normalized conductivity relaxation for spin coated SDC sample at $800^{\circ} \mathrm{C}$ for both oxidation and reduction steps at different oxygen partial pressures together with the respective fitted curves. With the oxygen partial pressure decreasing, the relaxation time increased. The total surface exchange coefficients for the SDC/LSCF/SDC multilayer sample obtained from the fitted data are listed in Table 7.2. The results reveal that oxygen surface exchange was accelerated compared to the single phase LSCF sample. Besides, it's reported that GDC $\left(\mathrm{Ce}_{0.9} \mathrm{Gd}_{0.1} \mathrm{O}_{2-\delta}\right)$ which has similar structure to SDC, possesses low self-surface exchange rate as $1 \times 10^{-18} \mathrm{~cm} / \mathrm{s}^{83}$ at $0.05 \mathrm{~atm}$. Therefore, oxygen surface exchange of SDC can be considered much lower than $1.0 \times 10^{-4} \mathrm{~cm} / \mathrm{s}$ which is the total surface exchange coefficient of the SDC/LSCF/SDC sample obtained by ECR measurement. From the relation among oxygen exchange at infiltrated material surface $\left(k_{\text {surface }}\right)$, interface exchange $\left(k_{\text {interface }}\right)$ and total oxygen exchange coefficients $(k)$, as shown in equation 7.5 , it is obvious that $k$ should be smaller than

$k_{\text {surface. }}$ It is revealed that the oxygen surface exchange rate of the SDC layer was accelerated compared to the bulk material. 


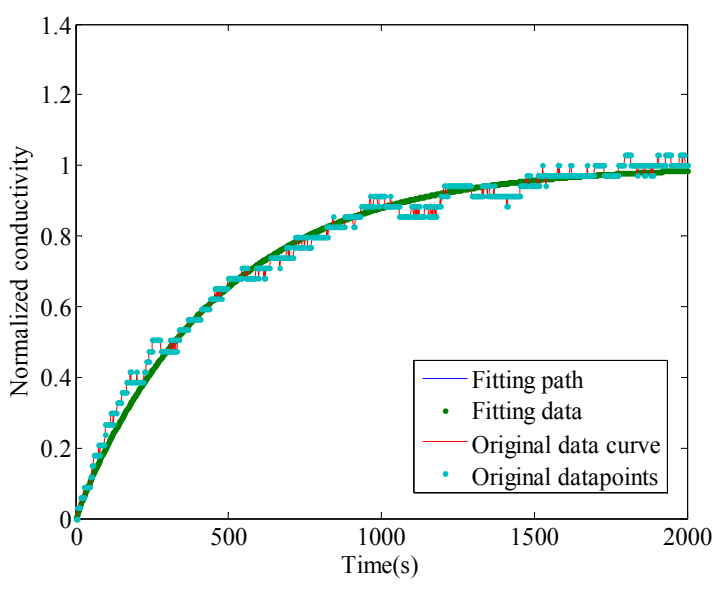

(a) $0.20 \mathrm{~atm} \rightarrow 0.18 \mathrm{~atm}$

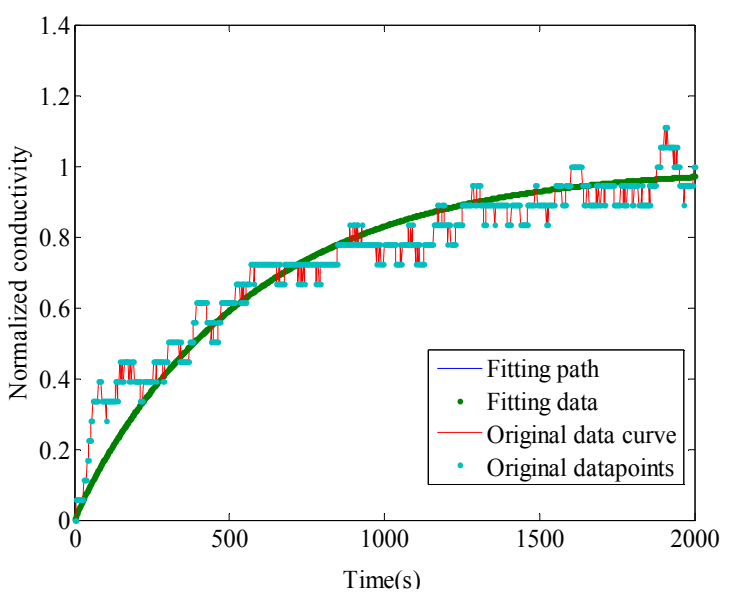

(c) $0.15 \mathrm{~atm} \rightarrow 0.14 \mathrm{~atm}$

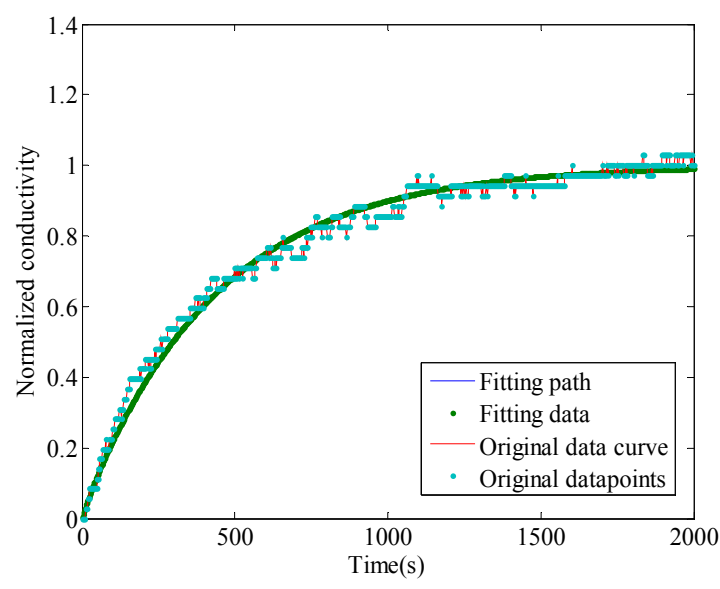

(b) $0.18 \mathrm{~atm} \rightarrow 0.20 \mathrm{~atm}$

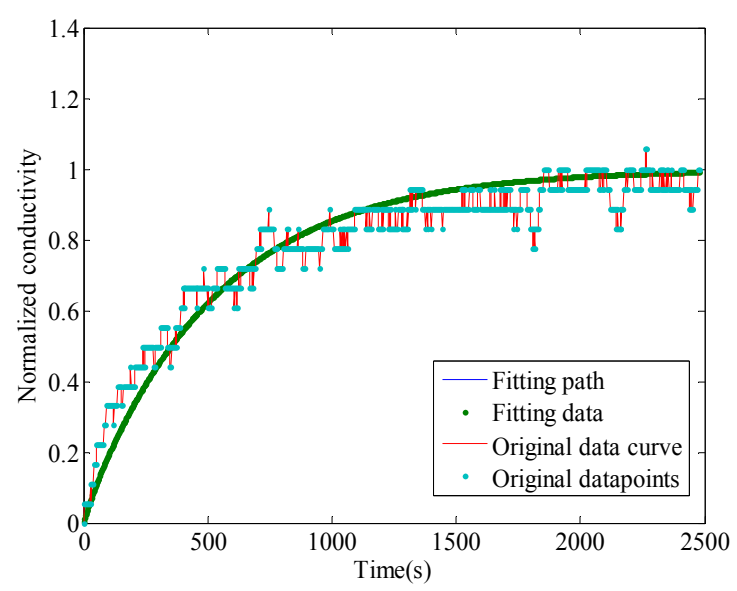

(d) $0.14 \mathrm{~atm} \rightarrow 0.15 \mathrm{~atm}$ 


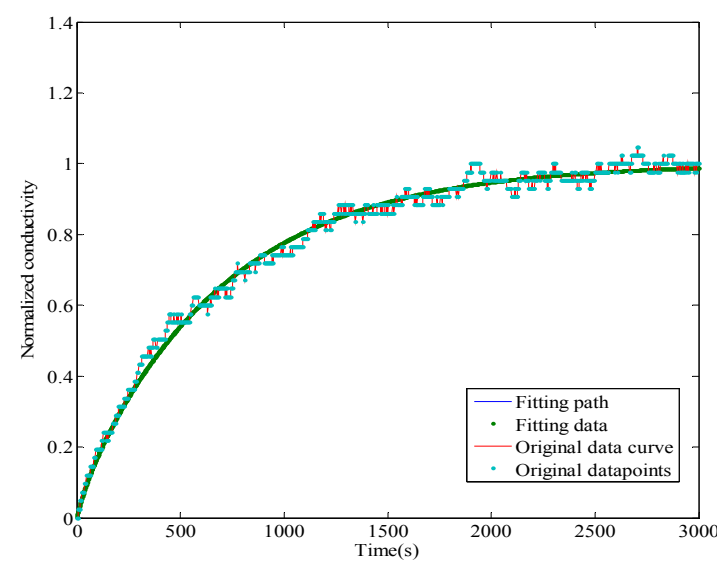

(e) $0.10 \mathrm{~atm} \rightarrow 0.09 \mathrm{~atm}$

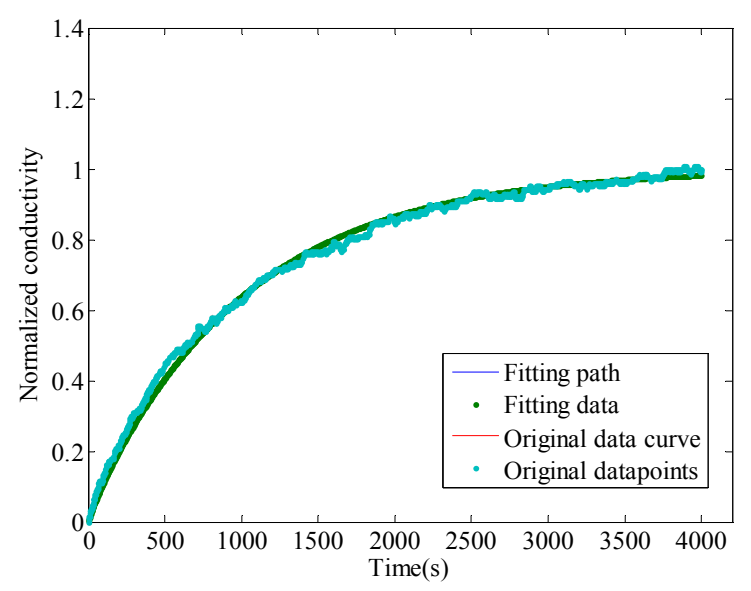

(g) $0.05 \mathrm{~atm} \rightarrow 0.04 \mathrm{~atm}$

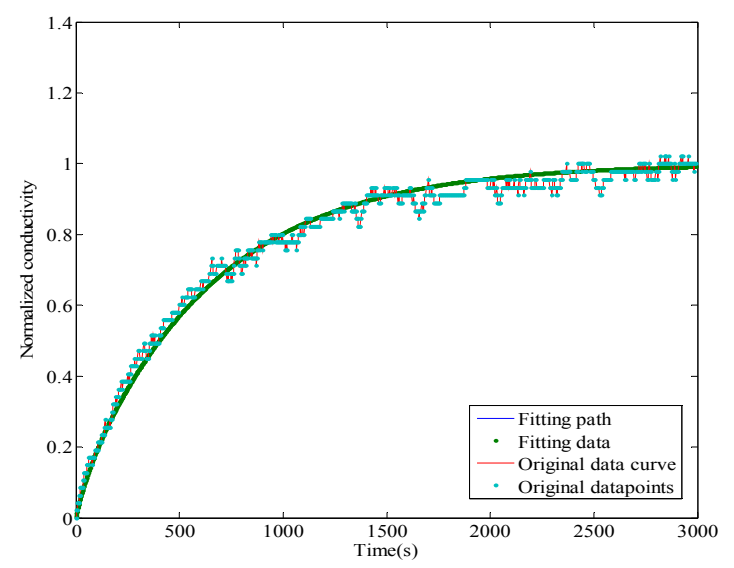

(f) $0.09 \mathrm{~atm} \rightarrow 0.10 \mathrm{~atm}$

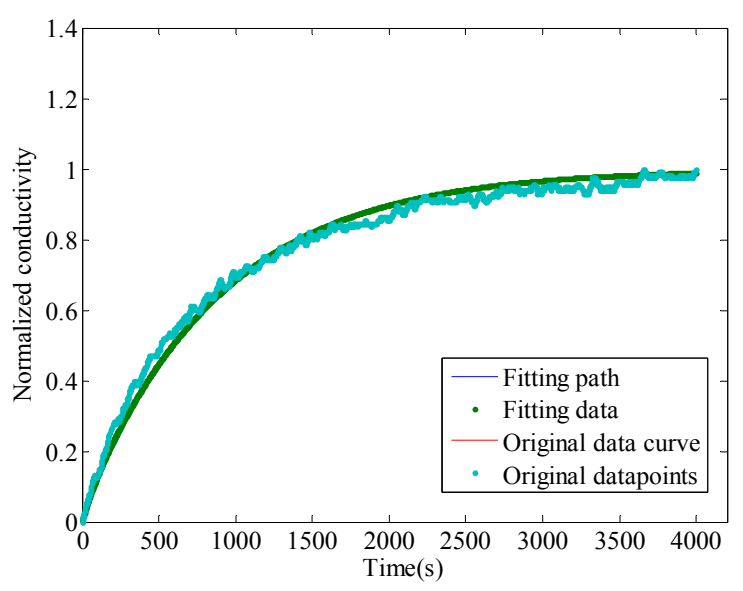

(h) $0.04 \mathrm{~atm} \rightarrow 0.05 \mathrm{~atm}$ 


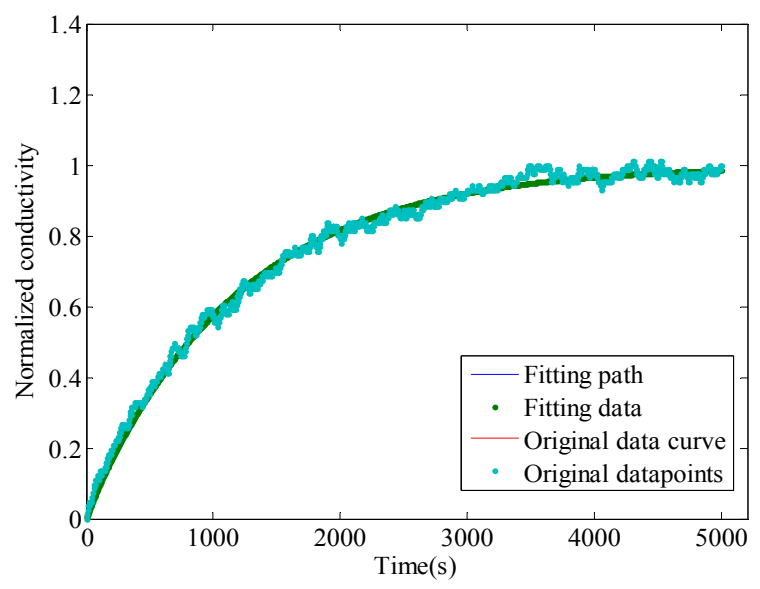

(i) $0.020 \mathrm{~atm} \rightarrow 0.018 \mathrm{~atm}$

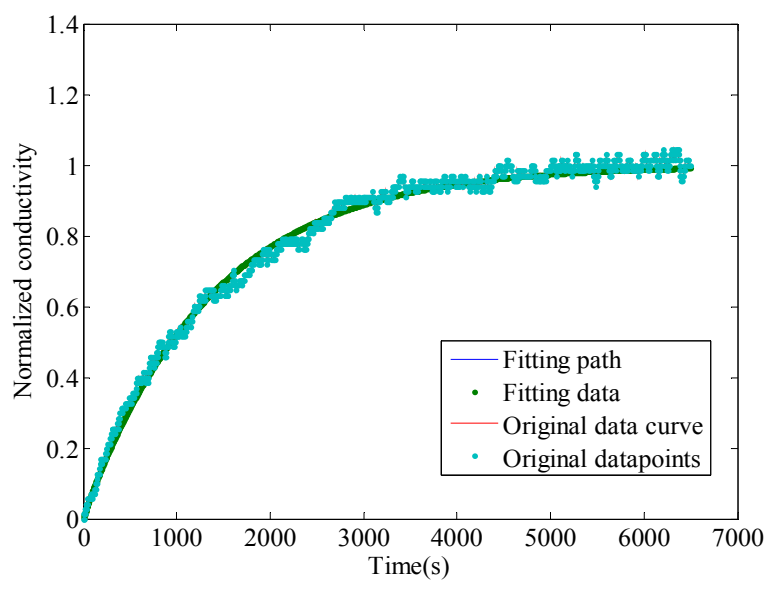

(j) $0.018 \mathrm{~atm} \rightarrow 0.020 \mathrm{~atm}$

Figure 7.9 Fitting results for SDC/LSCF/SDC multilayer sample

Table 7.2 Total oxygen surface exchange coefficient of spin coated SDC sample

\begin{tabular}{c|c|c|c|c|c}
\hline$P_{\mathrm{O}_{2}}(\mathrm{~atm})$ & 0.20 & 0.18 & 0.15 & 0.14 & 0.10 \\
\hline$k(\mathrm{~cm} / \mathrm{s})$ & $2.3 \times 10^{-4}$ & $2.0 \times 10^{-4}$ & $2.0 \times 10^{-4}$ & $1.8 \times 10^{-4}$ & $2.5 \times 10^{-4}$ \\
\hline$P_{O_{2}}(\mathrm{~atm})$ & 0.09 & 0.05 & 0.04 & 0.02 & 0.018 \\
\hline$k(\mathrm{~cm} / \mathrm{s})$ & $2.1 \times 10^{-4}$ & $1.3 \times 10^{-4}$ & $1.0 \times 10^{-4}$ & $6.8 \times 10^{-5}$ & $8.4 \times 10^{-5}$ \\
\hline
\end{tabular}

\subsubsection{Oxygen transport at LSC/LSCF interface}

Relaxation data in the form of normalized conductivity and corresponding fitting curves for spin coated LSC sample at $800^{\circ} \mathrm{C}$ under different oxygen partial pressures are shown in Figure 7.10. Total oxygen exchange coefficients are presented in Table 7.3. Considering the porous LSC layer, observed oxygen transport in spin coated LSC sample was faster than the spin coated SDC sample. To deduce oxygen exchange coefficient at LSC/LSCF interface, intrinsic oxygen surface exchange coefficient of LSC $\left(k_{L S C}\right)$ needs to be modified. 


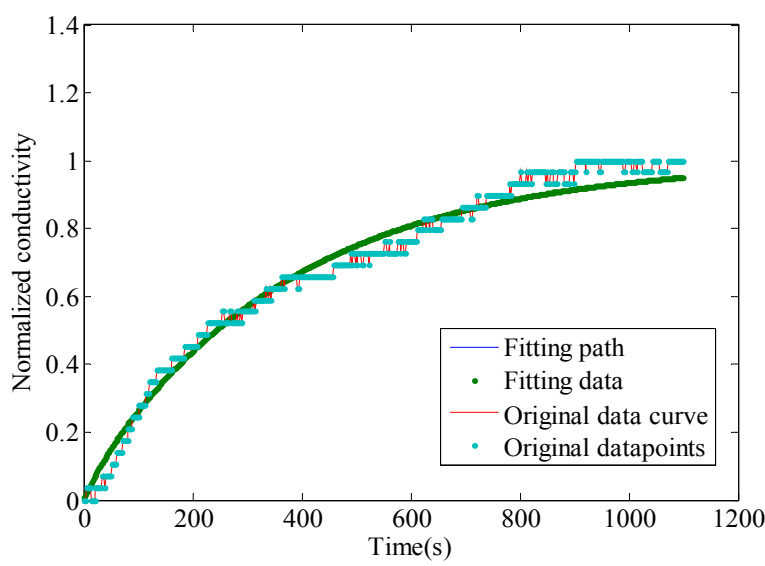

(a) $0.20 \mathrm{~atm} \rightarrow 0.18 \mathrm{~atm}$

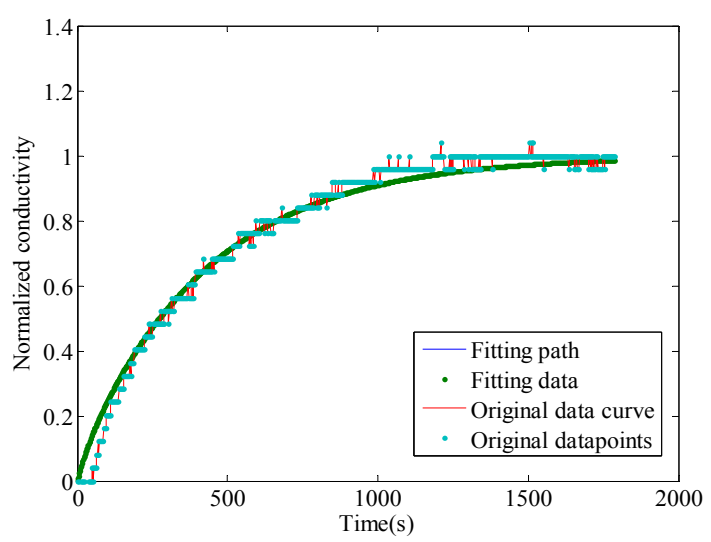

(c) $0.15 \mathrm{~atm} \rightarrow 0.14 \mathrm{~atm}$

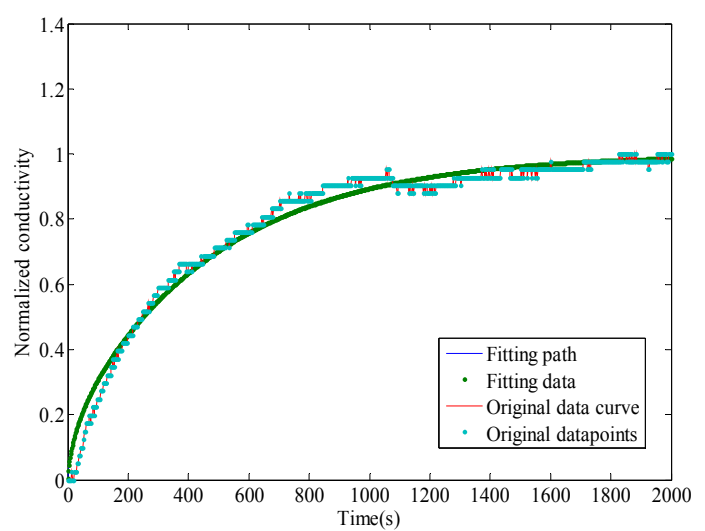

(e) $0.10 \mathrm{~atm} \rightarrow 0.09 \mathrm{~atm}$

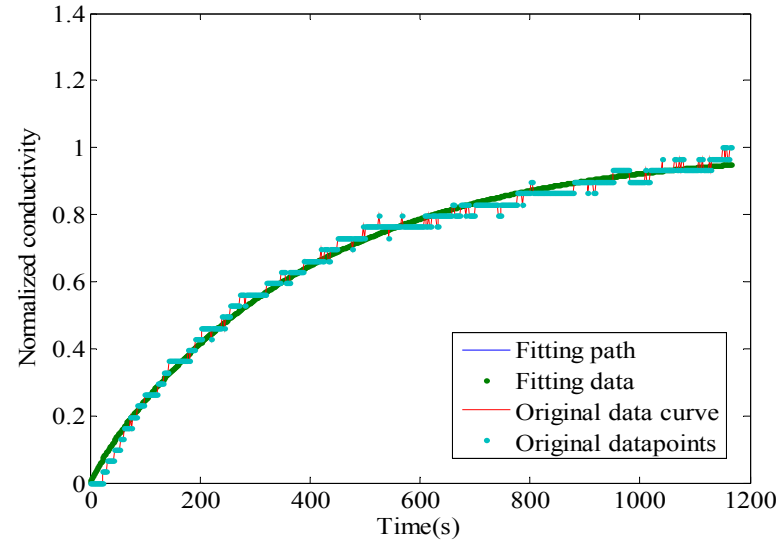

(b) $0.18 \mathrm{~atm} \rightarrow 0.20 \mathrm{~atm}$

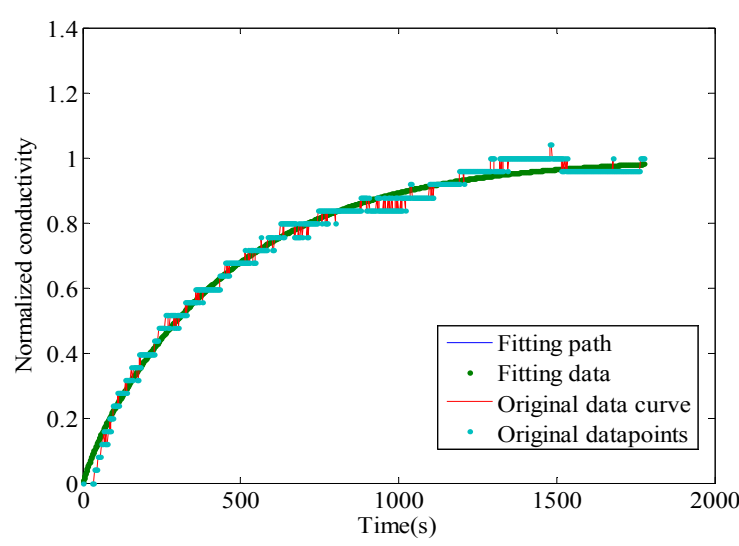

(d) $0.14 \mathrm{~atm} \rightarrow 0.15 \mathrm{~atm}$

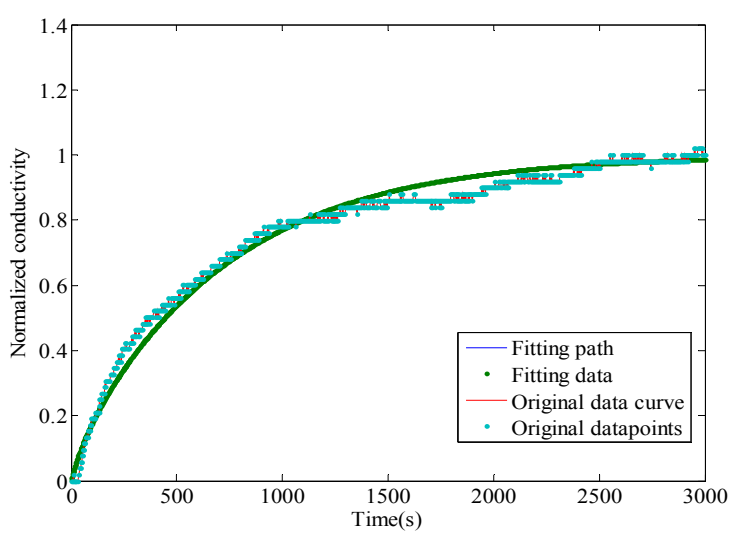

(f) $0.09 \mathrm{~atm} \rightarrow 0.10 \mathrm{~atm}$ 


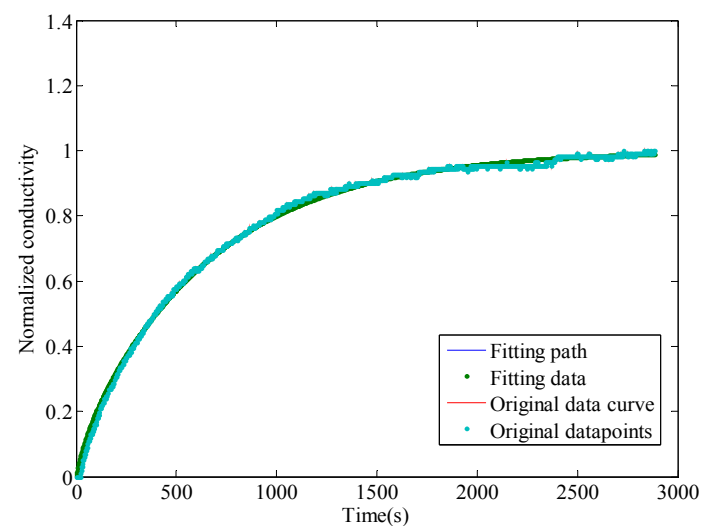

(g) $0.05 \mathrm{~atm} \rightarrow 0.04 \mathrm{~atm}$

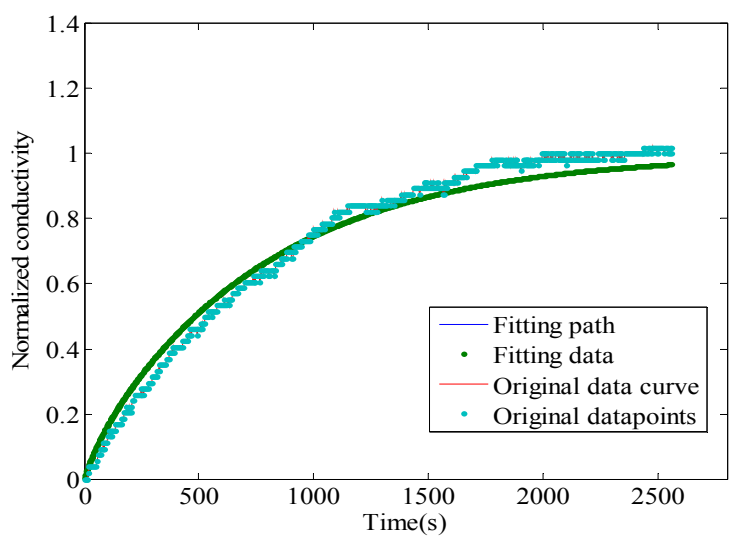

(i) $0.02 \mathrm{~atm} \rightarrow 0.018 \mathrm{~atm}$

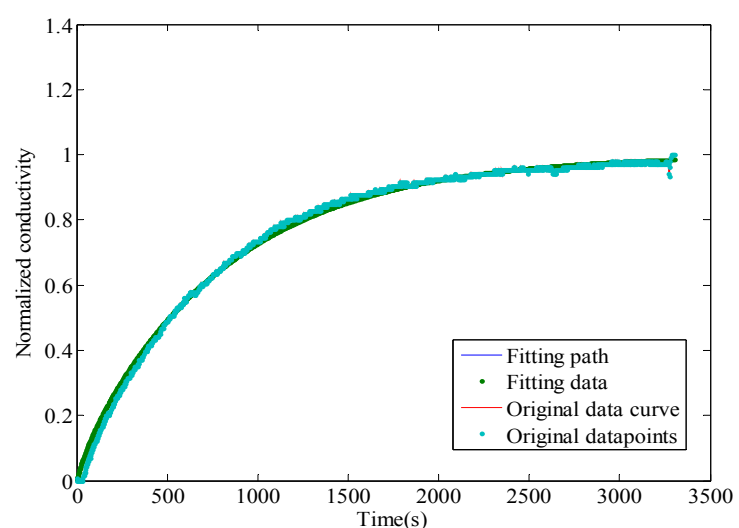

(h) $0.04 \mathrm{~atm} \rightarrow 0.05 \mathrm{~atm}$

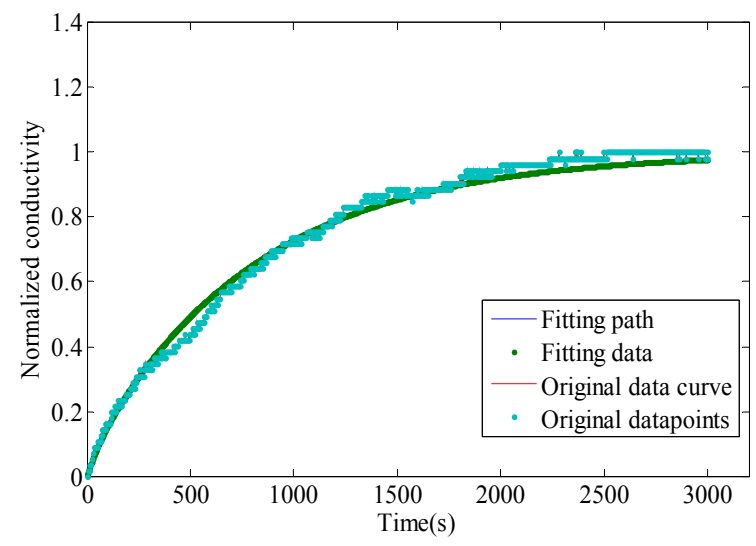

(j) $0.018 \mathrm{~atm} \rightarrow 0.02 \mathrm{~atm}$

Figure 7.10 Fitting results for LSC/LSCF/LSC multilayer sample

Table 7.3 Total oxygen surface exchange coefficient of spin coated LSC sample

\begin{tabular}{c|c|c|c|c|c}
\hline$P_{\mathrm{O}_{2}}(\mathrm{~atm})$ & 0.20 & 0.18 & 0.15 & 0.14 & 0.10 \\
\hline$k(\mathrm{~cm} / \mathrm{s})$ & $3.2 \times 10^{-4}$ & $3.5 \times 10^{-4}$ & $3.0 \times 10^{-4}$ & $3.5 \times 10^{-4}$ & $2.6 \times 10^{-4}$ \\
\hline$P_{O_{2}}(\mathrm{~atm})$ & 0.09 & 0.05 & 0.04 & 0.02 & 0.018 \\
\hline$k(\mathrm{~cm} / \mathrm{s})$ & $2.2 \times 10^{-4}$ & $1.9 \times 10^{-4}$ & $3.3 \times 10^{-4}$ & $1.9 \times 10^{-4}$ & $2.2 \times 10^{-4}$ \\
\hline
\end{tabular}


Based on LSC sample's testing results, for porous sample with an average particle size on the order of a few micrometers, the kinetics of oxygen transport is expected to be dictated exclusively by surface exchange. Relation among the average concentration at time $t(C(t)$, $\left.\left[\mathrm{mol} / \mathrm{cm}^{3}\right]\right)$, equilibrium concentration $\left(C(\infty),\left[\mathrm{mol} / \mathrm{cm}^{3}\right]\right)$ and oxygen surface exchange coefficient $\left(k_{L S C},[\mathrm{~cm} / \mathrm{s}]\right)$ is given by ${ }^{84}$ :

$$
S_{V} k_{L S C}[C(\infty)-C(t)] d t=\left(1-V_{V}\right) d C(t)
$$

$S_{V}$ is the specific surface area which stands by the fraction of pore surface to the total volume, $\left[\mathrm{cm}^{-1}\right] . V_{V}$ is the volume fraction porosity, [-]. Integrate equation 7.7:

$$
\frac{C(\infty)-C(t)}{C(\infty)-C(0)}=\exp \left[-\frac{t}{\left(\frac{1-V_{V}}{S_{V} k_{L S C}}\right)}\right]
$$

In terms of the normalized conductivity, equation 7.8 can be written as:

$$
\frac{\sigma(t)-\sigma(0)}{\sigma(\infty)-\sigma(0)}=\frac{C(t)-C(0)}{C(\infty)-C(0)}=1-\exp \left[-\frac{t}{\left(\frac{1-V_{V}}{S_{V} k_{L S C}}\right)}\right]
$$

Combined with equation 5.4, the extrinsic surface exchange coefficient for the porous layer can be given by:

$$
k_{\text {surf }}=\frac{c S_{V} k_{L S C}}{1-V_{V}}
$$

where $c$ is the thickness of the LSC layer, [cm].

Line interception was applied to estimate $S_{V}$ of porous LSC layer according to Virkar's work ${ }^{84}$. Intersection counts $P_{L}$ are determined from the cross section image and its value is $0.35 / \mu \mathrm{m}$. Specific surface area $S_{V}$ can be given by: 


$$
S_{V}=2 P_{L}
$$

The porosity $V_{V}$ is given by:

$$
V_{V}=\frac{\sum_{i=1}^{N} n_{i} r^{2} \pi}{A}
$$

$A$ is the detected area of the porous layer, $\left[\mu \mathrm{m}^{2}\right] . n$ is the number of certain size pores and $r$ is the diameter of those pores, $[\mu \mathrm{m}]$. The porosity of the LSC layer calculated is $28.3 \%$.

Deduced results are listed in Table 7.4 and it shows the oxygen exchange rates at LSC/LSCF interface are close to the rates on LSC surface.

Table 7.4 Oxygen interface exchange coefficient of spin coated LSC sample

\begin{tabular}{c|c|c|c|c|c}
\hline$P_{O_{2}}(\mathrm{~atm})$ & 0.20 & 0.18 & 0.15 & 0.14 & 0.10 \\
\hline$k(\mathrm{~cm} / \mathrm{s})$ & $3.4 \times 10^{-4}$ & $3.8 \times 10^{-4}$ & $3.3 \times 10^{-4}$ & $3.9 \times 10^{-4}$ & $2.8 \times 10^{-4}$ \\
\hline$P_{O_{2}}(\mathrm{~atm})$ & 0.09 & 0.05 & 0.04 & 0.02 & 0.018 \\
\hline$k(\mathrm{~cm} / \mathrm{s})$ & $2.3 \times 10^{-4}$ & $2.0 \times 10^{-4}$ & $3.7 \times 10^{-4}$ & $2.0 \times 10^{-4}$ & $2.4 \times 10^{-4}$ \\
\hline
\end{tabular}




\subsubsection{Discussion}

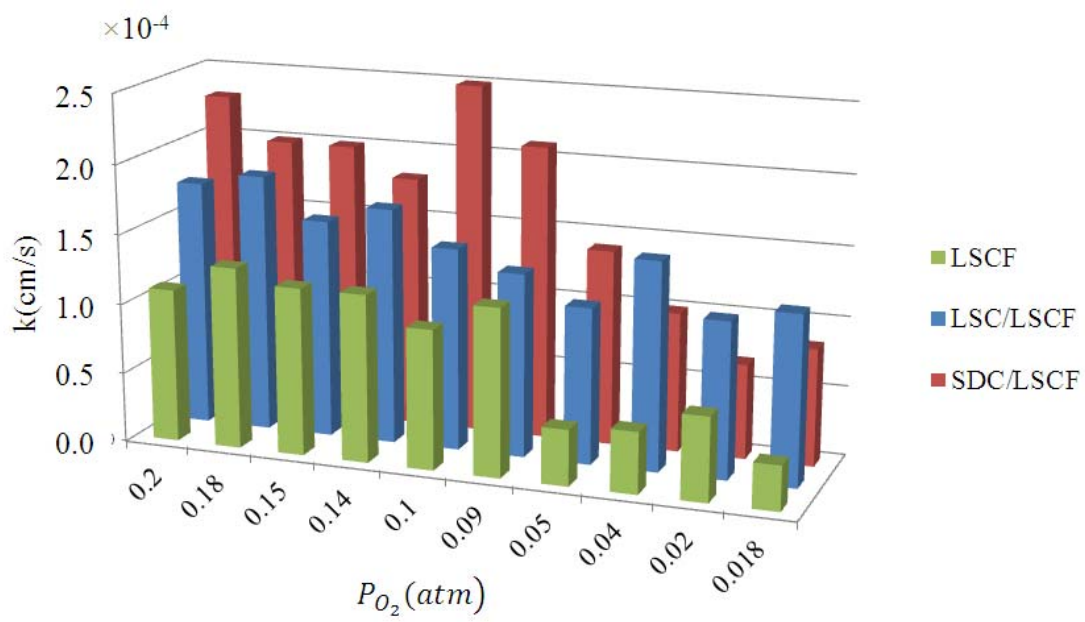

Figure 7.11 Surface exchange coefficients of single phase LSCF and infiltrated cathodes

Two composite cathode materials including LSC infiltrated LSCF and SDC infiltrated LSCF have been investigated in this chapter. Due to the difficulty of characterizing SDC by ECR testing, only the total surface exchange coefficient has been observed for SDC infiltrated LSCF. With the deduced oxygen exchange coefficient at LSC/LSCF interface, the total surface exchange coefficient of LSCF substrate with dense LSC film could be simulated. Corresponding results are summarized in Figure 7.11. The results offered a quantitative description of the promotion by infiltration and indicated that both of the infiltrated materials improved oxygen reduction rate compared to single phase LSCF material. Under high $P_{\mathrm{O}_{2}}$, oxygen surface exchange of SDC infiltrated LSCF is faster than LSC infiltrated LSCF. The advantage of SDC infiltrated LSCF material disappeared when oxygen partial pressure was under 0.04atm.

The experimental results can be summarized by two key points: 
(1) Oxygen reduction will occur at the surface of the infiltrated material instead of cathode backbone. The reduced oxygen ion will then be transferred into LSCF at infiltrated material/LSCF interface. Oxygen ion interface exchange is similar to the diffusion process. It is suspected to be easier than reducing molecular oxygen to oxygen ions, which requires cleavage of a chemical bond. Therefore, the interface exchange coefficients for the spin coated samples are higher than oxygen surface exchange coefficient of LSCF.

(2) The surface exchange process is considered controlled by oxygen partial pressure, and the electron and oxygen vacancy concentrations. Oxygen capture activity of SDC has been improved with spin coated LSCF. It may be because the electron activity was promoted through the substrate and the thin SDC layer. At high $P_{\mathrm{O}_{2}}$, the SDC infiltrated LSCF cathode was expected to show higher performance than the LSC infiltrated one. However, when the oxygen partial pressure decreased, total surface exchange coefficient of LSC/LSCF/LSC sample became bigger than SDC/LSCF/SDC. It may be caused by the different interface structure of the two infiltrated materials. The similar crystal structure of LSC and LSCF may result in easier oxygen vacancy exchange at their interface.

\subsection{Concluding remarks}

Electrical conductivity relaxation has been developed to investigate infiltrated cathode materials in this chapter. Widely used infiltrated materials $\mathrm{Ce}_{0.8} \mathrm{Sm}_{0.2} \mathrm{O}_{1.9}$ and $\mathrm{La}_{0.6} \mathrm{Sr}_{0.4} \mathrm{CoO}_{3-\delta}$ were chosen as the research objectives. Oxygen transport behavior of the infiltrated materials has been studied at first. Multilayer samples prepared by spin coating LSC or SDC on LSCF substrate were applied for ECR measurement. With the experimentally obtained oxygen kinetic parameters for infiltrated and cathode backbone materials, oxygen exchange coefficients on the 
infiltrate/cathode backbone interface had been deduced. The results revealed that both the SDC infiltrated and LSC infiltrated cathode materials possess higher oxygen surface exchange rate compared to single phase LSCF material. Besides, oxygen surface exchange of SDC infiltrated LSCF is faster than LSC infiltrated LSCF under high oxygen partial pressure. 


\section{Fundamental understanding for infiltrated SOFC cathode}

\subsection{Introduction}

Oxygen transport in SOFC cathodes has been investigated extensively. However, most of the present modeling work is based on single phase cathode material. Computational approaches on infiltrated cathode have been rarely reported. In this chapter, we will use a micro-model to analyze the oxygen reduction mechanism on an infiltrated cathode. Key to our model is treatment for the new interface formed between infiltrate/cathode backbone materials. Charge transfer across this new interface was assumed as the rate limiting step when using mixed ionic and electronic conductor for the cathode backbone. Simulation results were generated under low cathode over-potential at equilibrium state. This model is generally valid for an infiltrated material with high oxygen surface exchange activity.

\subsection{Physical model and basic reaction steps}

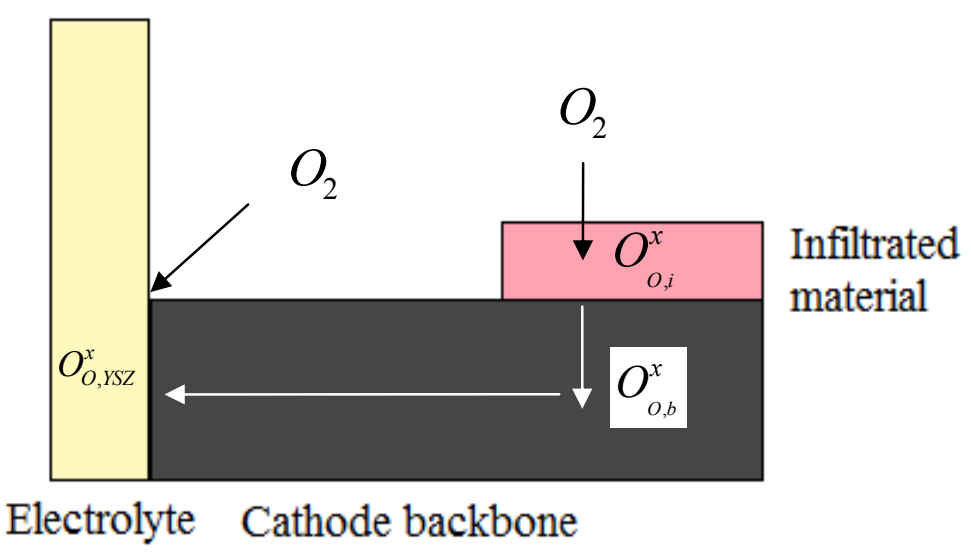

Figure 8.1 Sketch for infiltrated cathode model

The infiltrated cathode model is depicted in Figure 8.1. A dense SOFC electrolyte material such as yttria stabilized zirconia (YSZ) contacts a mixed ionic and electronic conductor such as LSCF. 
High oxygen reduction activity materials such as SDC or LSC are infiltrated on top of the LSCF to form a non-continuous layer. To make a clear oxygen transport pathway only one infiltrated nano particle was shown in Figure 8.1. The cathode backbone/electrolyte interface is taken to be $x=0$. Two pathways are assumed to exist. The first one is through traditional oxygen reduction zone, three phase boundary (3PB) where gas, cathode and electrolyte meet. Charge transfer across cathode backbone/electrolyte interface will be considered as the second possible pathway.

In the development of this infiltrated cathode model, we make several assumptions and limitations which are summarized below.

(1) Infiltrated nano particles will cover most surfaces of the backbone cathode but will not form a continuous network and contact the electrolyte.

(2) The cathode backbone has high electronic conductivity and also exhibits sufficient bulk conductivity for oxygen ions.

(3) Oxygen reduction occurring at the gas/infiltrated material surface is considered a chemical reaction due to the high oxygen reduction catalytic property of the infiltrated material.

(4) The equilibrium state of the adsorbed surface coverage is expressed by the Langmuir isotherm, assuming that rate constants are independent of concentration and that adsorption occurs directly with no precursor states.

(5) Since the cathode backbone material possesses high electronic conductivity, the potential is locally uniform through the thickness of the cathode.

(6) The electrolyte is treated as ohmic and of unity ionic transference.

(7) It is assumed that no drop in the oxygen partial pressure occurs along the cathode surface as a result of the oxygen reduction reaction. 
With all the limitations and assumptions outlined above, the oxygen reduction reaction can be described using the following kinetic description. For the 2PB pathway, gaseous oxygen is assumed to undergo adsorption/desorption at the infiltrated material surface. The adsorbed oxygen will then be reduced to oxygen ions in the infiltrated material due to its high catalytic activity towards oxygen reduction. Once transferred into the cathode backbone, the oxygen ion will diffuse to the electrode/electrolyte interface. By reaction 8.4, the oxygen ion will enter YSZ with direct vacancy exchange.

$$
\begin{gathered}
O_{2}+s \underset{k_{1}^{-}}{\stackrel{k_{1}}{\rightleftarrows}} O_{2, a d} \\
O_{2, a d}+2 V_{O, I}^{\ddot{k_{2}}} \underset{k_{2}^{-}}{\stackrel{k_{2}}{\rightleftarrows}} O_{o, I}^{x}+4 h+s \\
O_{O, I}^{x}+V_{O, L S C F}^{\ddot{k_{3}^{-}}} V_{O, I}+O_{O, L S C F}^{x} \\
O_{O, L S C F}^{x}+V_{\ddot{O}, Y S Z}^{\stackrel{k_{4}}{\rightleftarrows}} O_{k_{4}^{-}}^{x}
\end{gathered}
$$

At the 3PB region, since the infiltrated material is assumed not in contact with the electrolyte, oxygen will be adsorbed on the cathode backbone surface and then be reduced. However, considering the infiltration catalytic promotion and the effective traditional TPB area, oxygen flow at the 3PB will be ignored in this work.

$$
\begin{gathered}
O_{2}+s \underset{k_{5}^{-}}{\stackrel{k_{5}}{\rightleftarrows}} O_{2, a d, L S C F} \\
O_{2, a d, L S C F}+2 V_{O, Y S Z}^{\stackrel{k_{6}}{\rightleftarrows}} 2 O_{o, Y S Z}^{x}+4 h^{-}+s
\end{gathered}
$$

The vacancy exchange occurring at the electrode/electrolyte interface, as shown in Equation 8.4, is treated as an electrochemically activated process and its rate is presented by the Butler-Volmer 
equation. On the other hand, oxygen adsorption and reduction reactions at the infiltrated material surface are considered under chemical control since they possess high oxygen reduction catalytic properties. Moreover, the oxygen ion exchange at infiltrated/ cathode backbone interface (Equation 8.3) is also considered as chemical force motivated for there no net charger transfer involved. The rates of oxygen transport are then given by:

$$
\begin{gathered}
r_{1}=k_{1} P_{O_{2}} \Gamma(1-\theta)-k_{1}^{-} \Gamma \theta \\
r_{2}=k_{2} \Gamma \theta\left(C_{V, I}\right)^{2}-k_{2}^{-} \Gamma(1-\theta) \\
r_{3}=k_{3} C_{V, L S C F}-k_{3}^{-} C_{V, I} \\
r_{4}=k_{4} C_{V, Y S Z} \exp \left(-2 \alpha_{2 P B} f \eta\right)-k_{4}^{-} C_{V, L S C F} \exp \left[2\left(1-\alpha_{2 P B}\right) f \eta\right]
\end{gathered}
$$

$\Gamma$ is the surface oxygen adsorption site density, $\left[\mathrm{mol} / \mathrm{cm}^{2}\right] . \theta$ is a unit-less parameter describing the degree of coverage of adsorbed oxygen. $C_{V, Y S Z}, C_{V, I}$ and $C_{V, L S C F}$ indicate the oxygen vacancy concentration in the electrolyte, infiltrated and cathode backbone material, $\left[\mathrm{mol} / \mathrm{cm}^{3}\right] . \eta$ stands for the cathode over-potential, $[\mathrm{mV}]$. In addition, $\alpha_{2 P B}$ is the symmetry factor for the oxygen vacancy exchange reaction.

\subsection{Flux formulations and boundary conditions}

By assuming the infiltrated material possesses high oxygen reduction activity, $C_{V, I}$ can be treated as a constant. Correspondingly, the surface adsorption reaction for infiltrated material is assumed to retain a stable equilibrium state. Therefore, there is only one variable in this model, which is the oxygen vacancy concentration inside LSCF. Using Fick's diffusion laws and assuming steady-state flux, the vacancy transport can be described as:

$$
\frac{\partial C_{V, L S C F}}{\partial t}=D \frac{\partial^{2} C_{V, L S C F}}{\partial x^{2}}-\frac{\Delta S}{\Delta V} r_{3}
$$


$\Delta \mathrm{S} / \Delta \mathrm{V}$ is the volume-specific area of the cathode backbone, $\left[\mathrm{cm}^{-1}\right]$.

To solve this differential equation, two boundary conditions are defined. At $x=0$, the flux of oxygen vacancy is related to vacancy exchange at the electrolyte/cathode interface.

$$
D \frac{\partial C_{V, L S C F}}{\partial x}=-r_{4}
$$

At the outer surface of the cathode, $x=l_{c}$, net flux of oxygen vacancy should equal zero. In other words, beyond this point, oxygen bulk diffusion will become the rate limiting step instead of the oxygen interface exchange.

$$
\frac{d C_{V, L S C F}}{d x}=0
$$

\subsection{Potential and current-potential relationship}

A three electrode system can be applied to characterize the infiltrated cathode system. Platinum paste current collectors contacting the outer surfaces of the cathode and the electrolyte function as working electrode (WE) and counter electrode (CE) separately. A Pt wire reference electrode is buried inside the electrolyte.

The total open circuit potential yields Nernst equation:

$$
E_{\text {OCV }}=-\frac{R T}{4 F} \ln \frac{P_{O_{2}}^{R E}}{P_{O_{2}}^{W E}}
$$

The potential drop across cathode/electrolyte interface can be given by:

$$
E_{2 P B}=E_{2 P B}^{o c}+\eta
$$

Since reaction 8.4 is the electrochemical activated step subject to Butler-volmer treatment, the current across cathode/electrolyte then becomes: 


$$
\begin{aligned}
& i_{4}=i_{4,0}\left[\exp \left(-2 \alpha_{2 P B} f \eta\right)-\frac{C_{V, L S C F}}{C_{V, L S C F, e q}} \exp \left[2\left(1-\alpha_{2 P B}\right) f \eta\right]\right. \\
& C_{V, L S C F, e q}=\frac{k_{4}}{k_{4}^{-}} C_{V, Y S Z} \exp (-2 f \eta)
\end{aligned}
$$

If reaction 8.3 is the rate limited step, the adsorption and reduction reactions occurring on infiltrated material will achieve equilibrium. In other words, the net reaction rate of 8.1 and 2 should equal to zero. Then the relationship between oxygen vacancy concentration inside the infiltrated material and oxygen partial pressure can be deduced:

$$
C_{V, I}=\left(\frac{k_{1}^{-} k_{2}^{-}}{k_{1} k_{2} P_{O_{2}}}\right)^{1 / 2}
$$

Furthermore the exchange current can be given by:

$$
r_{3,0}=k_{3}^{-} C_{V, I}=k_{3}^{-}\left(\frac{k_{1}^{-} k_{2}^{-}}{k_{1} k_{2} P_{O_{2}}}\right)^{1 / 2}
$$

\subsection{Application of electrical conductivity relaxation results}

Oxygen ions exchanging at the infiltrate/cathode backbone material can be expressed by equation 8.3.

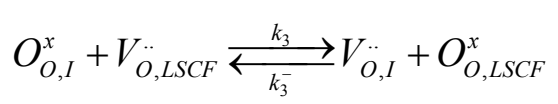

Considering the theoretical basis of the diffusion model, oxygen interface exchange coefficient and the reaction constants have the following relation.

$$
k_{\text {interface }}\left(C_{V, L S C F}-C_{V, L S C F, e q}\right)=k_{3} C_{V, L S C F}-k_{3}^{-} C_{V, I}
$$

By transforming, a further relation between $k_{\text {interface }}$ and $k_{3}^{-}$was obtained. 


$$
\begin{aligned}
& k_{\text {interface }}=\frac{k_{3} C_{V, L S C F}-k_{3}^{-} C_{V, I}}{C_{V, L S C F}-C_{V, L S C F, e q}} \\
& =\frac{k_{3}^{-} C_{V, I} \frac{C_{V, L S C F}}{C_{V, L S C F, e q}}-k_{3}^{-} C_{V, I}}{C_{V, L S C F}-C_{V, L S C F, e q}} \\
& =\frac{k_{3}^{-} C_{V, I}}{C_{V, L S C F, e q}}
\end{aligned}
$$

Therefore, the equilibrium exchange reaction rate $r_{3,0}$ can be estimated with the experimentally measured oxygen interface exchange coefficient and oxygen vacancy equilibrium concentration.

$$
r_{3,0}=k_{3} C_{V, L S C F, e q}=k_{3}^{-} C_{V, I}=k_{\text {interface }} C_{V, L S C F, e q}
$$

\subsection{Simulation results and discussion}

Table 8.1 Parameter values applied for simulation

\begin{tabular}{c|l|c|c}
\hline Parameter & Description & Units & Value \\
\hline$D$ & Bulk oxygen diffusivity of LSCF & $\mathrm{cm}^{2} / \mathrm{s}$ & $5 \times 10^{-6}$ \\
\hline$\Gamma$ & Surface adsorption site density & $\mathrm{mol} / \mathrm{cm}^{2}$ & $1 \times 10^{-9}$ \\
\hline$\theta$ & $\begin{array}{l}\text { Degree of coverage of adsorbed } \\
\text { oxygen }\end{array}$ & & 0.01 \\
\hline$\alpha_{2 P B}$ & Symmetry factor & $\mathrm{mol} / \mathrm{cm}^{3}$ & 0.5 \\
\hline$C_{V, I}$ & $\begin{array}{l}\text { Vacancy concentration in infiltrated } \\
\text { material }\end{array}$ & $\mathrm{mol} / \mathrm{cm}^{3}$ & $5 \times 10^{-4}$ \\
\hline$C_{V, Y S Z}$ & Vacancy concentration in YSZ & $\mathrm{mol} / \mathrm{cm}^{3}$ & variation \\
\hline$C_{V, L S C F}$ & Vacancy concentration in LSCF & $\mathrm{mol} / \mathrm{cm}^{3}$ & variation \\
\hline$C_{O, L S C F}$ & Oxygen ion concentration in LSCF & $\mathrm{mol} / \mathrm{cm}^{3}$ & $1 \times 10^{-4}$ \\
\hline$C_{V, L S C F, e q}$ & $\begin{array}{l}\text { Equilibrium vacancy concentration } \\
\text { in LSCF }\end{array}$ & & \\
\hline
\end{tabular}




\begin{tabular}{c|l|c|c}
\hline$\Delta S / \Delta V$ & Volume specific surface area & $\mathrm{cm}^{-1}$ & $6 \times 10^{5}$ \\
\hline $\mathrm{T}$ & Temperature & $\mathrm{K}$ & 1073 \\
\hline $\mathrm{r}_{1,0}$ & $\begin{array}{l}\text { Exchange current density for } \\
\text { Reaction 8.1 }\end{array}$ & $\mathrm{mol} / \mathrm{cm}^{2} \cdot \mathrm{s}$ & $1 \times 10^{-7}$ \\
\hline $\mathrm{r}_{2,0}$ & $\begin{array}{l}\text { Exchange current density for } \\
\text { Reaction 8.2 }\end{array}$ & $\mathrm{mol} / \mathrm{cm}^{2} \cdot \mathrm{s}$ & $1.5 \times 10^{-7}$ \\
\hline $\mathrm{r}_{3,0}$ & $\begin{array}{l}\text { Exchange current density for } \\
\text { Reaction 8.3 }\end{array}$ & $\mathrm{mol} / \mathrm{cm}^{2} \cdot \mathrm{s}$ & $1 \times 10^{-8} \sim 5 \times 10^{-8}$ \\
\hline $\mathrm{r}_{4,0}$ & $\begin{array}{l}\text { Exchange current density for } \\
\text { Reaction 8.4 }\end{array}$ & $\mathrm{A} / \mathrm{cm}^{2}$ & 0.2 \\
& $\mathrm{~mol} / \mathrm{cm}^{2} \cdot \mathrm{s}$ & $1 \times 10^{-6}$ \\
\hline
\end{tabular}

A finite control-volume analysis with time-discretization was applied to obtain the transient solution of the variables. The electrode distance is from the cathode/electrolyte interface $(x=0 \mathrm{~m})$ to $x=15 \mu \mathrm{m}$ and is divided into 40 flux nodes with equal spacing. The computational code is implemented with Visual $\mathrm{C}++$ 6.0. And parameter values applied in the simulation are listed in Table 8.1.

\subsubsection{Infiltrated material effects}

Figure 8.2 shows the simulated oxygen vacancy variation with different equilibrium oxygen exchange rate at the infiltrate/cathode backbone interface under $-0.2 \mathrm{~V}$ over-potential. Based on $E C R$ testing results for SDC and LSC infiltrated LSCF cathodes, $r_{3,0}$ value was fixed varying from $1 \times 10^{-8} \mathrm{~mol} / \mathrm{cm}^{2} \cdot \mathrm{s}$ to $5 \times 10^{-8} \mathrm{~mol} / \mathrm{cm}^{2} \cdot \mathrm{s}$. The active zone length for oxygen surface exchange was decreased with $r_{3,0}$ value increasing. It is caused by the fact that oxygen diffusivity is a constant while the surface exchange rate of the cathode backbone has been improved. $\left(l_{c}=D / k\right)$ On the other hand, current density at the cathode/electrolyte $2 \mathrm{~PB}$ interface can be considered as a result of the oxygen vacancy flux. And it is given by ${ }^{85}$ : 


$$
i_{2 P B}=i_{4}=\left.2 F D_{\text {backbone }}\left(\frac{\partial C_{V, M I E C}}{\partial x}\right)\right|_{x=0}
$$

The slope of the oxygen vacancy concentration curve at the cathode/electrolyte interface (electrode distance $=x=0 \mathrm{~m}$ ) is utilized to estimate $2 \mathrm{~PB}$ current density. From Figure 8.2, the oxygen vacancy concentration at $x=0$ is higher with smaller $r_{3,0}$. Based on equation 8.16 , the 2PB current should increase with the oxygen vacancy concentration decreasing under the same over-potential. However, this trend is not obvious here and it's due to the high equilibrium oxygen vacancy concentration of the cathode backbone (LSCF type material).

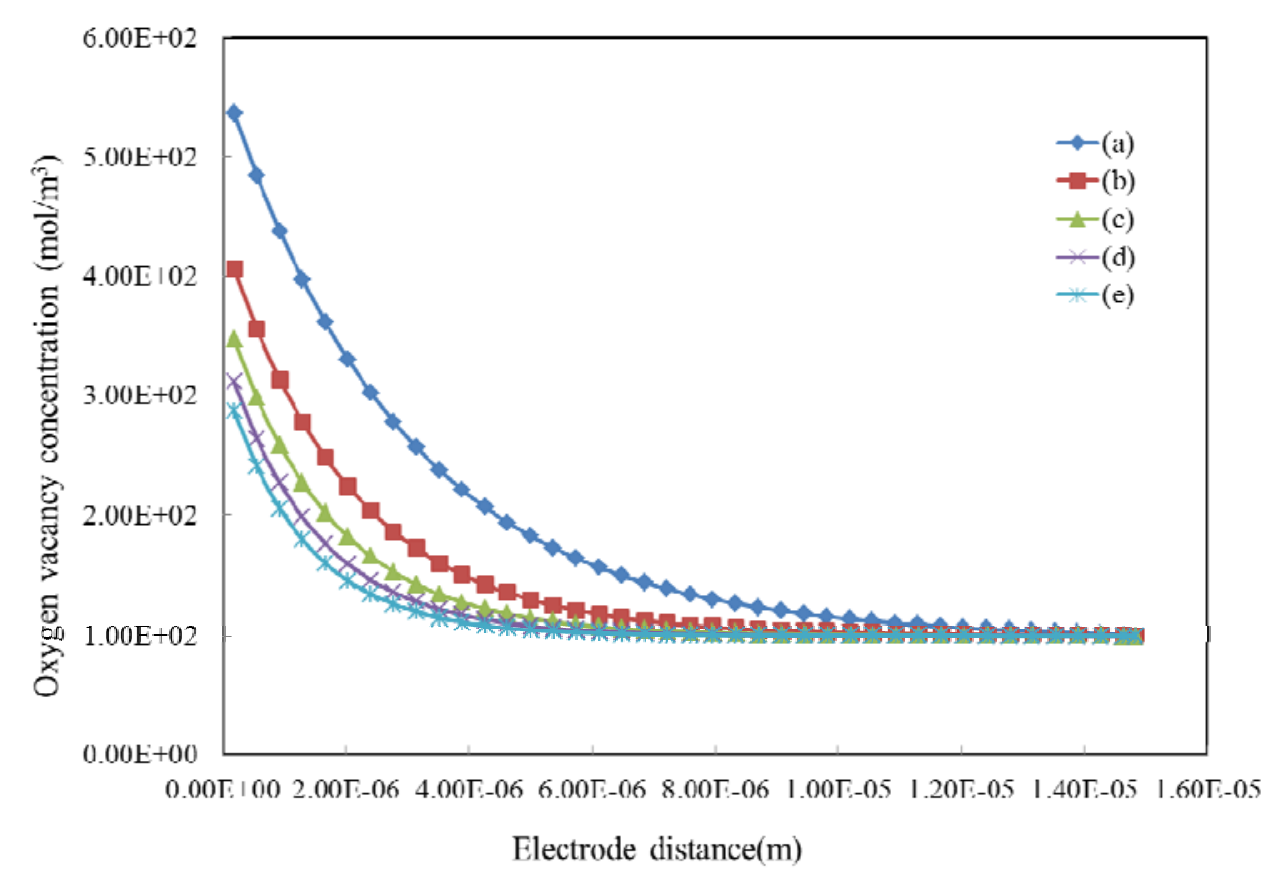

Figure 8.2 Oxygen concentration distributions in the cathode $(\eta=-0.2 \mathrm{~V})$ $r_{3,0}\left(\mathrm{~mol} / \mathrm{cm}^{2} \cdot \mathrm{s}\right)$ value is: (a) $1 \times 10^{-8}$ (b) $2 \times 10^{-8}$ (c) $3 \times 10^{-8}$ (d) $4 \times 10^{-8}$ (e) $5 \times 10^{-8}$ 


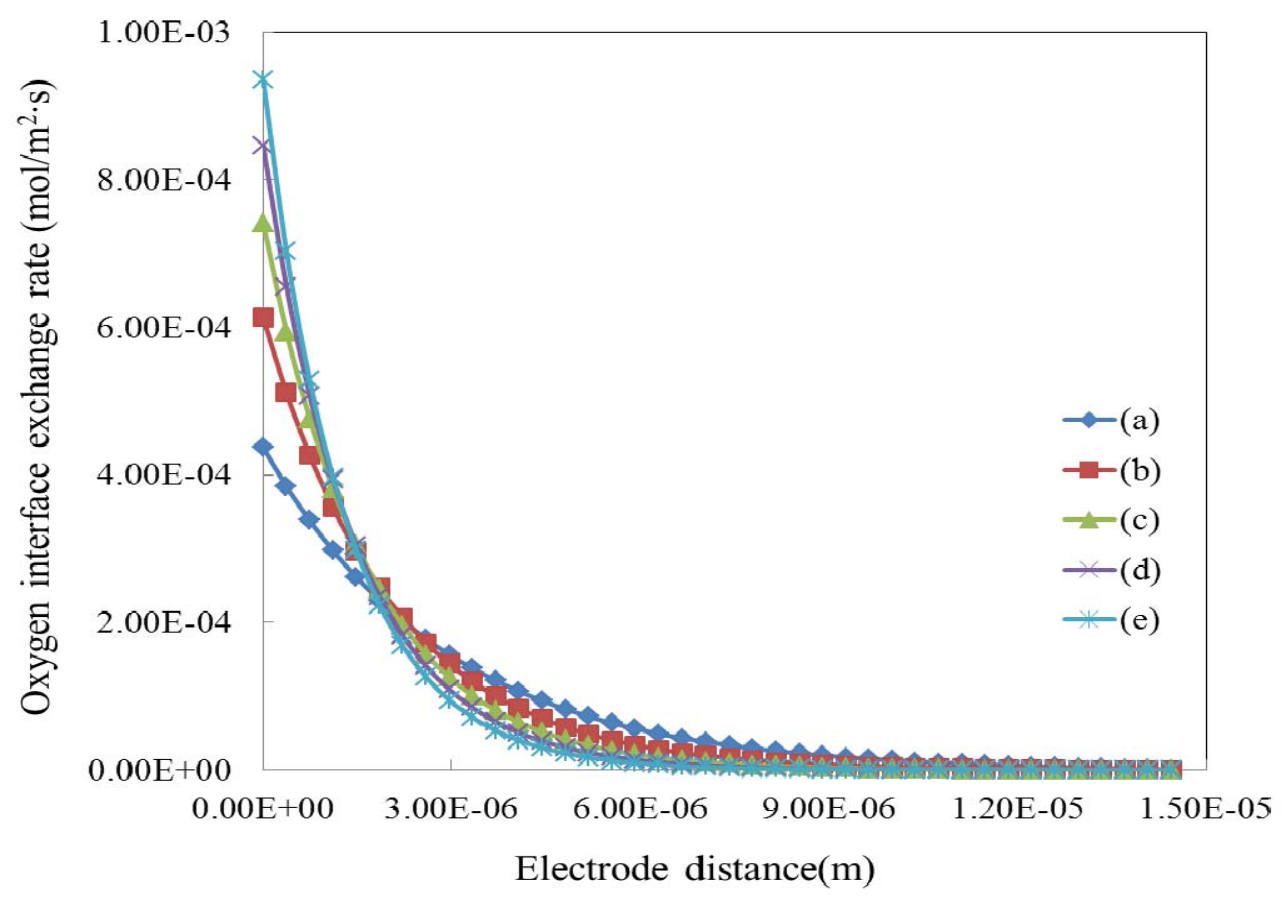

Figure 8.3 Oxygen interface exchange rate variation with the cathode thickness $(\eta=-0.2 \mathrm{~V})$ $r_{3,0}\left(\mathrm{~mol} / \mathrm{cm}^{2} \cdot \mathrm{s}\right)$ value is: (a) $1 \times 10^{-8}$ (b) $2 \times 10^{-8}$ (c) $3 \times 10^{-8}$ (d) $4 \times 10^{-8}$ (e) $5 \times 10^{-8}$

Figure 8.3 shows the corresponding simulation results for oxygen exchange rate at infiltrate/cathode backbone interface. The results also revealed that oxygen reaction active zone length decreased with increasing $r_{3,0}$ value. This conclusion indicated thin functional interlayer could be considered for infiltrated cathode. It is well know that the thickness of the interlayer can affect its porosity and the porosity is lower with a thinner interlayer. Low porosity of the functional interlayer could increase TPB length and effective electrical conductivities. Therefore, besides promoting the surface exchange rate, the infiltrated cathode also supports optimization of the cathode structure. Moreover, with the specific oxygen transport kinetic parameters selected, the advantage of the high $r_{3,0}$ value infiltrated material disappeared after $x=2 \mu \mathrm{m}$. 


\subsubsection{Over-potential effects}

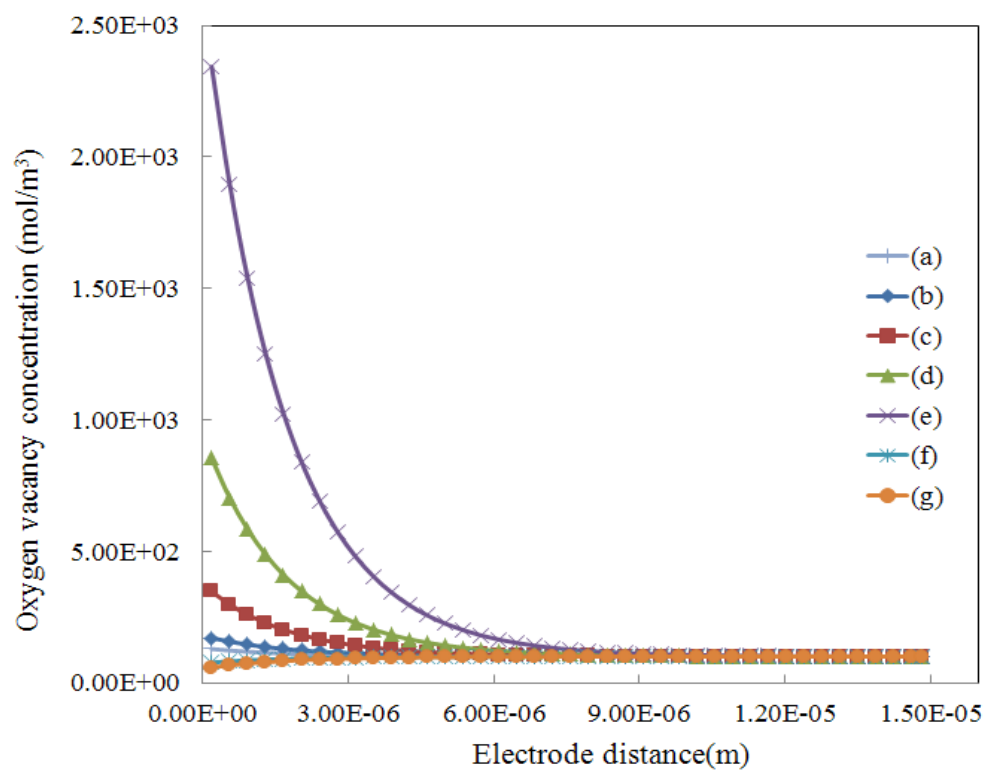

Figure 8.4 Oxygen vacancy concentration distributions in the cathode $\left(r_{3,0}=3 \times 10^{-8} \mathrm{~mol} / \mathrm{cm}^{2} \cdot \mathrm{s}\right)$

Over-potential: (a) -0.05V (b) -0.1V (c) $-0.2 \mathrm{~V}$ (d) $-0.3 \mathrm{~V}$ (e) $-0.4 \mathrm{~V}$ (f) $0.05 \mathrm{~V}$ (g) $0.1 \mathrm{~V}$

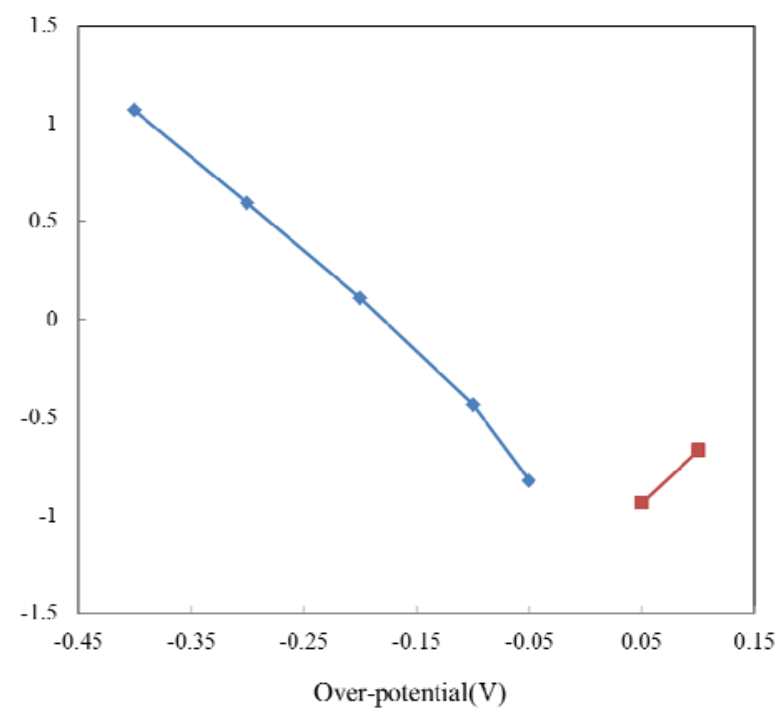

Figure 8.5 Logarithmic 2PB current density versus over-potential with $r_{3,0}=3 \times 10^{-8} \mathrm{~mol} / \mathrm{cm}^{2} \cdot \mathrm{s}$ 
The oxygen vacancy concentration distribution along the cathode under different over-potentials with the same $r_{3,0}$ value is presented in Figure 8.4. And Figure 8.5 shows over-potential effects on 2PB current density. Since mainly negative over-potential was built up at the cathode under SOFCs operation condition, no more anodic polarization beyond $0.1 \mathrm{~V}$ has been simulated. When the over-potential is higher than $0.1 \mathrm{~V}, 2 \mathrm{~PB}$ current follows the Tafel equation. Exchange current density can be obtained by calculating the Tafel slope and its result is $0.14 \mathrm{~A} / \mathrm{cm}^{2}$.

\subsection{Concluding remarks}

In this chapter, a mathematical one-dimensional model was developed for an infiltrated SOFC cathode. Oxygen transport at the infiltrate/cathode backbone interface was the research focus and was considered as the main pathway. The oxygen interface exchange rate value was estimated from ECR measurement results in Chap 7.

Simulation results indicated that no obvious variation of $2 \mathrm{~PB}$ current density (current density at cathode/electrolyte interface) was detected with different oxygen exchange rate at infiltrate/cathode backbone material interface. However, the characteristic length of the cathode decreased with the increasing interface oxygen ion exchange rate. It benefits optimization of cathode structure. 


\section{Conclusions and Recommendations}

\subsection{Introduction}

In last decades, tremendous efforts have been devoted to modeling the SOFC cathode reaction process. However, due to the fact that the reaction system and conditions are very complex, a lot of problems and debates still exist in this field so far. In this chapter, we will summarize some of the general conclusions emerging from this thesis and also highlight areas where future work is likely to make an impact.

\subsection{Conclusions}

The main objective of this research is to understand oxygen transport behavior in SOFCs cathode utilizing electrical conductivity relaxation $(E C R)$ technique. We first carefully discuss the ECR data fitting process in Chapter 5. The results showed that the fitted results varied with initial values for analyzing single relaxation data set. An improved method of testing two sample thicknesses under the same conditions was developed to fix oxygen surface exchange and bulk diffusion coefficients simultaneously.

With the improved data fitting method, we observed a widely used cathode material $\mathrm{La}_{0.6} \mathrm{Sr}_{0.4} \mathrm{Co}_{0.2} \mathrm{Fe}_{0.8} \mathrm{O}_{3-\delta}$ in Chapter 6. The relaxation process and oxygen contributions have been discussed under three control regions, including surface exchange, bulk diffusion and mixed control region. Relationships among oxygen surface exchange coefficient, oxygen partial pressure and the reduction reaction constants were deduced based on the discussion. This is a key achievement for combining ECR measuring results with the cathode modeling. 
Chapter 7 presents results for characterizing infiltrated cathode materials. Oxygen exchange coefficient at infiltrated/ cathode backbone interface was obtained by modifying the traditional ECR diffusion model. Two infiltrated materials were studied and the results showed that under high oxygen partial pressure $\mathrm{Ce}_{0.8} \mathrm{Sm}_{0.2} \mathrm{O}_{1.9}$ improve oxygen transport in LSCF cathode more than $\mathrm{La}_{0.6} \mathrm{Sr}_{0.4} \mathrm{CoO}_{3-\delta}$.

Finally, the infiltrated cathode mechanism was explored. We focused on the 2PB oxygen transport pathway to study the function of the infiltrated material. Given by the experimental results, oxygen transport at the interface of infiltrate/cathode backbone was considered as the rate limiting step. The exchange current value is proportional to $P_{O_{2}}^{-1 / 2}$. The oxygen vacancy distribution at steady state with low over-potential was also simulated.

\subsection{Recommendations for future research}

\subsubsection{ECR testing condition improvement}

9.3.1.1 Oxygen partial pressure broaden

Oxygen partial pressure testing range for our present ECR testing system is from $0.002 \mathrm{~atm}$ to $0.20 \mathrm{~atm}$. Broadening of the $P_{\mathrm{O}_{2}}$ testing range can not only improve understanding of the materials we observed but can also facilitate study of different material types.

First, if we assume cathode over-potential satisfies Nernst equation:

$$
\eta=\frac{R T}{4 F} \ln \frac{P_{O 2}^{s}}{P_{O 2}^{I}}
$$


Where $\eta$ is the cathode over-potential, $P_{O 2}^{s}$ and $P_{O 2}^{I}$ are the oxygen partial pressure for cathode surface and cathode/electrolyte interface. If we use air for the cathode side, $P_{O 2}^{I}$ should be $2.64 \times 10^{-3} \mathrm{~atm}$ when the over-potential is $0.1 \mathrm{~V}$. Therefore, broadening the range of examined oxygen partial pressure could help us understand oxygen transport behavior more thoroughly.

Second, there are two main perovskite materials widely used as SOFCs cathode. One is $\mathrm{Ln}_{1-}$ ${ }_{x} \mathrm{Sr}_{\mathrm{x}} \mathrm{Co}_{1-\mathrm{y}} \mathrm{Fe}_{\mathrm{y}} \mathrm{O}_{3-\delta}$ with $\mathrm{Ln}=\mathrm{La}, \mathrm{Sm}, \mathrm{Ba}, \mathrm{Nd}, \mathrm{Gd}$, Dy. The other one is $\operatorname{Ln}_{1-\mathrm{x}} \mathrm{A}_{\mathrm{x}} \mathrm{M}_{1-\mathrm{y}} \mathrm{Mn}_{\mathrm{y}} \mathrm{O}_{3-\delta}$ with $\mathrm{Ln}=\mathrm{La}, \mathrm{Nd}, \mathrm{Pr} ; \mathrm{A}=\mathrm{Ca}, \mathrm{Sr}$ and $\mathrm{M}=$ transition metal. In contrast to the first type, the second type perovskite has been shown to be very poor oxygen ion conductivity but their electronic conductivity is sufficiently high as to make them an attractive cathode material. Electrical conductivity variation of $\mathrm{La}_{1-\mathrm{x}} \mathrm{Sr}_{\mathrm{x}} \mathrm{MnO}_{3-\delta}$ with oxygen partial pressure can be found in reference, as shown in Figure 9.1. Conductivity relaxation occurred when oxygen partial pressure is smaller than $10^{-10}$ atm. 


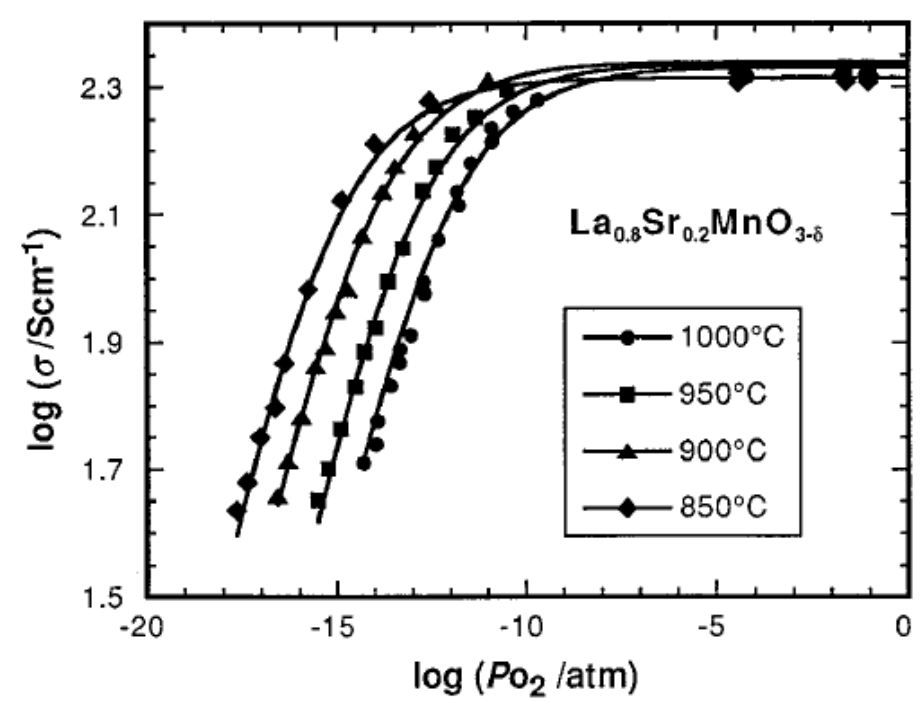

Figure 9.1 Electrical conductivity of LSM as a function of oxygen partial pressure at different temperature ${ }^{64}$

\subsubsection{Sample dimension}

As illustrated in Chapter 5, enlarging the samples' thickness difference can improve the accuracy for oxygen transport kinetic parameter estimation. However, for applying van der Pauw method, the sample's thickness was limited by its diameter. To further narrow the fitted $D$ and $k$ range, bar shape sample could be considered in the future research. The corresponding diffusion solution is:

$$
\frac{C(t)-C(0)}{C(\infty)-C(0)}=1-\sum_{n=1}^{\infty} \frac{4 L^{2} \exp \left[-b_{n}^{2} D t / a^{2}\right]}{b_{n}^{2}\left(b_{n}^{2}+L^{2}\right)}
$$

$b_{n}$ 's are the roots of

$$
b J_{1}(b)-L J_{0}(b)=0
$$

and

$$
L=k a / D
$$


a is the radius of the bar, [cm]. And as the solution for pellet shape sample, $L[-]$ is a unitless parameter and $b_{n}$ is a parameter generated during the process solving Fick's second law. $J_{0}$ and $J_{l}$ are the Bessel functions of zero and first order.

\subsubsection{Infiltrate/cathode backbone interface investigation}

\subsubsection{Isotope exchange technique verification}

Oxygen transport behavior can also be investigated by using labeled gases and secondary ion mass spectroscopy (SIMS). By utilizing SIMS technique, we can obtain oxygen self-diffusivity $\left(D^{*}\right)$ and exchange coefficient $\left(k^{*}\right)$ instead of chemical diffusivity and surface exchange coefficient. To further prove the reliability of the results obtained from ECR testing, SIMS (ims5f, CAMECA Instruments Co.) was also applied in this study. The corresponding oxygen partial pressure is 0.1 bar and the temperature is $873 \mathrm{~K}$ and $1073 \mathrm{~K}$.

The collected depth profile for ${ }^{18} \mathrm{O}$ was shown in Figure 9.2. The relation between selfdiffusivity and chemical diffusion coefficient is given by:

$$
D=D^{*} \Gamma
$$

Similar for the surface exchange coefficient:

$$
k=k^{*} \Gamma
$$

$\Gamma$ is the thermodynamic factor, [-]. $\Gamma$ is equal to 148 at $800^{\circ} \mathrm{C}$ and $P_{O_{2}}=0.1 \mathrm{~atm}^{66}$. Therefore, the isotope testing results can be transformed to chemical diffusivity and surface exchange coefficient and the corresponding values are $\mathrm{D}=5.2 \times 10^{-5} \mathrm{~cm}^{2} / \mathrm{s}, \mathrm{k}=1.5 \times 10^{-4} \mathrm{~cm} / \mathrm{s}$ and $\mathrm{D}=2.5 \times 10^{-}$ ${ }^{5} \mathrm{~cm}^{2} / \mathrm{s}, \mathrm{k}=4.1 \times 10^{-4} \mathrm{~cm} / \mathrm{s}$. On the other hand, the ECR testing results revealed at $800^{\circ} \mathrm{C}$ for LSCF 
$\mathrm{D}=5 \times 10^{-6} \mathrm{~cm}^{2} / \mathrm{s}$ and $\mathrm{k}=1 \times 10^{-4} \mathrm{~cm} / \mathrm{s}$. Considering the error range of isotope method, the parameter values obtained by utilizing the two techniques are approximately at the same magnitude. It's further verified that our analyzing results from ECR testing are reliable.

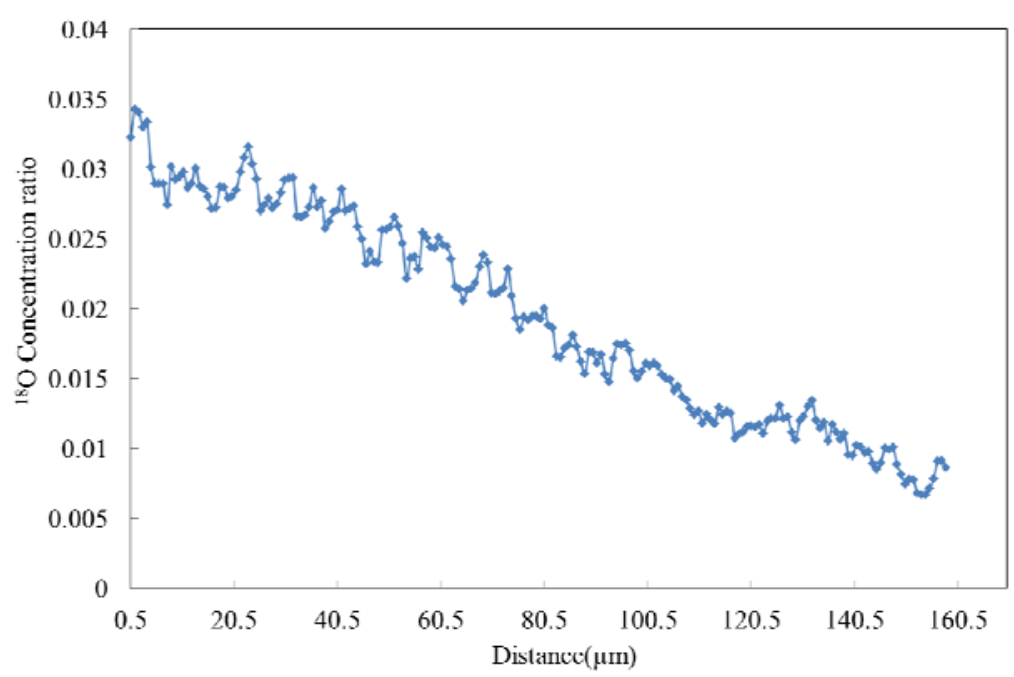

Figure 9.2 Depth profile of ${ }^{18} \mathrm{O}$ in $\mathrm{La}_{0.6} \mathrm{Sr}_{0.4} \mathrm{Co}_{0.2} \mathrm{Fe}_{0.8} \mathrm{O}_{3-\delta}$ at $800^{\circ} \mathrm{C}$

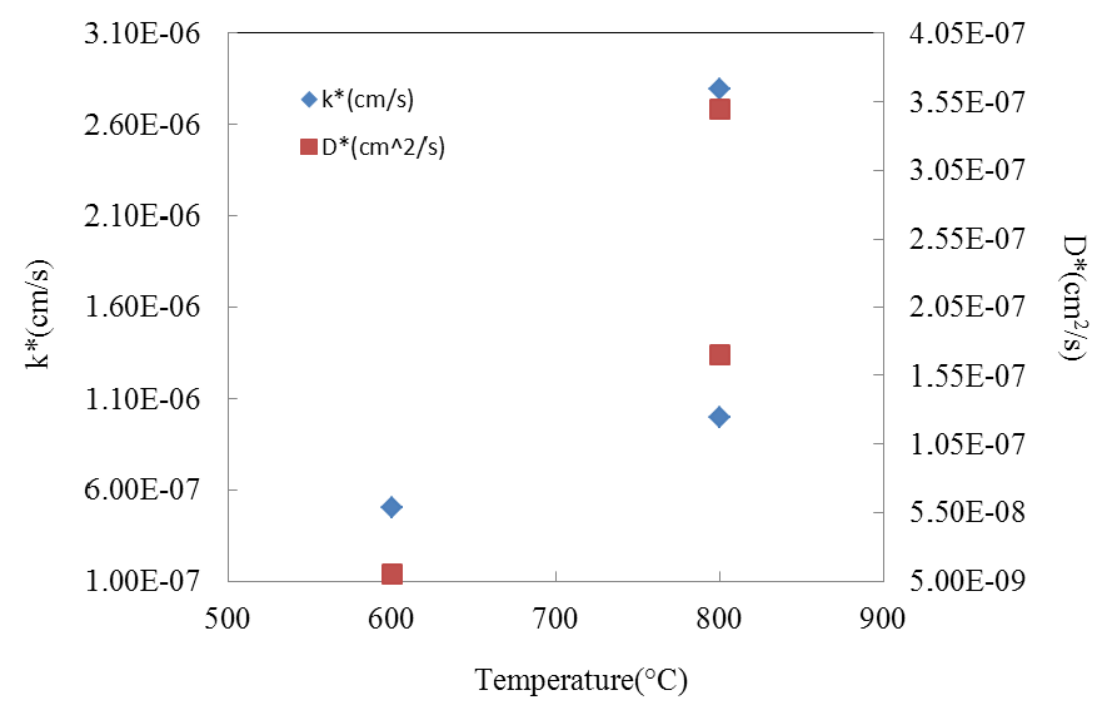

Figure 9.3 Self-surface exchange and bulk diffusivity of $\mathrm{La}_{0.6} \mathrm{Sr}_{0.4} \mathrm{Co}_{0.2} \mathrm{Fe}_{0.8} \mathrm{O}_{3-\delta}$ 
9.3.2.2 Further investigation for infiltrate/cathode backbone interface

In this study, oxygen transport behavior in an infiltrated cathode was characterized by utilizing the ECR technique. Characterization of micro-domains near the infiltrate/cathode backbone interface provides important information for analyzing the impact factors on oxygen diffusion.

With capability of imaging at a significantly higher resolution than SEM, transmission electron microscopy (TEM) can be applied to identity the phase near the hetero-interface. A TEM sample could be prepared by wedge polishing or focused ion etching. As a TEM measurement technique, electro energy loss spectroscopy provides elemental identification down to atomic dimensions and additional capabilities of determining electronic structure as well as chemical bonding. Ricoult $^{86}$ studied the interface of LSM-YSZ composite cathode by high spatial resolution TEM/EELS. After single cell operation, strong enrichment of divalent manganese was detected at LSM/YSZ interface. It was considered as a positive contribution for oxygen incorporation in cathode.

Besides the alteration of the defect chemistry, another main mechanism may contribute to effect on the oxygen ions transport at the hetero-interface. A favorable strain state at the interface can shift and/or change the symmetry of electron energy levels to provide for improved charge transfer and ion mobility. Yildiz ${ }^{87}$ et al. used density functional theory and the nudged elastic band method to compute oxygen vacancy migration paths and barriers in YZS as a function of lattice strain. 


\subsubsection{Infiltrated model development}

Oxygen transport in the infiltrated cathode is considered through the $2 \mathrm{~PB}$ pathway and composed of four elementary steps. Although the infiltrated model in this study was simulated under fair assumptions and with experimentally obtained parameters, the following aspects are suggested for future study:

(1) 3PB pathways:

To focus on the infiltrate/cathode backbone interface, the 3PB pathway was neglected in this study. Considering the 3PB pathway including infiltrate/cathode backbone/atmosphere and cathode backbone/electrolyte/atmosphere phase boundaries can complete our infiltrated model. Pattern electrode is one choice to investigate oxygen transport at the 3PB region.

(2) Surface and bulk over-potential of cathode backbone:

Considering the 3PB transport pathway, when electrons are brought to surface to form oxygen ions, positive charges are induced in cathode backbone and a potential barrier exits at the interface. Moreover, this potential barrier will be influenced by the cathode over-potential and the rate of oxygen ion diffusion will also be affected.

(3) 3-D model:

Cathode geometry and infiltrated material distribution had been ignored in our study too. After improved the model with the two items above, we can extend the model to three dimensions. 


\section{References}

${ }^{1}$ Mark C. Williams, Joseph P. Strakey, Wayne A. Surdoval. The U.S. Department of Energy, Office of Fossil Energy Stationary Fuel Cell Program. Journal of Power Sources, 2005; 143(1-2): 191-196.

${ }^{2}$ Zhongliang Zhan, Scott A. Barnett. An octane-Fueled Solid Oxide Fuel Cell. Science 2005; 308: 844-847.

${ }^{3}$ H. Zhao, F. Mauvy, C. Lalanne, J.-M. Bassat, S. Fourcade, J.-C. Grenier. New cathode materials for ITSOFC: Phase stability, oxygen exchange and cathode properties of $\mathrm{La}_{2-x} \mathrm{NiO}_{4+\delta}$. Solid State Ionics, 2008; 179(35-36): $2000-2005$.

${ }^{4}$ A. Princivalle, E. Djurado, Solid State Ionics, 2008; 179 (33-34): 1921-1928.

${ }^{5}$ Nguyen Q. Minh. Solid oxide fuel cell technology—features and applications. Solid State Ionics, 2004; 174(1-4): $271-277$.

${ }^{6}$ Xiongwen Zhang, S.H. Chan, Guojun Li, H.K. Ho, Jun Li, Zhenping Feng. A review of integration strategies for solid oxide fuel cells. Journal of Power Sources, 2010; 195(3): 685-702.

${ }^{7}$ A. Boudghene Stambouli, E. Traversa. Solid oxide fuel cells (SOFCs): a review of an environmentally clean and efficient source of energy. Renewable and Sustainable Energy Reviews, 2002; 6(5): 433-455.

${ }^{8}$ Anchasa Pramuanjaroenkij, Sadik Kakaç, Xiang Yang Zhou. Mathematical analysis of planar solid oxide fuel cells International Journal of Hydrogen Energy, 2008; 33(10): 2547-2565.

${ }^{9}$ EG\&G Technical Services, Inc. Fuel Cell Handbook, Chap7,Page 5, Seventh Edition, 2004.

${ }^{10}$ Frank Tietz, Andreas Mai, Detlev Stöver. From powder properties to fuel cell performance - A holistic approach for SOFC cathode development. Solid State Ionics, 2008; 179(27-32): 1509-1515.

${ }^{11}$ Junichiro Mizusaki, Naoya Mori, Hiroshi Takai, Yuki Yonemura, Hidehiro Minamiue, Hiroaki Tagawa, Masayuki Dokiya, Hideaki Inaba, Kazunori Naraya, Tadashi Sasamoto, Takuya Hashimoto. Oxygen nonstoichiometry and defect equilibrium in the perovskite-type oxides $\mathrm{La}_{1-\mathrm{x}} \mathrm{Sr}_{\mathrm{x}} \mathrm{MnO}_{3+\mathrm{d}}$. Solid State Ionics, 2000; 129(1-4): $163-177$.

${ }^{12}$ Tsepin Tsai, Scott A. Barnett. Effect of LSM-YSZ cathode on thin-electrolyte solid oxide fuel cell performance. Solid State Ionics, 1997; Volume 93(3-4): 207-217.

${ }^{13}$ R. A. De Souza, J. A. Kilner, J. F. Walker. A SIMS study of oxygen tracer diffusion and surface exchange in $\mathrm{La}_{0.8} \mathrm{Sr}_{0.2} \mathrm{MnO}_{3+\delta}$. Materials Letters, 2000; 43(1-2): 43-52. 
${ }^{14}$ P. Leone, M. Santarelli, P. Asinari, M. Calì, R. Borchiellini. Experimental investigations of the microscopic features and polarization limiting factors of planar SOFCs with LSM and LSCF cathodes. Journal of Power Sources 2008; 177(1): 111-122.

${ }^{15}$ Vladimir V. Srdić, Radovan P. Omorjan, Johannes Seydel. Electrochemical performances of (La,Sr)CoO $\mathrm{Cathode}_{3}$ for zirconia-based solid oxide fuel cells. Materials Science and Engineering B, 2005; 116(2):119-124.

${ }^{16}$ Changrong Xia, William Rauch, Fanglin Chen, Meilin Liu. $\mathrm{Sm}_{0.5} \mathrm{Sr}_{0.5} \mathrm{CoO}_{3}$ cathodes for low-temperature SOFCs. Solid State Ionics, 2002; 149(1-2): 11-19.

${ }^{17}$ H. Fukunaga, M. Koyama, N. Takahashi, C. Wen, K. Yamada. Reaction model of dense $\mathrm{Sm}_{0.5} \mathrm{Sr}_{0.5} \mathrm{CoO}_{3}$ as $\mathrm{SOFC}$ cathode. Solid State Ionics, 2000; 132(3-4): 279-285.

${ }^{18}$ Hong Lv, Yu-ji Wu, Bo Huang, Bin-yuan Zhao, Ke-ao Hu. Structure and electrochemical properties of $\mathrm{Sm}_{0.5} \mathrm{Sr}_{0.5} \mathrm{Co}_{1-\mathrm{x}} \mathrm{Fe}_{\mathrm{x}} \mathrm{O}_{3-\delta}$ cathodes for solid oxide fuel cells. Solid State Ionics, 2006; 177(9-10): 901-906.

19 Xifeng Ding, Chong Cui, Xuejuan Du, Lucun Guo. Electrical conductivity, thermal expansion and electrochemical properties of Fe-doped $\mathrm{La}_{0.7} \mathrm{Sr}_{0.3} \mathrm{CuO}_{3-\delta}$ cathodes for solid oxide fuel cells. Journal of Alloys and Compounds, 2009; 475(1-2): 418-421.

${ }^{20}$ Wei Zhou, Ran Ran, Zongping Shao. Progress in understanding and development of $\mathrm{Ba}_{0.5} \mathrm{Sr}_{0.5} \mathrm{Co}_{0.8} \mathrm{Fe}_{0.2} \mathrm{O}_{3-\delta}$-based cathodes for intermediate-temperature solid-oxide fuel cells: A review. Journal of Power Sources, 2009; 192(2): $231-246$.

${ }^{21}$ S. B. Adler, J. A. Lane, B. C. H. Steele. Electrode Kinetics of Porous Mixed-Conducting Oxygen Electrodes. J. Electrochem. Soc., 1996; 143(11): 3554-3564.

${ }^{22}$ M. Liu, J. Winnick. Comments for Electrode kinetics of porous mixed-conducting oxygen electrodes. J. Electrochem. Soc. Vol., 1997; 144(5): 1881-1883.

23 S. B. Adler. Limitations of charge-transfer models for mixed-conducting oxygen electrodes. Solid State Ionics, 2000;135(1-4): 603-612.

${ }^{24}$ Ann Mari Svensson, Svein Sunde, Kemal Nisancioglu. A mathematical model of the porous SOFC cathode. Solid State Ionics, 1996; 86-88(2): 1211-1216. 
${ }^{25}$ Ann Mari Svensson, Svein Sunde, Kemal Nisancioglu. Mathematical Modeling of Oxygen Exchange and Transport in Air-Perovskite-YSZ Interface Regions I. Reduction of Intermediately Adsorbed Oxygen. J. Electrochem. Soc., 1997; 144(8): 2719-2732.

${ }^{26}$ Gregory W. Coffey, Larry R. Pederson, and Peter C. Rieke. Competition Between Bulk and Surface Pathways in Mixed Ionic Electronic Conducting Oxygen Electrodes. Journal of The Electrochemical Society, 2003; 150(8): $1139-1151$.

${ }^{27}$ George Kopasakis, Thomas Brinson and Sydni Credle, Ming Xu.A Theoretical Solid Oxide Fuel Cell Model for System Controls and Stability Design. NASA/TM-2006-214104.

${ }^{28}$ Meilin Liu, Jack Winnick. Fundamental issues in modeling of mixed ionic-electronic conductors (MIECs). Solid State Ionics, 1999; 118(1-2): 11-21.

${ }^{29}$ F. H. van Heuveln, H. J. M. Bouwmeester. Electrode Properties of Sr-Doped $\mathrm{LaMnO}_{3}$ on Yttria-Stabilized Zirconia II. Elecfrocle Kinetics. J. Electrochem. Soc., 1997; 144(1):135-140.

${ }^{30}$ Mitterdorfer A. Identification of the Oxygen Reduction at Cathodes of Solid Oxide Fuel Cells, Ph.D. Dissertation. Swiss Federal Institute of Technology, 1997.

${ }^{31}$ S. H. Chan, X. J. Chen, K. A. Khor. Cathode Micro model of Solid Oxide Fuel Cell. Journal of The Electrochemical Society, 2004; 151(1): A164-A172.

${ }^{32}$ Bilge Yildiz, Gerardo Jose la O', Yang Shao-Horn. Analytical and experimental study of oxygen reduction mechanism on oxygen electrodes of SOFCs: Case study on $\mathrm{La}_{(1-\mathrm{x})} \mathrm{Sr}_{\mathrm{x}} \mathrm{MnO}_{3}$. Prepr. Pap.-Am. Chem. Soc., Div. Fuel Chem. 2004, 49 (2): 757-758.

33 Jeng-Han Wang, MeiLin Liu, M.C. Lin. Oxygen reduction reactions in the SOFC cathode of $\mathrm{Ag} / \mathrm{CeO}_{2}$ Solid State Ionics, 2006; 177(9-10): 939-947.

${ }^{34}$ Jurgen Fleig. On the current-voltage characteristics of charge transfer reactions at mixed conducting electrodes on solid electrolytes. Phys. Chem. Chem. Phys., 2005; 7: 2027-2037.

${ }^{35}$ F.S. Baumann, J. Maier, J. Fleig. The polarization resistance of mixed conducting SOFC cathodes: A comparative study using thin film model electrodes. Solid State Ionics, 2008;179(21-26): 1198-1204.

${ }^{36}$ Frank S. Baumann, Jürgen Fleig, Hanns-Ulrich Habermeier, Joachim Maier. Impedance spectroscopic study on well-defined $(\mathrm{La}, \mathrm{Sr})(\mathrm{Co}, \mathrm{Fe}) \mathrm{O}_{3-\delta}$ model electrodes. Solid State Ionics, 2006; 177(11-12): 1071-1081. 
${ }^{37}$ David S. Mebane, Yingjie Liu, Meilin Liu. A Two-Dimensional Model and Numerical Treatment for Mixed Conducting Thin Films. Journal of The Electrochemical Society, 2007; 154(5): A421-A426.

${ }^{38}$ David S. Mebane, Meilin Liu. Classical, phenomenological analysis of the kinetics of reactions at the gas-exposed surface of mixed ionic electronic conductors. J Solid State Electrochem, 2006; 10: 575-580.

${ }^{39}$ M. Sahibzada, S. J. Benson, R. A. Rudkin, J. A. Kilner. Pd-promoted $\mathrm{La}_{0.6} \mathrm{Sr}_{0.4} \mathrm{Co}_{0.2} \mathrm{Fe}_{0.8} \mathrm{O}_{3}$ cathodes. Solid State Ionics, 1998; 113-115: 285-290.

${ }^{40}$ Jiang S P, Wang W. Novel structured mixed ionic and electronic conducting cathodes of solid oxide fuel cells. Solid State Ionics, 2005, 176: 1351-1357.

${ }^{41}$ Xia C, Xu X, Jiang Z, Fan X. LSM-SDC electrodes fabricated with an ion-impregnating process for SOFCs with doped ceria electrolytes. Solid State Ionics, 2006, 177 (19-25): 2113-2117.

${ }^{42}$ Wang S, Kato T, Nagata S, Honda T, Kaneko T, Iwashita N, Dokiya M. Performance of a $\mathrm{La}_{0.6} \mathrm{Sr}_{0.4} \mathrm{Co}_{0.8} \mathrm{Fe}_{0.2} \mathrm{O}_{3^{-}}$ $\mathrm{Ce}_{0.8} \mathrm{Gd}_{0.2} \mathrm{O}_{1.9}$-Ag cathode for ceria electrolyte SOFCs. Solid State Ionics, 2002, 146(3-4): 203-210.

${ }^{43}$ Sholklapper T Z, Radmilovic V, Jacobson C P, Visco S J, De Jonghe L C. Nanocomposite Ag-LSM solid oxide fuel cell electrodes. Journal of Power Sources, 2008, 175(1): 206-210.

${ }^{44}$ Yamahara K, Jacobson C P, Visco S J, De Jonghe L C. Catalyst-infiltrated supporting cathode for thin-film SOFCs. Solid State Ionics, 2005, 176(5-6): 451-456.

${ }^{45}$ Jiang Z Y, Zhang L, Feng K, Xia C R. Nanoscale bismuth oxide impregnated (La,Sr)MnO3 cathodes for intermediate-temperature solid oxide fuel cells. Journal of Power Sources, 2008, 185(1): 40-48.

${ }^{46}$ Yingyi Huang, John M. Vohs, Raymond J. Gorte. An Examination of LSM-LSCo Mixtures for Use in SOFC Cathodes. Journal of The Electrochemical Society, 2006, 153(6): A951-A955.

${ }^{47}$ Lin Xiong, Shaorong Wang, Zhenrong Wang, Tinglian Wen. Effect of samarium doped ceria nanoparticles impregnation on the performance of anode supported SOFC with $\left(\operatorname{Pr}_{0.7} \mathrm{Ca}_{0.3}\right)_{0.9} \mathrm{MnO}_{3-\delta}$ cathode. Journal of Rare Earths, 2010; 28(1): 96-99.

${ }^{48}$ Shiwoo Lee, Nicholas Miller, Harry Abernathy, Kirk Gerdes, and Ayyakkannu Manivannan. Effect of Sr-Doped $\mathrm{LaCoO}_{3}$ and $\mathrm{LaZrO}_{3}$ Infiltration on the Performance of SDC-LSCF Cathode. J. Electrochem. Soc., 2011;158: B735B742. 
${ }^{49}$ Chang-Rock Song, Han-Ill Yoo. Chemical diffusivity of $\mathrm{BaTiO}_{3-\delta}$ : I. Experimental determination. Solid State Ionics, 1999; 120(1-4): 141-153.

${ }^{50}$ J. Crank. The mathematics of diffusion, Clarendon Press, Oxford, UK, second edition, 1975, page60.

${ }^{51}$ H.J.M. Bouwmeester, H. Kruidhof, A.J. Burggraaf . Importance of the surface exchange kinetics as rate limiting step in oxygen permeation through mixed-conducting oxides. Solid State Ionics, 1994, 72(2): 185-194.

${ }^{52}$ R. J. Chater, S. Carter, J. A. Kilner, B. C. H. Steele. Development of a novel SIMS technique for oxygen selfdiffusion and surface exchange coefficient measurements in oxides of high diffusivity. Solid State Ionics, 1992; 5356(2): $859-867$.

${ }^{53}$ N. Sakai, K. Yamaji, T. Horita, H. Kishimoto, M.E. Brito, H. Yokokawa, Y. Uchimoto. Application of SIMS analyses on oxygen transport in SOFC materials. Applied Surface Science, 2006; 252(19): 7045-7047.

${ }^{54}$ R. A. De Souza, J. A. Kilner. Oxygen transport in $\mathrm{La}_{1-\mathrm{x}} \mathrm{Sr}_{\mathrm{x}} \mathrm{Mn}_{1-\mathrm{y}} \mathrm{Co}_{\mathrm{y}} \mathrm{O}_{3 \pm \delta}$ perovskites: Part I. Oxygen tracer diffusion. Solid State Ionics, 1998; 106(3-4): 175-187.

${ }^{55}$ R. A. De Souza, J. A. Kilner. Oxygen transport in $\mathrm{La}_{1-\mathrm{x}} \mathrm{Sr}_{\mathrm{x}} \mathrm{Mn}_{1-\mathrm{y}} \mathrm{Co}_{\mathrm{y}} \mathrm{O}_{3 \pm \delta}$ perovskites: Part II. Oxygen surface exchange. Solid State Ionics, 1999; 126(1-2): 153-161.

${ }^{56}$ J. A. Kilner, R. A. De Souza, I. C. Fullarton. Surface exchange of oxygen in mixed conducting perovskite oxides. Solid State Ionics, 1996; 86-88(2): 703-709.

${ }^{57}$ Isamu Yasuda, Kei Ogasawara, Masakazu Hishinuma, Tatsuya Kawada, Masayuki Dokiya. Oxygen tracer diffusion coefficient of (La, Sr) $\mathrm{MnO}_{3 \pm \delta}$. Solid State Ionics, 1996; 86-88(2): 1197-1201.

${ }^{58}$ Edwin S. Raj, John A. Kilner, John T.S. Irvine. Oxygen diffusion and surface exchange studies on $\left(\mathrm{La}_{0.75} \mathrm{Sr}_{0.25}\right)_{0.95} \mathrm{Cr}_{0.5} \mathrm{Mn}_{0.5} \mathrm{O}_{3-\delta}$. Solid State Ionics, 2006; 177(19-25): 1747-1752.

${ }^{59}$ X. Chen, S. Wang, Y. L. Yang, L. Smith, N. J. Wu, B. -I. Kim, S. S. Perry, A. J. Jacobson, A. Ignatiev. Electrical conductivity relaxation studies of an epitaxial $\mathrm{La}_{0.5} \mathrm{Sr}_{0.5} \mathrm{CoO}_{3-\delta}$ thin film. Solid State Ionics, 2002; 146(3-4): 405-413. ${ }^{60}$ F. Mauvy, J. M. Bassat, E. Boehm, P. Dordor, J. C. Grenier, J. P. Loup. Chemical oxygen diffusion coefficient measurement by conductivity relaxation-correlation between tracer diffusion coefficient and chemical diffusion coefficient. Journal of the European Ceramic Society, 2004; 24(6): 1265-1269.

${ }^{61}$ G. Kim, S. Wang, A.J. Jacobson, C.L. Chen. Measurement of oxygen transport kinetics in epitaxial $\mathrm{La}_{2} \mathrm{NiO}_{4+\delta}$ thin films by electrical conductivity relaxation. Solid State Ionics, 2006; 177(17-18): 1461-1467. 
${ }^{62}$ W. Preis, E. Bucher, W. Sitte. Oxygen exchange kinetics of $\mathrm{La}_{0.4} \mathrm{Sr}_{0.6} \mathrm{FeO}_{3-\delta}$ by simultaneous application of conductivity relaxation and carrier gas coulometry. Solid State Ionics, 2004; 175(1-4): 393-397.

${ }^{63}$ Isamu Yasuda, Tomoji Hikita. Precise Determination of the Chemical Diffusion Coefficient of Calcium-Doped Lanthanum Chromites by Means of Electrical Conductivity Relaxation. J. Electrochern. Soc., 1994; 141(5): 12681273.

${ }^{64}$ Isamu Yasuda, Masakazu Hishinuma. Electrical Conductivity and Chemical Diffusion Coefficient of StrontiumDoped Lanthanum Manganites. Journal of Solid State Chemistry, 1996; 123(2): 382-390.

${ }^{65}$ J. A. Lane, J. A. Kilner. Measuring oxygen diffusion and oxygen surface exchange by conductivity relaxation. Solid State Ionics, 2000; 136-137(2): 997-1001.

${ }^{66}$ S.Wang, P.A.W. van der Heide, C. Chavez, A. J. Jacobson, S. B. Adler. An electrical conductivity relaxation study of $\mathrm{La}_{0.6} \mathrm{Sr}_{0.4} \mathrm{Fe}_{0.8} \mathrm{Co}_{0.2} \mathrm{O}_{3-\delta}$. Solid State Ionics, 2003; 156(1-2): 201-208.

${ }^{67}$ M. W. den Otter,z H. J. M. Bouwmeester, B. A. Boukamp, H. Verweij. Reactor Flush Time Correction in Relaxation Experiments. Journal of the Electrochemical Society, 2001; 148(2): J1-J6.

${ }^{68}$ S. Wang, A. Verma, Y. L. Yang, A. J. Jacobson, Ben Abeles. The effect of the magnitude of the oxygen partial pressure change in electrical conductivity relaxation measurements: oxygen transport kinetics in $\mathrm{La}_{0.5} \mathrm{Sr}_{0.5} \mathrm{CoO}_{3-\delta}$. Solid State Ionics, 2001; 140(1-2): 125-133.

${ }^{69}$ Rosemary A. Cox-Galhotra, Steven McIntosh. Unreliability of simultaneously determining $\mathrm{k}_{\text {chem }}$ and $\mathrm{D}_{\text {chem }}$ via conductivity relaxation for surface-modified $\mathrm{La}_{0.6} \mathrm{Sr}_{0.4} \mathrm{Co}_{0.2} \mathrm{Fe}_{0.8} \mathrm{O}_{3-\delta}$. Solid State Ionics, 2010; 181(31-32): 14291436.

${ }^{70}$ M. W. den Otter,z H. J. M. Bouwmeester, B. A. Boukamp, H. Verweij. Reactor Flush Time Correction in Relaxation Experiments. Journal of the Electrochemical Society, 2001; 148(2): J1-J6.

${ }^{71}$ S. Wang, A. Verma, Y. L. Yang, A. J. Jacobson, Ben Abeles. The effect of the magnitude of the oxygen partial pressure change in electrical conductivity relaxation measurements: oxygen transport kinetics in $\mathrm{La}_{0.5} \mathrm{Sr}_{0.5} \mathrm{CoO}_{3-\delta}$. Solid State Ionics, 2001; 140(1-2): 125-133.

${ }^{72}$ Chang-Rock Song, Han-Ill Yoo. Chemical diffusivity of $\mathrm{BaTiO}_{3-\delta}$ : I. Experimental determination. Solid State Ionics, 1999;120(1-4): 141-153. 
${ }^{73}$ L. J. van der Pauw. A method of measuring specific resistivity and Hall effect of discs of arbitrary shape. Philips Research Reports, 1958; 13(1): 1-9.

${ }^{74}$ Dionissios Mantzavinos, Anne Hartley, Ian S Metcalfe, Mortaza Sahibzada. Oxygen stoichiometries in $\mathrm{La}_{1-\mathrm{x}} \mathrm{Sr}_{\mathrm{x}} \mathrm{Co}_{1-\mathrm{y}} \mathrm{Fe}_{\mathrm{y}} \mathrm{O}_{3-\delta}$ perovskites at reduced oxygen partial pressures. Solid State Ionics, 2000; 134(1-2): 103-109.

${ }^{75}$ J. A. Kilner, R. A. De Souza, I. C. Fullarton. Surface exchange of oxygen in mixed conducting perovskite oxides. Solid State Ionics, 1996; 86-88(2): 703-709.

${ }^{76}$ Junichiro Mizusaki, Isamu Yasuda, Jun-ichi Shimoyama, Shigeru Yamauchi, Kazuo Fueki. Electrical Conductivity, Defect Equilibrium and Oxygen Vacancy Diffusion Coefficient of $\mathrm{La}_{1-x} \mathrm{Ca}_{x} \mathrm{AlO}_{3-\delta}$ Single Crystals. J. Electrochem. Soc., 1993; 140(2): 467-471.

${ }^{77}$ Bjarke Thomas Dalslet, Martin Søgaard, Henny J.M. Bouwmeester, Peter Vang. Defect chemistry and oxygen transport of $\left(\mathrm{La}_{0.6} \mathrm{Sr}_{0.4-\mathrm{x}} \mathrm{M}_{\mathrm{x}}\right)_{0.99} \mathrm{Co}_{0.2} \mathrm{Fe}_{0.8} \mathrm{O}_{3-\delta}, \mathrm{M}=\mathrm{Ca}(\mathrm{x}=0.05,0.1), \mathrm{Ba}(\mathrm{x}=0.1,0.2)$, Sr: Part I: Defect chemistry. Solid State Ionics, 2009; 180(20-22): 1173-1182.

${ }^{78}$ R.H.E. van Doorn, A.J. Burggraaf. Structural aspects of the ionic conductivity of $\mathrm{La}_{1-\mathrm{x}} \mathrm{Sr}_{\mathrm{x}} \mathrm{CoO}_{3-\delta}$. Solid State Ionics, 2000; 128(1-4): 65-78.

${ }^{79}$ M. W. den Otter. A study of oxygen transport in mixed conducting oxides using isotopic exchange and conductivity relaxation. Thesis of the University of Enschede. 2000.

${ }^{80}$ Dong Ding, Mingyang Gong, Chunchuan Xu, et al. Electrochemical characteristics of samaria-doped ceria infiltrated strontium-doped $\mathrm{LaMnO}_{3}$ cathodes with varied thickness for yttria-stabilized zirconia electrolytes. Journal of Power Sources, 2011; 196(5): 2551-2557.

${ }^{81}$ Lifang Nie, Mingfei Liu, Yujun Zhang, Meilin Liu. $\mathrm{La}_{0.6} \mathrm{Sr}_{0.4} \mathrm{Co}_{0.2} \mathrm{Fe}_{0.8} \mathrm{O}_{3-\delta}$ cathodes infiltrated with samariumdoped cerium oxide for solid oxide fuel cells. Journal of Power Sources, 2010; 195(15): 4704-4708.

${ }^{82}$ Toshiaki Matsui, Minoru Inaba, Atsushi Mineshige, Zempachi Ogumi. Electrochemical properties of ceria-based oxides for use in intermediate-temperature SOFCs. Solid State Ionics, 2005; 176(7-8): 647-654.

${ }^{83}$ E. N. Armstrong, K. L. Duncan, D. J. Oh, J. F. Weaver, and E. D. Wachsman. Determination of Surface Exchange Coefficients of LSM, LSCF, YSZ, GDC Constituent Materials in Composite SOFC Cathodes. J. Electrochem. Soc., 2011; 158: B492-499.

${ }^{84}$ Ramanan Ganeshananthan, Anil V. Virkar. Measurement of Surface Exchange Coefficient on Porous 
$\mathrm{La}_{0.6} \mathrm{Sr}_{0.4} \mathrm{CoO}_{3-\delta}$ Samples by Conductivity Relaxation. Journal of The Electrochemical Society, 2005; 152(8): A1620-A1628.

${ }^{85}$ Mingyang Gong, Randall S. Gemmen, Xingbo Liu. Modeling of oxygen reduction mechanism for 3PB and 2PB pathways at solid oxide fuel cell cathode from multi-step charge transfer. Journal of Power Sources, 2012; 201:204218.

${ }^{86}$ M. Backhaus-Ricoult. Interface chemistry in LSM-YSZ composite SOFC cathodes. Solid State Ionics, 2006, 177(19-25): 2195-2200.

${ }^{87}$ Akihiro Kushima, Bilge Yildiz. Oxygen ion diffusicity in strained yttria stabilized zirconia: where is the fastest strain?. J. Mater. Chem., 2010; 20: 4809-4819. 


\section{Published papers}

Yihong Li, Kirk Gerdes, Harvey Diamond, Xingbo Liu. An improved method to increase the predictive accuracy of the ECR technique. Solid State Ionics, 2011;204-205: 104-110.

Yihong Li, Yinglu Jiang, Junwei Wu, Richard Pineault, Randall Gemmenn and Xingbo Liu. Corrosion behavior of Ebrite and SS430 in coal syngas with loaded current. International Journal of Applied Ceramic Technology, 2011; 8(1): 60-67.

Yihong Li, Randall Gemmen, Xingbo Liu. Oxygen reduction and Transportation Mechanisms in solid oxide fuel cell cathodes. Journal of Power Sources, 2010; 195(11): 3345-3358.

Yihong Li, Junwei Wu, Christopher Johnson, Randall Gemmen, X. Mao Scott, Xingbo Liu. Oxidation behavior of metallic interconnects for SOFC in coal syngas. International Journal of Hydrogen Energy, 2009; 34(3): 1489-1496.

Yihong Li, Yinglu Jiang, Junwei Wu, Richard Pineault, Randall Gemmenn and Xingbo Liu. Effects of Electrical Current on Solid Oxide Fuel Cells Metallic Interconnect Oxidation in Syngas. International Journal of Applied Ceramic Technology, 2009; 7(1):41-48.

\section{Conference presentations}

Yihong Li, Kirk Gerdes, Xingbo Liu. Oxygen transport investigation for SOFCs cathode. MS\&T' 11, Oct. 19, 2011.

Yihong Li, Kirk Gerdes, Xingbo Liu. Oxygen transport properties of $\mathrm{La}_{0.6} \mathrm{Sr}_{0.4} \mathrm{Co}_{0.2} \mathrm{Fe}_{0.8} \mathrm{O}_{3-\delta}$. ICACC' 11, Jan. 27, 2011.

Dong Ding, Yihong Li, Mingyang Gong, Xingbo Liu. SDC Infiltrated LSM Cathode for IntermediateTemperature SOFCs. MS\&T'10, Oct. 21, 2010.

$\underline{\mathbf{Y}}$ Li, K. Gerdes, R. Gemmen, X. Liu. Effect of the oxygen partial pressure change steps in electrical conductivity relaxation measurements. ICACC' 10, Jan. 26, 2010.

Yihong Li, Yinglv Jiang, Junwei Wu, Randall Gemmen, Xingbo Liu. Investigation of Current Effect on Ferritic Stainless Steels Corrosion Behavior in Coal Syngas. MS\&T’09, Oct. 27, 2009.

Y. Li, R. Ravi Dastane, Y. Jiang, X. Liu, C. Johnson, R. Gemmen. Oxidation behavior of Crofer 22 interconnects for SOFC in coal syngas. MS\&T'08, Oct. 8, 2008. 